\title{
Air Exchange Rate Impact on Actinon, Thoron, and Radon Activity Equilibrium Factor and Inhalation Fractional Equilibrium Factor Determination in Vapor Intrusion Risk and Dose Models
}

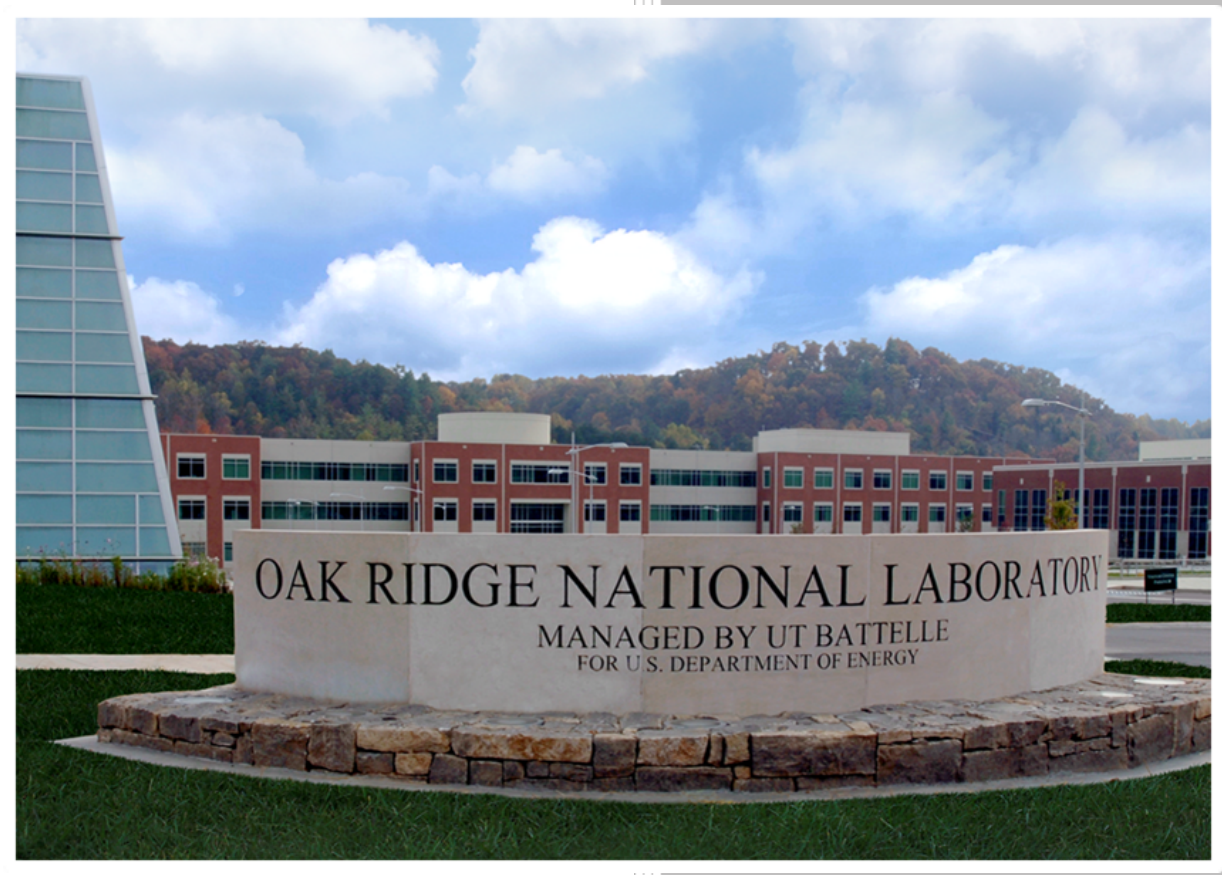

APPROVED FOR PUBLIC RELEASE. DISTRIBUTION IS UNLIMITED.
Ethan A. Asano Fredrick G. Dolislager Karessa L. Manning Debra J. Stewart Katie A. Noto Hayden J. Ringer Caleigh E. Samuels Leslie D. Galloway Anthony Q. Armstrong Michael B. Bellamy

September 2020 


\title{
DOCUMENT AVAILABILITY
}

Reports produced after January 1, 1996, are generally available free via US Department of Energy (DOE) SciTech Connect.

Website http://www.osti.gov/scitech/

Reports produced before January 1, 1996, may be purchased by members of the public from the following source:

\author{
National Technical Information Service \\ 5285 Port Royal Road \\ Springfield, VA 22161 \\ Telephone 703-605-6000 (1-800-553-6847) \\ TDD 703-487-4639 \\ Fax 703-605-6900 \\ E-mail info@ntis.gov \\ Website http://www.ntis.gov/help/ordermethods.aspx
}

Reports are available to DOE employees, DOE contractors, Energy Technology Data Exchange representatives, and International Nuclear Information System representatives from the following source:

Office of Scientific and Technical Information

PO Box 62

Oak Ridge, TN 37831

Telephone 865-576-8401

Fax 865-576-5728

E-mail reports@osti.gov

Website http://www.osti.gov/contact.html

This report was prepared as an account of work sponsored by an agency of the United States Government. Neither the United States Government nor any agency thereof, nor any of their employees, makes any warranty, express or implied, or assumes any legal liability or responsibility for the accuracy, completeness, or usefulness of any information, apparatus, product, or process disclosed, or represents that its use would not infringe privately owned rights. Reference herein to any specific commercial product, process, or service by trade name, trademark, manufacturer, or otherwise, does not necessarily constitute or imply its endorsement, recommendation, or favoring by the United States Government or any agency thereof. The views and opinions of authors expressed herein do not necessarily state or reflect those of the United States Government or any agency thereof. 
Environmental Sciences Division Center for Radiation Protection Knowledge

\begin{abstract}
Air Exchange Rate Impact on Actinon, Thoron, and Radon Activity Equilibrium Factor and Inhalation Fractional Equilibrium Factor Determination in Vapor Intrusion Risk and Dose Models

Ethan A. Asano, Fredrick G. Dolislager, Karessa L. Manning, Debra J. Stewart, Katie A. Noto, Hayden J. Ringer, Caleigh E. Samuels, Leslie D. Galloway, Anthony Q. Armstrong, Michael B. Bellamy
\end{abstract}

Date Published: September 2020

Prepared by

OAK RIDGE NATIONAL LABORATORY

Oak Ridge, TN 37831-6283

managed by

UT-BATTELLE, LLC

for the

US DEPARTMENT OF ENERGY

under contract DE-AC05-00OR22725 



\section{ERRATA}

Summary of changes:

\begin{tabular}{|c|l|l||}
\hline Page & Section & Description of Change \\
\hline \hline 3 & Chapter 2, Last paragraph & $\begin{array}{l}\text { Text about the Feq not being used in calculations was updated to } \\
\text { reflect that Feq is used in conversions of activity to working levels. }\end{array}$ \\
\hline A-3 & Appendix A, Table A-2 & $\begin{array}{l}\text { The missing air exchange rate }(\mathrm{ACH}) \text { of "8" was added, and } \\
\text { duplicate air exchange rates of } 800 \text { and 900 were removed. }\end{array}$ \\
\hline B-3 & Appendix B, Table B-2 & $\begin{array}{l}\text { The missing air exchange rate }(\mathrm{ACH}) \text { of "8" was added, and } \\
\text { duplicate air exchange rates of } 800 \text { and 900 were removed. }\end{array}$ \\
\hline
\end{tabular}





\section{CONTENTS}

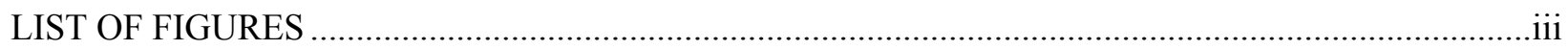

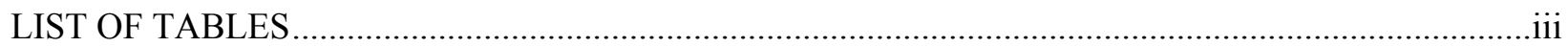

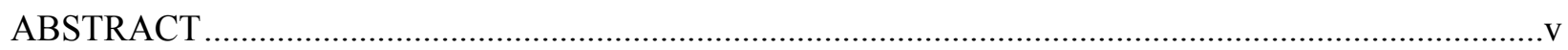

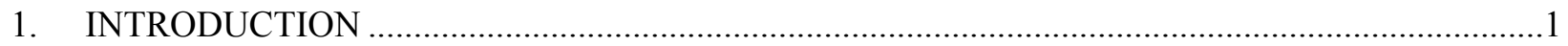

2. CALCULATION OF ACTINON, THORON, AND RADON INHALATION FRACTIONAL

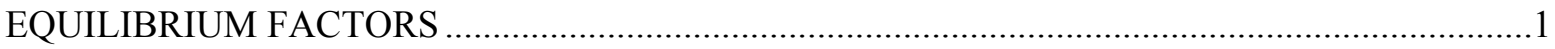

3. CALCULATION OF RADON, THORON, AND ACTINON ACTIVITY EQUILIBRIUM

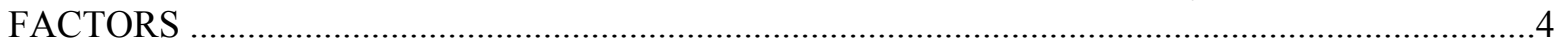

4. USE OF RADON, THORON, AND ACTINON ACTIVITY EQUILIBRIUM FACTORS IN

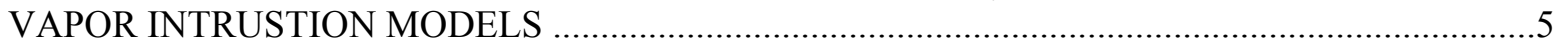

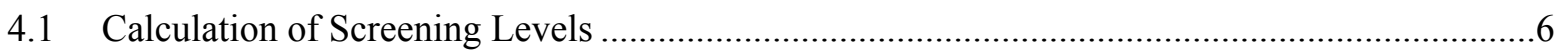

4.2 Calculation of Excess Lifetime Cancer Risk and Annual Dose..............................................6

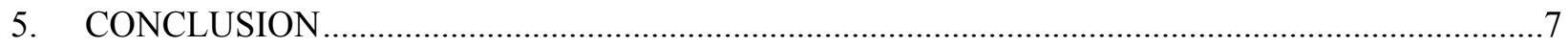

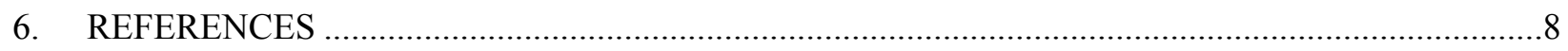

APPENDIX A. ACTIVITY EQUILIBRIUM FACTORS BASED ON AIR EXCHANGE RATE .........A-1

APPENDIX B. INHALATION FRACTIONAL EQUILIBRIUM FACTORS BASED ON AIR

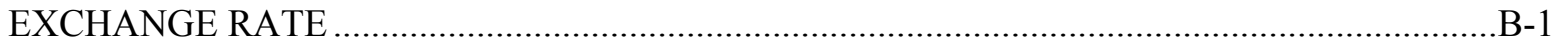

APPENDIX C. ACTINON, THORON, AND RADON DECAY SERIES DATA …..............................

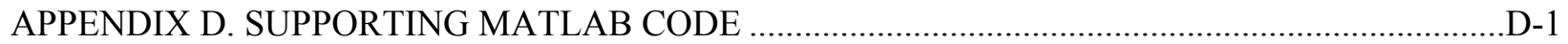

APPENDIX E. ACTIVITY EQUILIBRIUM FACTOR CALCULATION SUPPLEMENTAL

GUIDE

E-1

\section{LIST OF FIGURES}

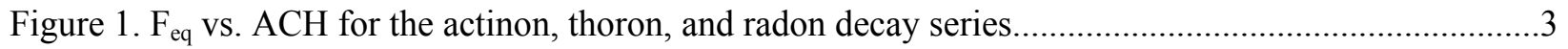

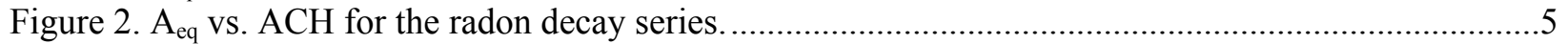

\section{LIST OF TABLES}

Table A-1. Actinon Activity Equilibrium Factors Based on Air Changes Per Hour................................A-1

Table A-2: Thoron Activity Equilibrium Factors Based on Air Changes Per Hour..................................3

Table A-3: Radon Activity Equilibrium Factors Based on Air Changes Per Hour ....................................5

Table B-1: Actinon Inhalation Fractional Equilibrium Factors Based on Air Changes Per Hour.............B-1

Table B-2: Thoron Inhalation Fractional Equilibrium Factors Based on Air Changes Per Hour................3

Table B-3: Radon Inhalation Fractional Equilibrium Factors Based on Air Changes Per Hour .................

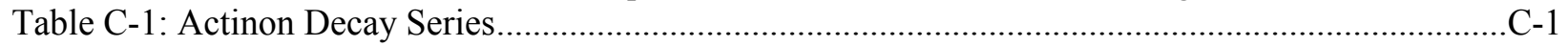

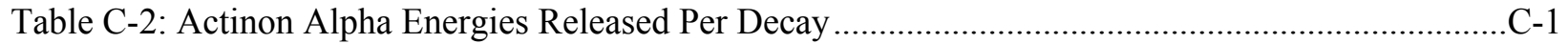

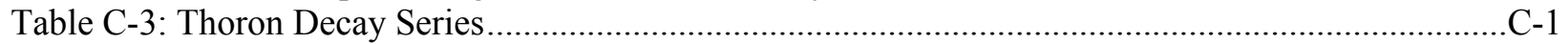

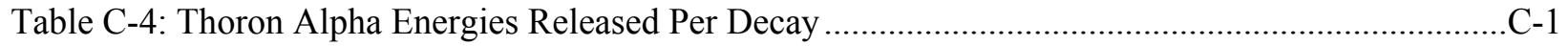

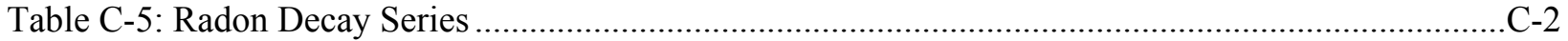

Table C-6: Radon Alpha Energies Released Per Decay .......................................................................... 



\begin{abstract}
Exposure to the radioactive isotopes of radon are of high concern and, when present in vapor form, may pose a significant risk to humans in an indoor air environment. Understanding the radiometric and physical properties of radon radioisotopes are important for risk assessment. Little is known of the chemical properties of radon because of the short half-lives of all of its isotopes (NCRP, 1988). Indoor air screening levels (SL) based on an annual target risk or dose limit were determined for the actinon ( $\mathrm{Rn}-219)$, thoron ( $\mathrm{Rn}-220)$, and radon (Rn-222) decay series. The SLs are based on inhalation and submersion in gas cloud exposure routes for residential and commercial settings. The SLs are analogous to preliminary remediation goals (PRGs) and dose compliance concentrations (DCCs), where the isotope-specific values are in units of activity concentration (activity per unit volume). If the concentration of a parent isotope of radon or its progeny is found to exceed the SL, then further action to ensure cleanup of the contaminant may be necessary. In residential and commercial settings, the SLs will vary based on the air exchange rate present. In this study, a computational method in MATLAB was developed to determine the impact of the air exchange rate on the activity equilibrium factor $\left(\mathrm{A}_{\mathrm{eq}}\right)$ and the inhalation fractional equilibrium factor $\left(\mathrm{F}_{\mathrm{eq}}\right)$. Both factors are values that reflect the equilibrium concentrations of progeny to parent in the air. These factors have a direct impact on PRGs, DCCs, excess lifetime cancer risk, and annual dose; they are analyzed in subsequent sections along with applications in vapor intrusion risk and dose models.
\end{abstract}





\section{INTRODUCTION}

Radon is a naturally occurring inert gas produced from the decay of uranium found in rocks and soil. Outdoor exposure to radon is usually of little concern for public health, since it is present in trace amounts in the Earth's atmosphere. When radon escapes natural media such as groundwater, rocks, and soil, however, it can easily concentrate in indoor environments in vapor form. Thus, radon vapor intrusion screening levels (RVISLs) were developed to assess the contamination level from the actinon, thoron, and radon decay series in indoor air residential and commercial environments.

Known radiometric properties, such as half-life and decay energy emission data for the actinon, thoron, and radon decay series, were used to determine the activity equilibrium factors $\left(\mathrm{A}_{\mathrm{eq}}\right)$ and inhalation fractional equilibrium factors $\left(\mathrm{F}_{\text {eq }}\right)$ for vapor intrusion risk and dose models. Decay series data, along with corresponding alpha energy emissions for actinon, thoron, and radon, are provided in Appendix C (ICRP, 2008). The concentration ratios between radon and its progeny indoors (i.e., the indoor $\mathrm{F}_{\mathrm{eq}}$ ) is very much influenced by ventilation, exhalation, solid-particle concentration, and surface deposition of radon progeny (Chen \& Harley, 2018). Exhalation, solidparticle concentration, and surface deposition were neglected in this study, for simplification. Air quality was also neglected, and it was assumed that actinon, thoron, radon, and subsequent progeny were uniformly distributed over the air volume.

The methods for determining $\mathrm{A}_{\mathrm{eq}}$ and $\mathrm{F}_{\mathrm{eq}}$ in this study are based upon theoretical calculations rather than experimental. Known experimental methods for calculating the individual activity concentrations of thoron and radon progeny in air, such as the Tsivoglou and Cliff methods (NCRP, 1988), are inapplicable for comparison in this study, since they include the surface deposition of individual progeny by using filtered air samples to obtain activity concentrations.

\section{CALCULATION OF ACTINON, THORON, AND RADON INHALATION FRACTIONAL EQUILIBRIUM FACTORS}

The indoor air concentration is dependent upon the air exchange rate within a given volume. The air exchange rate, otherwise known as air changes per hour $(\mathrm{ACH})$, is defined as the ratio of airflow and the total volume of air given by the following equation:

$$
A C H\left(h r^{-1}\right)=\frac{\text { Volume of Air Exchanged per Hour }(L / h r)}{\text { Total Volume of Air }(L)}[1]
$$

The ACH can be a building-specific measurement, to provide a more accurate air contamination exposure assessment. The ACH is the exchange rate of indoor air with outdoor air, an important determinant for entry of outdoor air contaminants, and for removal of indoor-emitted air contaminants. There is no EPA guidance on recommended methods for determining ACH. Breen (2014) presents an overview and critical analysis of the scientific literature on empirical and physical air exchange rate models for residential and commercial buildings, which are feasible for exposure assessments. Models are included for the three types of airflows that can occur across building envelopes: leakage, natural ventilation, and mechanical ventilation. The paper 
provides guidance for selecting the preferable air exchange rate model based on available data, desired temporal resolution, types of airflows, and types of buildings included in the exposure assessment. The US EPA Indoor Air Quality website (EPA, 2019a) also provides information on HVAC use in buildings. There are also more detailed papers on particular air exchange measurement approaches (i.e., by placing tubes that emit and collect perfluorocarbon tracers throughout a building). The measurement of air exchange rates can be done by placing tubes that emit and collect perfluorocarbon tracers throughout a building. Guidance on placement of the tubes can be found in Thornburg (2005). As an example, an air exchange rate of 0.18 would mean that eighteen percent of a given volume of air is exchanged per hour. The ACH and Feq are both used for estimating the level of detriment from radon inhalation. Feq is a unitless measurement of the disequilibrium associated with the decay of radon and its subsequent progeny. In addition, Feq is useful for calculating the internal dose, because most of the dose received is not from the parent itself but rather the alpha energies emitted from its progeny. Therefore, the dose per unit radon gas activity is greatly dependent on the state of equilibrium of the decay products (CDC, 2018). The equation for Feq is shown below:

$$
\left(F_{e q}\right)_{i}=\frac{\text { Total Progeny Alpha Energy Emitted }(\mathrm{MeV})}{\text { Total Progeny Alpha Energy Emitted at Equilibrium (MeV) }}, \text { [2] }
$$

where $i$ represents an arbitrary air exchange rate. The total progeny alpha energy emitted in the numerator of equation [2] may be calculated at any air exchange rate. The total progeny alpha energy emitted in the denominator of equation [2] is at a state of equilibrium, where no air exchanges are taking place. When there is no air exchange, the numerator and denominator of equation [2] are equal, and $\mathrm{F}_{\mathrm{eq}}$ is a value of one. As the air exchange rate in a given volume increases, $\mathrm{F}_{\text {eq }}$ decreases and vice-versa. Figure 1 below displays the trends in $\mathrm{F}_{\text {eq }} \mathrm{Vs}$. ACH for the actinon, thoron, and radon decay series. 


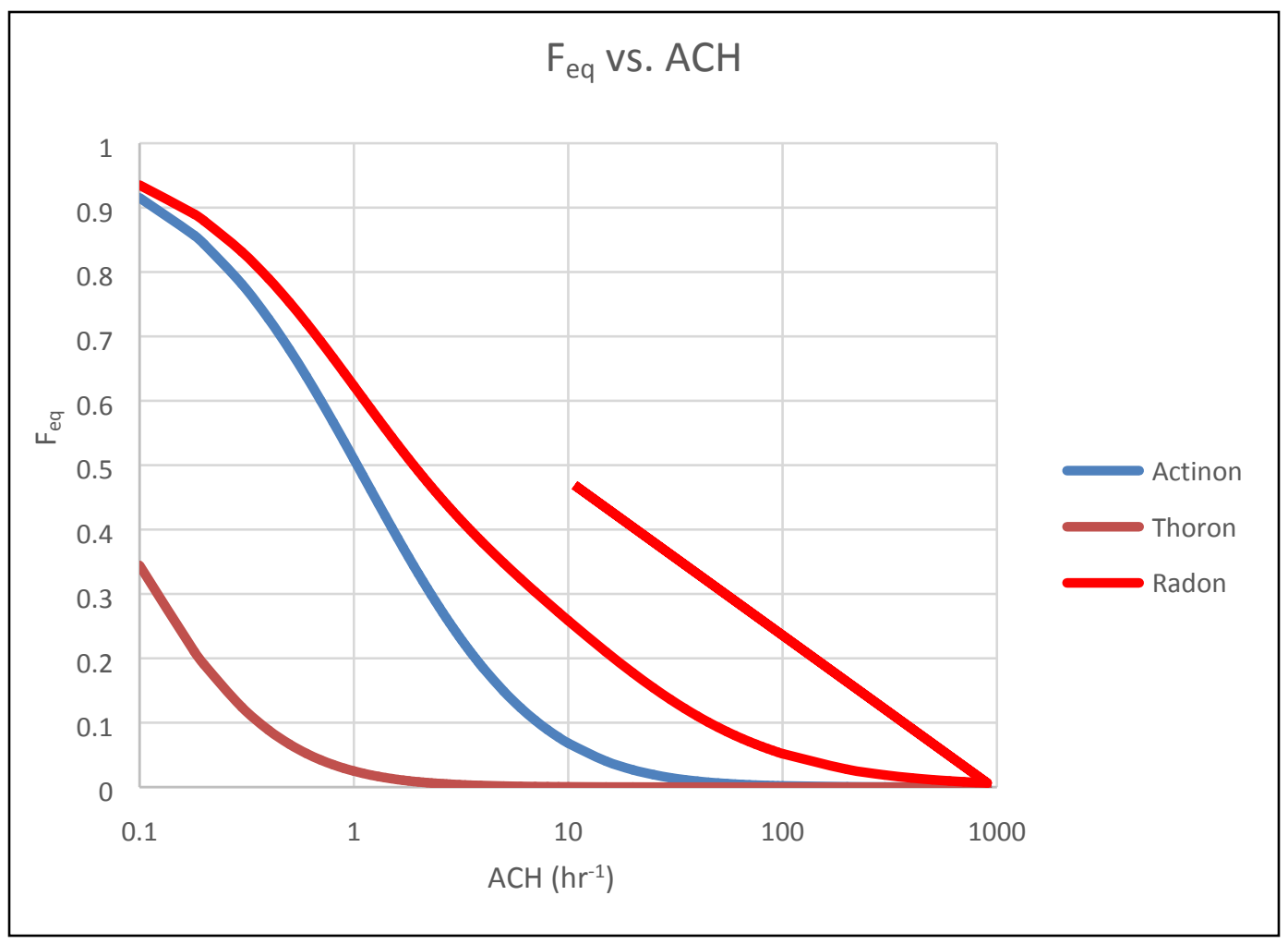

Figure 1. $\mathrm{F}_{\mathrm{eq}} \mathrm{vs.} \mathrm{ACH}$ for the actinon, thoron, and radon decay series. (As the air exchange rate increases, the inhalation fractional equilibrium factor $\left(F_{e q}\right)$ decreases. This decrease results in less carcinogenic risk.)

$F_{e q}$ is related to the potential alpha energy concentration (PAEC), which is the kinetic energy potentially released in a unit volume of air from alpha particles emitted by the short-lived radioactive progeny of Rn-222 or Rn-220 (DOE, 2008). In this study, the PAEC definition is applied to the actinon progeny in order to calculate its $\mathrm{F}_{\text {eq }}$. The method used to calculate $\mathrm{F}_{\mathrm{eq}}$ is the ratio of the PAEC in the existing mixture to that which would exist if all short-lived daughters were in equilibrium with the radon present (NCRP, 1988), which is reflected in equation [2] above.

The alpha energies released per decay for each isotope of the actinon, thoron, and radon decay series were multiplied by their respective $\mathrm{A}_{\mathrm{eq}}$ to obtain the PAECs used in equation [2]. The alpha energies, obtained from ICRP 107, are shown in Appendix C. All alpha energies were included in the analysis, regardless of fractional contribution. The only exception is for radon, where $\mathrm{Pb}-210$ and subsequent progeny are not included. The assumption is that the relatively long half-life of $\mathrm{Pb}-210$ will allow it to settle out of the air.

$\mathrm{F}_{\mathrm{eq}}$ is only used in the conversions of activity to working levels and is presented in the calculator output (EPA, 2019b) for informational purposes. Historically, $\mathrm{F}_{\mathrm{eq}}$ was used to describe the disequilibrium of the progeny in air and was significant in evaluating the risk and dose. $\mathrm{F}_{\mathrm{eq}}$ has been researched for radon and thoron in various dwellings for many years (Chen \& Harley, 2018). The analysis in this report presents $\mathrm{F}_{\text {eq }}$ for actinon, which is not currently available in literature. 


\section{CALCULATION OF RADON, THORON, AND ACTINON ACTIVITY EQUILIBRIUM FACTORS}

The $\mathrm{A}_{\mathrm{eq}}$ is defined as the concentration ratio of progeny and parent activity at a given air exchange rate by the following equation:

$$
\left(A_{e q}\right)_{i}=\frac{\text { Activity Concentration of Progeny }(p C i / L)}{\text { Activity Concentration of Parent }(p C i / L)}
$$

where $i$ represents an arbitrary air exchange rate. The numerator of equation [3] includes the activity concentrations of progeny within a specific radon decay chain. The denominator of equation [3] includes the activity concentration of the parent and is always equal to one picocurie per liter of air. At zero air exchanges, $A_{\text {eq }}$ values for all radon progeny are equal to one; they decrease as the air exchange rate increases.

$\mathrm{A}_{\mathrm{eq}}$ is utilized in the RVISL air equations to calculate risk and dose for inhalation and submersion in gas cloud exposure routes. The inhalation exposure route is based on internal dose resulting from breathing contaminated air. The submersion in gas cloud exposure routes is based on exposure to radiation emitted from contaminants in the air. The initial risks and doses (analogous to PRGs) assume that the radon decay chain members are in equilibrium with each other without any air exchanges present. Therefore, $\mathrm{A}_{\mathrm{eq}}$ modifies the initial risks and doses for inhalation and submersion by accounting for the disequilibrium between radon and its progeny due to decay and air exchanges that are present. $A_{\text {eq }}$ factors for both inhalation and submersion are equivalent at any given air exchange rate and involve only the decay of each radionuclide in air. $\mathrm{A}_{\mathrm{eq}}$ does not account for the radiation type (i.e., alpha, beta) since that is already accounted for in the derivation of slope factors and dose conversion factors in the RVISL tool (Bellamy et al., 2014; Leggett \& Bellamy, 2017). Appendix A provides $A_{\text {eq }}$ values for actinon, thoron, and radon for several air exchange rates. Default values of 0.18 and 0.6 air exchanges per hour were selected for the resident and industrial worker land uses, respectively, from Table 19-25 (resident) and Table 19-30 (commercial) of the "Update for Chapter 19 of the Exposure Factors Handbook" (EPA, 2018) using the tenth percent values. Figure 2 displays the trends in $\mathrm{A}_{\mathrm{eq}} \mathrm{vs}$. $\mathrm{ACH}$ at $0, .18$, and 0.6 air changes per hour for the radon decay series. 


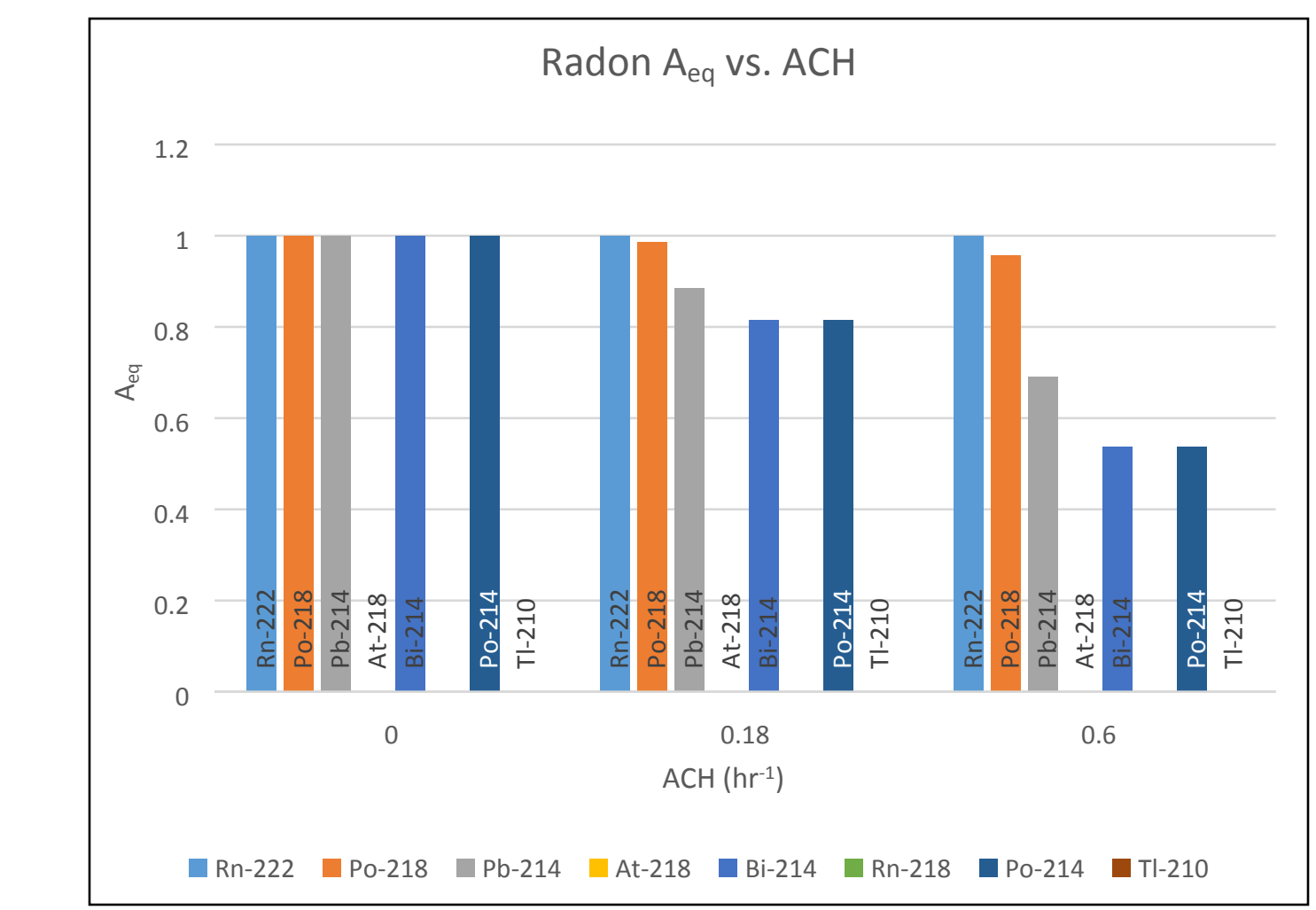

\section{Figure 2. $A_{\text {eq }}$ vs. ACH for the radon decay series. $\left(\mathrm{A}_{\mathrm{eq}}\right.$ at $0,0.18$ (default residential land use), and 0.6 (default industrial worker) $\mathrm{ACH}$ are shown above with progeny decreasing as the $\mathrm{ACH}$ increases.)}

$\mathrm{A}_{\mathrm{eq}}$ values for the actinon, thoron, and radon decay series were calculated using MATLAB, as shown in Appendix D, for air exchange rates ranging from zero to nine hundred per hour over a period of two hundred hours at each air exchange rate. An ordinary differential equation (ODE) solver was ultimately utilized to calculate $\mathrm{A}_{\mathrm{eq}}$ for each radioisotope of the actinon, thoron, and radon decay series. A supplemental guide is provided in Appendix E, which includes a detailed explanation of the methodology used to calculate $A_{\text {eq }}$ values in MATLAB.

\section{USE OF RADON, THORON, AND ACTINON ACTIVITY EQUILIBRIUM FACTORS IN VAPOR INTRUSTION MODELS}

The $\mathrm{A}_{\mathrm{eq}}$ values generated from this study are used in risk and dose assessment models for intrusion of radon into buildings. They can be applied to evaluate risk or dose due to inhalation and external exposure to ionizing radiation in RVISL equations. For each radon radioisotope, the calculator identifies all the progeny in the chain. For thoron and actinon, the full chain is used; for radon, the chain is stopped at $\mathrm{Pb}-210$ due to its long half-life. The individual RVISLs for each progeny are combined with the parent on a fractional basis, as influenced by the air exchange rate and the resulting $\mathrm{A}_{\mathrm{eq}}$. The fractional basis is determined by branching fractions where a progeny may decay into more than one isotope, and the $\mathrm{A}_{\mathrm{eq}}$ is determined based on the air exchange rate. The resulting RVISL is now based on the air exchange rate influenced equilibrium of the full chain. It is assumed that the parent is continually being introduced into the structure. The equations found in 4.1 and 4.2 depict the use of $\mathrm{A}_{\mathrm{eq}}$ in residential air RVISL equations. Inhalation risk and dose coefficients 
have not been calculated for actinon because the half-life is too short to derive such coefficients. Therefore, risk and dose due to inhalation are only evaluated for thoron and radon.

\subsection{CALCULATION OF SCREENING LEVELS}

$A_{e q}$ is used in the inverse sum of reciprocals, as shown below in equations [5] and [6], to account for the air exchange rate and the decay of actinon, thoron, and radon. The RVISLs for inhalation and submersion may be based on either a target risk or an annual dose limit for a receptor. The

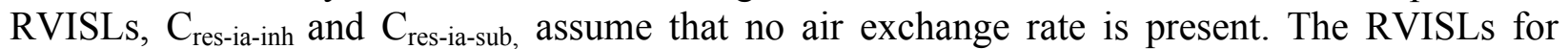
inhalation and submersion neglect the risk and dose contribution from progeny before intake. After intake of the parent radionuclide, however, the risk and dose from progeny in the body is included (Leggett \& Bellamy, 2017). The total risk and dose-based RVISL for inhalation and submersion, $\mathrm{C}_{\text {res-ia-tot, }}$ is calculated using equation [4], with an inverse sum of reciprocals that utilizes the outputs from equations [5] and [6] for actinon, thoron, and radon.

\section{Resident Air Risk Equations}

$$
\begin{aligned}
& C_{r e s-i a-\operatorname{tot}}\left(p C i / m^{3}\right)=\frac{1}{\frac{1}{C_{i n h}\left(p C i / m^{3}\right)}+\frac{1}{C_{s u b}\left(p C i / m^{3}\right)}}[4] \\
& C_{i n h}\left(p C i / m^{3}\right)=\frac{1}{\left(\sum_{i=1}^{n} \frac{1}{\left(\frac{\left.C_{r e s-i a-i n h\left(p C i / m^{3}\right.}\right)}{A_{e q}}\right)}\right)} \\
& C_{s u b}\left(p C i / m^{3}\right)=\underbrace{\left.\sum_{i=1}^{n} \frac{1}{\left(\frac{{ }^{C} r e s-i a-s u b\left(p C i / m^{3}\right)}{A_{e q}}\right)}\right)^{[6]}}_{i}
\end{aligned}
$$

\subsection{CALCULATION OF EXCESS LIFETIME CANCER RISK AND ANNUAL DOSE}

The inhalation and submersion excess lifetime cancer risk (ELCR) and dose apply $\mathrm{A}_{\text {eq }}$ to the chronic daily intakes (CDI), as shown below in equations [7] and [8], to account for the air exchange rate and the decay of actinon, thoron, and radon. The CDIs for inhalation and submersion vary based on duration of exposure. The ELCR and dose for inhalation and submersion neglect the risk and dose contribution from progeny before intake. After intake of the parent radionuclide, however, the risk and dose from progeny in the body is included (Leggett \& Bellamy, 2017). The 
total ELCR and dose, ELCR tot and Dose tot $_{\text {, }}$ are calculated using the sum of inhalation and submersion ELCR or dose for actinon, thoron, and radon.

\section{Excess Lifetime Cancer Risk}

$$
E L C R_{\text {tot }}=E L C R_{\text {inh }}+E L C R_{\text {sub }},[7]
$$

where:

$$
E L C R_{\text {inh }}=\sum_{i=1}^{n}\left(C D I(p C i) \times S F_{i}(r i s k / p C i) \times A_{e q}\right)_{i}
$$

and:

$$
E L C R_{\text {sub }}=\sum_{i=1}^{n}\left(\operatorname{CDI}\left(p C i-\text { year } / m^{3}\right) \times S F_{\text {sub }}\left(\frac{\text { risk } / \text { year }}{p C i / m^{3}}\right) \times A_{e q}\right)_{i}
$$

where $\mathrm{i}=$ the number of progeny in the decay series.

$$
\begin{gathered}
\text { Annual Dose } \\
\text { Dose }_{\text {tot }}\left(\frac{\text { mrem }}{\text { year }}\right)=\text { Dose }_{\text {inh }}\left(\frac{\text { mrem }}{\text { year }}\right)+\text { Dose }_{\text {sub }}\left(\frac{\text { mrem }}{\text { year }}\right),
\end{gathered}
$$

where:

$$
\text { Dose }_{\text {inh }}\left(\frac{\text { mrem }}{\text { year }}\right)=\sum_{i=1}^{n}\left(C D I\left(\frac{p C i}{\text { year }}\right) \times D C F_{i}\left(\frac{\text { mrem }}{\text { year }}\right) \times A_{e q}\right)_{i}
$$

and:

$$
\text { Dose }_{\text {sub }}\left(\frac{\text { mrem }}{\text { year }}\right)=\sum_{i=1}^{n}\left(C D I\left(\frac{p C i}{m^{3}}\right) \times D C F_{\text {sub }}\left(\frac{\text { mrem } / \text { year }}{p C i / m^{3}}\right) \times A_{e q}\right)_{i}
$$

where $\mathrm{i}=$ the number of progeny in the decay series.

\section{CONCLUSION}

Screening levels for the actinon, thoron, and radon decay series were calculated for an annual target risk and dose limit to a receptor. In an indoor air setting, the screening levels depend on the values of the air exchange rate and the resulting $A_{\text {eq. }}$. A computational method in MATLAB was developed to determine the impact of the air exchange rate on $\mathrm{A}_{\mathrm{eq}}$ and $\mathrm{F}_{\mathrm{eq}}$ for indoor air residential and commercial settings. As the air exchange rate increases, $A_{\text {eq }}$, inhalation $F_{\text {eq }}$, and risk to receptors decrease. The $A_{e q}$ is applicable in vapor intrusion risk and dose models for inhalation and submersion, while the $\mathrm{F}_{\mathrm{eq}}$ is only applicable for calculating the dose from inhalation. 


\section{REFERENCES}

Bellamy, M.; Finklea, L.; Dolislager, F.; Eckerman, K. 2014. Calculation of Slope Factors and Dose Coefficients. ORNL/TM-2013/00. Oak Ridge National Laboratory, Oak Ridge, TN. https://epa-prgs.ornl.gov/radionuclides/SlopesandDosesFinal.pdf.

Breen, MS et al. 2014. A review of air exchange rate models for air pollution exposure assessments. J Expo Sci Environ Epidemiol, 24(6):555-63. https://epa-

visl.ornl.gov/radionuclides/download/Breen.pdf.

CDC. 2018. Dose Conversion Factors for Radon WLM, Revision 05. Centers for Disease Control and Prevention. https://www.cdc.gov/niosh/ocas/pdfs/tibs/dc-t11-r5-508.pdf.

Chen, J; Harley, N. 2018. A Review of Indoor and Outdoor Radon Equilibrium Factors-Part 1: Rn-222. Health Physics Journal, 115(4):490-499.

DOE. 2008. Internal Dosimetry. DOE-STD-1121. U.S. Department of Energy, Washington, D.C.

EPA. 2018. Update for Chapter 19 of the Exposure Factors Handbook. Environmental Protection Agency, Washington, D.C.

EPA. 2019a. Heating, Ventilation and Air-conditioning Text Modules for the Indoor Air Quality Building Education and Assessment Model. Indoor Air Quality. https://www.epa.gov/indoor-airquality-iaq/heating-ventilation-and-air-conditioning-text-modules-indoor-air-quality.

EPA. 2019b. Radon Vapor Intrusion Screening Level Calculator. https://epavisl.ornl.gov/radionuclides/.

ICRP. 2008. Nuclear Decay Data for Dosimetric Calculations. ICRP Publication 107. Ann. ICRP, $38(3)$.

Legget, Richard W. and Bellamy, Michael B. 2017. Radon Cancer Risk Coefficients \& AgeSpecific Effective Dose Coefficients. ORNL-/TM-2017/47. Oak Ridge National Laboratory, Oak ridge, TN.

NCRP. 1988. Measurement of Radon Daughters in Air. Report No. 97. National Council on Radiation Protection and Measurements, Bethesda, MD.

Thornburg, Jonathan W. 2005. Research Operating Procedure 10 for Placement of PFT Emitter and Collection Tubes in DEARS. Rev. \#04. RTI International, Research Triangle Park, NC. https://epa-visl.ornl.gov/radionuclides/download/ROP10.pdf. 
APPENDIX A. ACTIVITY EQUILIBRIUM FACTORS BASED ON AIR EXCHANGE RATE 

Table A-1. Actinon Activity Equilibrium Factors Based on Air Changes Per Hour

\begin{tabular}{|c|c|c|c|c|c|c|}
\hline \multirow[b]{2}{*}{$\begin{array}{c}\mathrm{ACH}^{\mathrm{a}} \\
\left(\mathrm{hr}^{-1}\right)\end{array}$} & \multicolumn{6}{|c|}{ Activity Equilibrium Factor } \\
\hline & Rn-219 & Po-215 & Pb-211 & Bi-211 & Tl-207 & Po-211 \\
\hline 0 & $1.000 E+00$ & $9.972 \mathrm{E}-01$ & $1.000 \mathrm{E}+00$ & $1.000 E+00$ & $9.970 \mathrm{E}-01$ & $2.800 \mathrm{E}-03$ \\
\hline 0.1 & $1.000 \mathrm{E}+00$ & $9.968 \mathrm{E}-01$ & $9.201 \mathrm{E}-01$ & $9.154 \mathrm{E}-01$ & $9.023 \mathrm{E}-01$ & $2.500 \mathrm{E}-03$ \\
\hline 0.18 & $1.000 \mathrm{E}+00$ & $1.000 \mathrm{E}+00$ & $8.648 \mathrm{E}-01$ & 8.569E-01 & 8.371E-01 & 2.400E-03 \\
\hline 0.2 & $1.000 E+00$ & $9.964 \mathrm{E}-01$ & $8.521 \mathrm{E}-01$ & 8.434E-01 & 8.220E-01 & 2.300E-03 \\
\hline 0.3 & $1.000 E+00$ & $9.960 \mathrm{E}-01$ & 7.934E-01 & 7.813E-01 & 7.530E-01 & 2.200E-03 \\
\hline 0.4 & $1.000 \mathrm{E}+00$ & $9.956 \mathrm{E}-01$ & $7.422 \mathrm{E}-01$ & 7.273E-01 & 6.933E-01 & 2.000E-03 \\
\hline 0.5 & $1.000 E+00$ & $9.951 E-01$ & $6.973 \mathrm{E}-01$ & $6.798 \mathrm{E}-01$ & $6.410 \mathrm{E}-01$ & $1.900 \mathrm{E}-03$ \\
\hline 0.6 & $1.000 \mathrm{E}+00$ & $9.946 \mathrm{E}-01$ & $6.575 \mathrm{E}-01$ & $6.378 \mathrm{E}-01$ & $5.949 \mathrm{E}-01$ & $1.800 \mathrm{E}-03$ \\
\hline 0.7 & $1.000 \mathrm{E}+00$ & $9.904 \mathrm{E}-01$ & $6.220 \mathrm{E}-01$ & $6.004 \mathrm{E}-01$ & $5.541 \mathrm{E}-01$ & $1.700 \mathrm{E}-03$ \\
\hline 0.8 & $1.000 \mathrm{E}+00$ & $9.917 \mathrm{E}-01$ & 5.901E-01 & $5.668 \mathrm{E}-01$ & $5.176 \mathrm{E}-01$ & $1.600 \mathrm{E}-03$ \\
\hline 0.9 & $1.000 E+00$ & 9.929E-01 & $5.613 \mathrm{E}-01$ & 5.365E-01 & $4.848 \mathrm{E}-01$ & $1.500 \mathrm{E}-03$ \\
\hline 1 & $1.000 \mathrm{E}+00$ & $9.940 \mathrm{E}-01$ & $5.352 \mathrm{E}-01$ & $5.091 \mathrm{E}-01$ & 4.553E-01 & $1.400 \mathrm{E}-03$ \\
\hline 1.1 & $1.000 \mathrm{E}+00$ & $9.950 \mathrm{E}-01$ & 5.115E-01 & $4.841 \mathrm{E}-01$ & 4.286E-01 & $1.300 \mathrm{E}-03$ \\
\hline 1.2 & $1.000 \mathrm{E}+00$ & $9.959 \mathrm{E}-01$ & 4.897E-01 & $4.612 \mathrm{E}-01$ & $4.042 \mathrm{E}-01$ & $1.300 \mathrm{E}-03$ \\
\hline 1.3 & $1.000 \mathrm{E}+00$ & $9.967 \mathrm{E}-01$ & 4.697E-01 & 4.403E-01 & $3.820 \mathrm{E}-01$ & $1.200 \mathrm{E}-03$ \\
\hline 1.4 & $1.000 \mathrm{E}+00$ & $9.974 \mathrm{E}-01$ & 4.513E-01 & $4.210 \mathrm{E}-01$ & 3.617E-01 & $1.200 \mathrm{E}-03$ \\
\hline 1.5 & $1.000 E+00$ & $9.981 \mathrm{E}-01$ & 4.343E-01 & 4.032E-01 & $3.430 \mathrm{E}-01$ & 1.100E-03 \\
\hline 1.6 & $1.000 \mathrm{E}+00$ & 9.987E-01 & 4.185E-01 & 3.867E-01 & 3.257E-01 & $1.100 \mathrm{E}-03$ \\
\hline 1.7 & $1.000 \mathrm{E}+00$ & 9.992E-01 & $4.038 \mathrm{E}-01$ & 3.714E-01 & $3.098 \mathrm{E}-01$ & $1.000 \mathrm{E}-03$ \\
\hline 1.8 & $1.000 \mathrm{E}+00$ & 9.997E-01 & $3.902 \mathrm{E}-01$ & $3.571 \mathrm{E}-01$ & $2.951 \mathrm{E}-01$ & $1.000 \mathrm{E}-03$ \\
\hline 1.9 & $1.000 \mathrm{E}+00$ & $1.000 E+00$ & $3.774 \mathrm{E}-01$ & $3.438 \mathrm{E}-01$ & $2.814 \mathrm{E}-01$ & $9.000 \mathrm{E}-04$ \\
\hline 2 & $1.000 \mathrm{E}+00$ & $1.000 E+00$ & $3.654 \mathrm{E}-01$ & 3.313E-01 & $2.687 \mathrm{E}-01$ & $9.000 \mathrm{E}-04$ \\
\hline 2.1 & $1.000 \mathrm{E}+00$ & $1.000 E+00$ & $3.541 \mathrm{E}-01$ & 3.196E-01 & $2.568 \mathrm{E}-01$ & $9.000 \mathrm{E}-04$ \\
\hline 2.2 & $1.000 E+00$ & $1.000 \mathrm{E}+00$ & $3.436 \mathrm{E}-01$ & 3.086E-01 & $2.457 \mathrm{E}-01$ & $9.000 \mathrm{E}-04$ \\
\hline 2.3 & $1.000 \mathrm{E}+00$ & $1.000 \mathrm{E}+00$ & 3.336E-01 & $2.983 \mathrm{E}-01$ & 2.353E-01 & 8.000E-04 \\
\hline 2.4 & $1.000 \mathrm{E}+00$ & $1.000 E+00$ & $3.242 \mathrm{E}-01$ & $2.886 \mathrm{E}-01$ & $2.256 \mathrm{E}-01$ & 8.000E-04 \\
\hline 2.5 & $1.000 \mathrm{E}+00$ & 1.000E+00 & 3.153E-01 & 2.794E-01 & 2.165E-01 & 8.000E-04 \\
\hline 2.6 & $1.000 \mathrm{E}+00$ & $1.000 E+00$ & 3.069E-01 & 2.707E-01 & 2.079E-01 & 7.000E-04 \\
\hline 2.7 & $1.000 E+00$ & $1.000 E+00$ & 2.990E-01 & 2.625E-01 & $1.998 \mathrm{E}-01$ & 7.000E-04 \\
\hline 2.8 & $1.000 \mathrm{E}+00$ & 1.000E+00 & $2.914 \mathrm{E}-01$ & 2.547E-01 & $1.922 \mathrm{E}-01$ & 7.000E-04 \\
\hline 2.9 & $1.000 E+00$ & $1.000 \mathrm{E}+00$ & $2.842 \mathrm{E}-01$ & $2.473 \mathrm{E}-01$ & $1.850 \mathrm{E}-01$ & 7.000E-04 \\
\hline 3 & $1.000 E+00$ & $1.000 E+00$ & 2.773E-01 & $2.403 \mathrm{E}-01$ & $1.782 \mathrm{E}-01$ & 7.000E-04 \\
\hline 3.1 & $1.000 \mathrm{E}+00$ & $1.000 \mathrm{E}+00$ & $2.708 \mathrm{E}-01$ & $2.336 \mathrm{E}-01$ & $1.718 \mathrm{E}-01$ & 6.000E-04 \\
\hline 3.2 & $1.000 \mathrm{E}+00$ & $1.000 \mathrm{E}+00$ & $2.646 \mathrm{E}-01$ & $2.272 \mathrm{E}-01$ & $1.657 \mathrm{E}-01$ & 6.000E-04 \\
\hline 3.3 & $1.000 E+00$ & $1.000 E+00$ & $2.586 \mathrm{E}-01$ & $2.211 \mathrm{E}-01$ & $1.599 \mathrm{E}-01$ & 6.000E-04 \\
\hline 3.4 & $1.000 \mathrm{E}+00$ & $1.000 \mathrm{E}+00$ & $2.530 \mathrm{E}-01$ & $2.153 \mathrm{E}-01$ & $1.544 \mathrm{E}-01$ & 6.000E-04 \\
\hline 3.5 & $1.000 \mathrm{E}+00$ & $1.000 \mathrm{E}+00$ & $2.475 \mathrm{E}-01$ & 2.097E-01 & $1.492 \mathrm{E}-01$ & $6.000 \mathrm{E}-04$ \\
\hline 3.6 & $1.000 E+00$ & $1.000 E+00$ & $2.423 \mathrm{E}-01$ & $2.044 \mathrm{E}-01$ & $1.443 \mathrm{E}-01$ & 6.000E-04 \\
\hline
\end{tabular}




\begin{tabular}{|c|c|c|c|c|c|c|}
\hline & & & Activity Equil & ibrium Factor & & \\
\hline $\begin{array}{l}\text { ACF" } \\
\left(\mathbf{h} \mathbf{r}^{-1}\right)\end{array}$ & Rn-219 & Po-215 & $\mathrm{Pb}-211$ & Bi-211 & Tl-207 & Po-211 \\
\hline 3.7 & $1.000 \mathrm{E}+00$ & $1.000 E+00$ & 2.373E-01 & 1.994E-01 & 1.395E-01 & 5.000E-04 \\
\hline 3.8 & $1.000 \mathrm{E}+00$ & $1.000 E+00$ & 2.325E-01 & $1.945 \mathrm{E}-01$ & $1.350 \mathrm{E}-01$ & $5.000 \mathrm{E}-04$ \\
\hline 3.9 & $1.000 E+00$ & $1.000 E+00$ & 2.279E-01 & $1.898 \mathrm{E}-01$ & $1.308 \mathrm{E}-01$ & $5.000 \mathrm{E}-04$ \\
\hline 4 & $1.000 E+00$ & $1.000 E+00$ & 2.235E-01 & $1.853 \mathrm{E}-01$ & 1.267E-01 & $5.000 \mathrm{E}-04$ \\
\hline 5 & $1.000 \mathrm{E}+00$ & 9.967E-01 & 1.871E-01 & $1.488 \mathrm{E}-01$ & $9.430 \mathrm{E}-02$ & $4.000 \mathrm{E}-04$ \\
\hline 6 & $1.000 \mathrm{E}+00$ & $9.928 \mathrm{E}-01$ & 1.609E-01 & 1.230E-01 & $7.260 \mathrm{E}-02$ & $3.000 \mathrm{E}-04$ \\
\hline 7 & $1.000 E+00$ & 9.997E-01 & 1.412E-01 & $1.038 \mathrm{E}-01$ & $5.740 \mathrm{E}-02$ & $3.000 \mathrm{E}-04$ \\
\hline 8 & $1.000 E+00$ & $1.000 E+00$ & 1.257E-01 & $8.910 \mathrm{E}-02$ & $4.630 \mathrm{E}-02$ & 2.000E-04 \\
\hline 9 & $1.000 \mathrm{E}+00$ & $1.000 E+00$ & 1.133E-01 & 7.750E-02 & $3.800 \mathrm{E}-02$ & 2.000E-04 \\
\hline 10 & $1.000 E+00$ & $9.961 \mathrm{E}-01$ & $1.032 \mathrm{E}-01$ & $6.810 \mathrm{E}-02$ & $3.160 \mathrm{E}-02$ & 2.000E-04 \\
\hline 15 & $1.000 \mathrm{E}+00$ & $9.954 \mathrm{E}-01$ & 7.120E-02 & 4.020E-02 & $1.470 \mathrm{E}-02$ & 1.000E-04 \\
\hline 20 & $1.000 \mathrm{E}+00$ & $9.947 \mathrm{E}-01$ & $5.430 \mathrm{E}-02$ & $2.680 \mathrm{E}-02$ & $8.100 \mathrm{E}-03$ & $1.000 \mathrm{E}-04$ \\
\hline 25 & $1.000 E+00$ & 9.939E-01 & 4.390E-02 & 1.920E-02 & 4.900E-03 & $1.000 \mathrm{E}-04$ \\
\hline 30 & $1.000 E+00$ & $9.932 \mathrm{E}-01$ & $3.680 \mathrm{E}-02$ & $1.450 \mathrm{E}-02$ & $3.200 \mathrm{E}-03$ & $0.000 E+00$ \\
\hline 35 & $1.000 E+00$ & $9.923 \mathrm{E}-01$ & $3.170 \mathrm{E}-02$ & 1.130E-02 & $2.300 \mathrm{E}-03$ & $0.000 E+00$ \\
\hline 40 & $1.000 E+00$ & $9.915 \mathrm{E}-01$ & $2.780 \mathrm{E}-02$ & $9.100 \mathrm{E}-03$ & $1.600 \mathrm{E}-03$ & $0.000 E+00$ \\
\hline 45 & $1.000 \mathrm{E}+00$ & $9.906 \mathrm{E}-01$ & $2.480 \mathrm{E}-02$ & 7.500E-03 & $1.200 \mathrm{E}-03$ & $0.000 E+00$ \\
\hline 50 & $1.000 E+00$ & 9.897E-01 & $2.240 \mathrm{E}-02$ & $6.300 \mathrm{E}-03$ & $9.000 \mathrm{E}-04$ & $0.000 \mathrm{E}+00$ \\
\hline 55 & $1.000 E+00$ & $9.888 \mathrm{E}-01$ & $2.040 \mathrm{E}-02$ & $5.300 \mathrm{E}-03$ & 7.000E-04 & $0.000 E+00$ \\
\hline 60 & $1.000 \mathrm{E}+00$ & $9.878 \mathrm{E}-01$ & 1.870E-02 & 4.600E-03 & $6.000 \mathrm{E}-04$ & $0.000 E+00$ \\
\hline 65 & $1.000 E+00$ & $9.868 \mathrm{E}-01$ & $1.730 \mathrm{E}-02$ & 4.000E-03 & $5.000 \mathrm{E}-04$ & $0.000 E+00$ \\
\hline 70 & $1.000 \mathrm{E}+00$ & $9.891 \mathrm{E}-01$ & $1.600 \mathrm{E}-02$ & 3.500E-03 & $4.000 \mathrm{E}-04$ & $0.000 \mathrm{E}+00$ \\
\hline 75 & $1.000 E+00$ & $9.882 \mathrm{E}-01$ & $1.500 \mathrm{E}-02$ & $3.100 \mathrm{E}-03$ & $3.000 \mathrm{E}-04$ & $0.000 \mathrm{E}+00$ \\
\hline 80 & $1.000 E+00$ & $9.873 E-01$ & $1.400 \mathrm{E}-02$ & 2.700E-03 & $3.000 \mathrm{E}-04$ & $0.000 E+00$ \\
\hline 85 & $1.000 E+00$ & $9.864 \mathrm{E}-01$ & $1.320 \mathrm{E}-02$ & $2.500 \mathrm{E}-03$ & $2.000 \mathrm{E}-04$ & $0.000 E+00$ \\
\hline 90 & $1.000 E+00$ & $9.854 \mathrm{E}-01$ & $1.250 \mathrm{E}-02$ & $2.200 \mathrm{E}-03$ & $2.000 \mathrm{E}-04$ & $0.000 \mathrm{E}+00$ \\
\hline 95 & $1.000 \mathrm{E}+00$ & $9.844 \mathrm{E}-01$ & $1.180 \mathrm{E}-02$ & 2.000E-03 & $2.000 \mathrm{E}-04$ & $0.000 \mathrm{E}+00$ \\
\hline 100 & $1.000 \mathrm{E}+00$ & $9.833 \mathrm{E}-01$ & $1.120 \mathrm{E}-02$ & $1.800 \mathrm{E}-03$ & $1.000 \mathrm{E}-04$ & $0.000 \mathrm{E}+00$ \\
\hline 200 & $1.000 E+00$ & $9.730 \mathrm{E}-01$ & $5.600 \mathrm{E}-03$ & $5.000 \mathrm{E}-04$ & $0.000 E+00$ & $0.000 E+00$ \\
\hline 300 & $1.000 E+00$ & $9.629 E-01$ & $3.700 \mathrm{E}-03$ & $2.000 \mathrm{E}-04$ & $0.000 E+00$ & $0.000 E+00$ \\
\hline 400 & $1.000 \mathrm{E}+00$ & $9.464 \mathrm{E}-01$ & 2.700E-03 & 1.000E-04 & $0.000 E+00$ & $0.000 \mathrm{E}+00$ \\
\hline 500 & $1.000 E+00$ & $9.277 \mathrm{E}-01$ & $2.100 \mathrm{E}-03$ & 1.000E-04 & $0.000 E+00$ & $0.000 E+00$ \\
\hline 600 & $1.000 E+00$ & $9.224 \mathrm{E}-01$ & $1.800 \mathrm{E}-03$ & $1.000 \mathrm{E}-04$ & $0.000 E+00$ & $0.000 E+00$ \\
\hline 700 & $1.000 \mathrm{E}+00$ & $9.078 \mathrm{E}-01$ & $1.500 \mathrm{E}-03$ & $0.000 E+00$ & $0.000 \mathrm{E}+00$ & $0.000 E+00$ \\
\hline 800 & $1.000 E+00$ & $8.951 \mathrm{E}-01$ & 1.300E-03 & $0.000 E+00$ & $0.000 E+00$ & $0.000 E+00$ \\
\hline 900 & $1.000 \mathrm{E}+00$ & 8.831E-01 & $1.100 \mathrm{E}-03$ & $0.000 E+00$ & $0.000 E+00$ & $0.000 \mathrm{E}+00$ \\
\hline
\end{tabular}

a 0.18 is the residential default $\mathrm{ACH}$, and 0.6 is the commercial/industrial default $\mathrm{ACH}$. 
Table A-2: Thoron Activity Equilibrium Factors Based on Air Changes Per Hour

\begin{tabular}{|c|c|c|c|c|c|c|}
\hline \multirow[b]{2}{*}{$\begin{array}{c}\mathbf{A C H}^{\mathbf{a}} \\
\left(\mathbf{h r}^{-1}\right)\end{array}$} & \multicolumn{6}{|c|}{ Activity Equilibrium Factor } \\
\hline & Rn-220 & Po-216 & $\mathrm{Pb}-212$ & Bi-212 & Po-212 & Tl-208 \\
\hline 0 & $1.000 E+00$ & $1.000 \mathrm{E}+00$ & $1.000 \mathrm{E}+00$ & $1.000 E+00$ & 6.410E-01 & 3.590E-01 \\
\hline 0.1 & $1.000 E+00$ & $1.000 E+00$ & 3.945E-01 & 3.443E-01 & 2.207E-01 & 1.227E-01 \\
\hline 0.18 & $1.000 E+00$ & $1.000 \mathrm{E}+00$ & 2.657E-01 & 2.106E-01 & $1.350 \mathrm{E}-01$ & 7.460E-02 \\
\hline 0.2 & $1.000 \mathrm{E}+00$ & $1.000 E+00$ & 2.457E-01 & 1.903E-01 & $1.220 \mathrm{E}-01$ & 6.730E-02 \\
\hline 0.3 & $1.000 \mathrm{E}+00$ & $1.000 E+00$ & $1.784 \mathrm{E}-01$ & $1.242 \mathrm{E}-01$ & 7.960E-02 & 4.360E-02 \\
\hline 0.4 & $1.000 E+00$ & $1.000 E+00$ & $1.401 \mathrm{E}-01$ & 8.850E-02 & 5.670E-02 & 3.090E-02 \\
\hline 0.5 & $1.000 \mathrm{E}+00$ & $1.000 E+00$ & $1.153 \mathrm{E}-01$ & 6.670E-02 & $4.280 \mathrm{E}-02$ & 2.310E-02 \\
\hline 0.6 & $1.000 \mathrm{E}+00$ & $1.000 \mathrm{E}+00$ & 9.790E-02 & 5.230E-02 & $3.350 \mathrm{E}-02$ & 1.800E-02 \\
\hline 0.7 & $1.000 \mathrm{E}+00$ & $1.000 \mathrm{E}+00$ & 8.510E-02 & $4.220 \mathrm{E}-02$ & $2.700 \mathrm{E}-02$ & $1.440 \mathrm{E}-02$ \\
\hline 0.8 & $1.000 E+00$ & $1.000 \mathrm{E}+00$ & $7.530 \mathrm{E}-02$ & $3.480 \mathrm{E}-02$ & $2.230 \mathrm{E}-02$ & $1.180 \mathrm{E}-02$ \\
\hline 0.9 & $1.000 \mathrm{E}+00$ & 9.999E-01 & 6.750E-02 & 2.920E-02 & $1.870 \mathrm{E}-02$ & $9.800 \mathrm{E}-03$ \\
\hline 1 & $1.000 E+00$ & $9.999 \mathrm{E}-01$ & $6.120 \mathrm{E}-02$ & 2.490E-02 & $1.600 \mathrm{E}-02$ & $8.300 \mathrm{E}-03$ \\
\hline 1.1 & $1.000 E+00$ & $9.999 \mathrm{E}-01$ & $5.590 \mathrm{E}-02$ & $2.150 \mathrm{E}-02$ & $1.380 \mathrm{E}-02$ & $7.100 \mathrm{E}-03$ \\
\hline 1.2 & $1.000 E+00$ & $9.999 \mathrm{E}-01$ & $5.150 \mathrm{E}-02$ & $1.870 \mathrm{E}-02$ & $1.200 \mathrm{E}-02$ & $6.200 \mathrm{E}-03$ \\
\hline 1.3 & $1.000 E+00$ & 9.999E-01 & 4.770E-02 & $1.650 \mathrm{E}-02$ & $1.060 \mathrm{E}-02$ & $5.400 \mathrm{E}-03$ \\
\hline 1.4 & $1.000 \mathrm{E}+00$ & $9.999 \mathrm{E}-01$ & $4.450 \mathrm{E}-02$ & 1.460E-02 & $9.400 \mathrm{E}-03$ & $4.800 \mathrm{E}-03$ \\
\hline 1.5 & $1.000 E+00$ & 9.999E-01 & 4.160E-02 & 1.310E-02 & $8.400 \mathrm{E}-03$ & 4.200E-03 \\
\hline 1.6 & $1.000 E+00$ & $9.999 \mathrm{E}-01$ & $3.910 \mathrm{E}-02$ & 1.170E-02 & 7.500E-03 & $3.800 \mathrm{E}-03$ \\
\hline 1.7 & $1.000 E+00$ & 9.999E-01 & $3.690 \mathrm{E}-02$ & $1.060 \mathrm{E}-02$ & $6.800 \mathrm{E}-03$ & $3.400 \mathrm{E}-03$ \\
\hline 1.8 & $1.000 E+00$ & $9.999 \mathrm{E}-01$ & $3.490 \mathrm{E}-02$ & $9.600 \mathrm{E}-03$ & $6.200 \mathrm{E}-03$ & $3.100 \mathrm{E}-03$ \\
\hline 1.9 & $1.000 E+00$ & 9.999E-01 & $3.310 \mathrm{E}-02$ & $8.800 \mathrm{E}-03$ & $5.600 \mathrm{E}-03$ & $2.800 \mathrm{E}-03$ \\
\hline 2 & $1.000 E+00$ & 9.999E-01 & $3.150 \mathrm{E}-02$ & 8.100E-03 & $5.200 \mathrm{E}-03$ & $2.500 \mathrm{E}-03$ \\
\hline 2.1 & $1.000 E+00$ & $9.999 \mathrm{E}-01$ & 3.010E-02 & 7.400E-03 & $4.800 \mathrm{E}-03$ & 2.300E-03 \\
\hline 2.2 & $1.000 E+00$ & 9.999E-01 & $2.880 \mathrm{E}-02$ & $6.800 \mathrm{E}-03$ & 4.400E-03 & $2.100 \mathrm{E}-03$ \\
\hline 2.3 & $1.000 E+00$ & $9.999 \mathrm{E}-01$ & $2.750 \mathrm{E}-02$ & $6.300 \mathrm{E}-03$ & 4.100E-03 & $1.900 \mathrm{E}-03$ \\
\hline 2.4 & $1.000 E+00$ & 9.999E-01 & $2.640 \mathrm{E}-02$ & 5.900E-03 & $3.800 \mathrm{E}-03$ & $1.800 \mathrm{E}-03$ \\
\hline 2.5 & $1.000 E+00$ & 9.999E-01 & $2.540 \mathrm{E}-02$ & 5.500E-03 & $3.500 \mathrm{E}-03$ & $1.700 \mathrm{E}-03$ \\
\hline 2.6 & $1.000 E+00$ & $9.998 \mathrm{E}-01$ & $2.440 \mathrm{E}-02$ & 5.100E-03 & 3.300E-03 & $1.500 \mathrm{E}-03$ \\
\hline 2.7 & $1.000 E+00$ & $9.998 \mathrm{E}-01$ & $2.360 \mathrm{E}-02$ & 4.800E-03 & 3.100E-03 & $1.400 \mathrm{E}-03$ \\
\hline 2.8 & $1.000 E+00$ & $9.998 \mathrm{E}-01$ & $2.270 \mathrm{E}-02$ & 4.500E-03 & $2.900 \mathrm{E}-03$ & $1.300 \mathrm{E}-03$ \\
\hline 2.9 & $1.000 E+00$ & $9.998 \mathrm{E}-01$ & $2.200 \mathrm{E}-02$ & 4.200E-03 & $2.700 \mathrm{E}-03$ & $1.200 \mathrm{E}-03$ \\
\hline 3 & $1.000 E+00$ & $9.998 \mathrm{E}-01$ & $2.120 \mathrm{E}-02$ & 4.000E-03 & $2.500 \mathrm{E}-03$ & $1.200 \mathrm{E}-03$ \\
\hline 3.1 & $1.000 E+00$ & $9.998 \mathrm{E}-01$ & $2.060 \mathrm{E}-02$ & 3.700E-03 & $2.400 \mathrm{E}-03$ & $1.100 \mathrm{E}-03$ \\
\hline 3.2 & $1.000 E+00$ & $9.998 \mathrm{E}-01$ & $1.990 \mathrm{E}-02$ & 3.500E-03 & $2.300 \mathrm{E}-03$ & $1.000 \mathrm{E}-03$ \\
\hline 3.3 & $1.000 E+00$ & $9.998 \mathrm{E}-01$ & $1.940 \mathrm{E}-02$ & 3.300E-03 & $2.100 \mathrm{E}-03$ & $1.000 \mathrm{E}-03$ \\
\hline 3.4 & $1.000 E+00$ & $9.998 \mathrm{E}-01$ & $1.880 \mathrm{E}-02$ & 3.200E-03 & $2.000 \mathrm{E}-03$ & $9.000 \mathrm{E}-04$ \\
\hline 3.5 & $1.000 \mathrm{E}+00$ & $9.998 \mathrm{E}-01$ & $1.830 \mathrm{E}-02$ & 3.000E-03 & $1.900 \mathrm{E}-03$ & $9.000 \mathrm{E}-04$ \\
\hline 3.6 & $1.000 E+00$ & $9.998 \mathrm{E}-01$ & $1.780 \mathrm{E}-02$ & $2.800 \mathrm{E}-03$ & $1.800 \mathrm{E}-03$ & 8.000E-04 \\
\hline
\end{tabular}




\begin{tabular}{|c|c|c|c|c|c|c|}
\hline & \multicolumn{6}{|c|}{ Activity Equilibrium Factor } \\
\hline $\begin{array}{c}\mathrm{ACH}_{\left(\mathbf{h} \mathbf{r}^{-1}\right)} \\
\text { a }\end{array}$ & Rn-220 & Po-216 & $\mathrm{Pb}-212$ & Bi-212 & Po-212 & Tl-208 \\
\hline 3.7 & $1.000 \mathrm{E}+00$ & $9.998 \mathrm{E}-01$ & $1.730 \mathrm{E}-02$ & 2.700E-03 & $1.700 \mathrm{E}-03$ & 8.000E-04 \\
\hline 3.8 & $1.000 E+00$ & $9.998 \mathrm{E}-01$ & $1.690 \mathrm{E}-02$ & $2.600 \mathrm{E}-03$ & $1.700 \mathrm{E}-03$ & 7.000E-04 \\
\hline 3.9 & $1.000 \mathrm{E}+00$ & $9.998 \mathrm{E}-01$ & $1.640 \mathrm{E}-02$ & $2.500 \mathrm{E}-03$ & $1.600 \mathrm{E}-03$ & 7.000E-04 \\
\hline 4 & $1.000 E+00$ & $9.998 \mathrm{E}-01$ & $1.600 \mathrm{E}-02$ & $2.300 \mathrm{E}-03$ & $1.500 \mathrm{E}-03$ & 7.000E-04 \\
\hline 5 & $1.000 \mathrm{E}+00$ & 9.997E-01 & $1.290 \mathrm{E}-02$ & $1.600 \mathrm{E}-03$ & $1.000 \mathrm{E}-03$ & $4.000 \mathrm{E}-04$ \\
\hline 6 & $1.000 \mathrm{E}+00$ & 9.997E-01 & $1.070 \mathrm{E}-02$ & $1.100 \mathrm{E}-03$ & $7.000 \mathrm{E}-04$ & 3.000E-04 \\
\hline 7 & $1.000 E+00$ & $9.996 \mathrm{E}-01$ & $9.200 \mathrm{E}-03$ & 8.000E-04 & $5.000 \mathrm{E}-04$ & 2.000E-04 \\
\hline 8 & $1.000 E+00$ & $9.995 \mathrm{E}-01$ & 8.100E-03 & $6.000 \mathrm{E}-04$ & $4.000 \mathrm{E}-04$ & $1.000 \mathrm{E}-04$ \\
\hline 9 & $1.000 E+00$ & $9.995 \mathrm{E}-01$ & 7.200E-03 & $5.000 \mathrm{E}-04$ & $3.000 \mathrm{E}-04$ & $1.000 \mathrm{E}-04$ \\
\hline 10 & $1.000 E+00$ & $9.994 \mathrm{E}-01$ & $6.500 \mathrm{E}-03$ & 4.000E-04 & $3.000 \mathrm{E}-04$ & $1.000 \mathrm{E}-04$ \\
\hline 15 & $1.000 E+00$ & 9.991E-01 & 4.300E-03 & 2.000E-04 & $1.000 \mathrm{E}-04$ & $0.000 E+00$ \\
\hline 20 & $1.000 E+00$ & $9.988 \mathrm{E}-01$ & $3.200 \mathrm{E}-03$ & $1.000 \mathrm{E}-04$ & $1.000 \mathrm{E}-04$ & $0.000 E+00$ \\
\hline 25 & $1.000 \mathrm{E}+00$ & $9.985 \mathrm{E}-01$ & $2.600 \mathrm{E}-03$ & $1.000 \mathrm{E}-04$ & $0.000 E+00$ & $0.000 E+00$ \\
\hline 30 & $1.000 \mathrm{E}+00$ & $9.983 \mathrm{E}-01$ & $2.200 \mathrm{E}-03$ & $0.000 E+00$ & $0.000 E+00$ & $0.000 E+00$ \\
\hline 35 & $1.000 E+00$ & $9.980 \mathrm{E}-01$ & $1.900 \mathrm{E}-03$ & $0.000 E+00$ & $0.000 E+00$ & $0.000 E+00$ \\
\hline 40 & $1.000 E+00$ & $9.977 \mathrm{E}-01$ & $1.600 \mathrm{E}-03$ & $0.000 E+00$ & $0.000 E+00$ & $0.000 E+00$ \\
\hline 45 & $1.000 E+00$ & $9.974 \mathrm{E}-01$ & $1.400 \mathrm{E}-03$ & $0.000 E+00$ & $0.000 E+00$ & $0.000 E+00$ \\
\hline 50 & $1.000 E+00$ & $9.971 \mathrm{E}-01$ & $1.300 \mathrm{E}-03$ & $0.000 \mathrm{E}+00$ & $0.000 E+00$ & $0.000 E+00$ \\
\hline 55 & $1.000 E+00$ & $9.968 \mathrm{E}-01$ & $1.200 \mathrm{E}-03$ & $0.000 \mathrm{E}+00$ & $0.000 E+00$ & $0.000 E+00$ \\
\hline 60 & $1.000 E+00$ & $9.965 \mathrm{E}-01$ & 1.100E-03 & $0.000 E+00$ & $0.000 E+00$ & $0.000 E+00$ \\
\hline 65 & $1.000 E+00$ & $9.962 \mathrm{E}-01$ & 1.000E-03 & $0.000 E+00$ & $0.000 E+00$ & $0.000 E+00$ \\
\hline 70 & $1.000 \mathrm{E}+00$ & $9.959 \mathrm{E}-01$ & $9.000 \mathrm{E}-04$ & $0.000 E+00$ & $0.000 E+00$ & $0.000 E+00$ \\
\hline 75 & $1.000 E+00$ & $9.957 \mathrm{E}-01$ & $9.000 \mathrm{E}-04$ & $0.000 E+00$ & $0.000 E+00$ & $0.000 E+00$ \\
\hline 80 & $1.000 E+00$ & $9.954 \mathrm{E}-01$ & $8.000 \mathrm{E}-04$ & $0.000 E+00$ & $0.000 E+00$ & $0.000 E+00$ \\
\hline 85 & $1.000 \mathrm{E}+00$ & $9.951 \mathrm{E}-01$ & 8.000E-04 & $0.000 E+00$ & $0.000 E+00$ & $0.000 E+00$ \\
\hline 90 & $1.000 E+00$ & $9.948 \mathrm{E}-01$ & 7.000E-04 & $0.000 E+00$ & $0.000 E+00$ & $0.000 E+00$ \\
\hline 95 & $1.000 E+00$ & $9.945 \mathrm{E}-01$ & 7.000E-04 & $0.000 E+00$ & $0.000 E+00$ & $0.000 E+00$ \\
\hline 100 & $1.000 E+00$ & $9.942 \mathrm{E}-01$ & $6.000 \mathrm{E}-04$ & $0.000 E+00$ & $0.000 E+00$ & $0.000 E+00$ \\
\hline 200 & $1.000 \mathrm{E}+00$ & $9.885 \mathrm{E}-01$ & $3.000 \mathrm{E}-04$ & $0.000 E+00$ & $0.000 E+00$ & $0.000 E+00$ \\
\hline 300 & $1.000 E+00$ & $9.829 \mathrm{E}-01$ & $2.000 \mathrm{E}-04$ & $0.000 E+00$ & $0.000 E+00$ & $0.000 E+00$ \\
\hline 400 & $1.000 E+00$ & $9.773 \mathrm{E}-01$ & $2.000 \mathrm{E}-04$ & $0.000 E+00$ & $0.000 E+00$ & $0.000 E+00$ \\
\hline 500 & $1.000 \mathrm{E}+00$ & $9.718 \mathrm{E}-01$ & 1.000E-04 & $0.000 E+00$ & $0.000 E+00$ & $0.000 E+00$ \\
\hline 600 & $1.000 E+00$ & $9.663 \mathrm{E}-01$ & $1.000 \mathrm{E}-04$ & $0.000 E+00$ & $0.000 E+00$ & $0.000 E+00$ \\
\hline 700 & $1.000 E+00$ & $9.609 E-01$ & $1.000 \mathrm{E}-04$ & $0.000 E+00$ & $0.000 E+00$ & $0.000 E+00$ \\
\hline 800 & $1.000 \mathrm{E}+00$ & $9.556 \mathrm{E}-01$ & $1.000 \mathrm{E}-04$ & $0.000 E+00$ & $0.000 E+00$ & $0.000 E+00$ \\
\hline 900 & $1.000 \mathrm{E}+00$ & $9.503 \mathrm{E}-01$ & 1.000E-04 & $0.000 E+00$ & $0.000 E+00$ & $0.000 E+00$ \\
\hline
\end{tabular}

a 0.18 is the residential default $\mathrm{ACH}$, and 0.6 is the commercial/industrial default $\mathrm{ACH}$. 
Table A-3: Radon Activity Equilibrium Factors Based on Air Changes Per Hour

\begin{tabular}{|c|c|c|c|c|c|c|c|c|}
\hline & \multicolumn{8}{|c|}{ Activity Equilibrium Factor } \\
\hline $\begin{array}{c}\mathbf{A C H}^{\mathbf{a}} \\
\left(\mathbf{h r ^ { - 1 } )}\right.\end{array}$ & Rn-222 & Po-218 & $\mathrm{Pb}-214$ & At-218 & Bi-214 & Rn-218 & Po-214 & Tl-210 \\
\hline 0 & $1.000 E+00$ & $1.000 E+00$ & $1.000 E+00$ & $2.000 \mathrm{E}-04$ & $1.000 E+00$ & 2.000E-07 & $1.000 E+00$ & $2.100 \mathrm{E}-04$ \\
\hline 0.1 & $1.000 \mathrm{E}+00$ & $9.926 \mathrm{E}-01$ & $9.325 \mathrm{E}-01$ & $1.985 \mathrm{E}-04$ & 8.901E-01 & $1.985 \mathrm{E}-07$ & 8.901E-01 & $1.863 \mathrm{E}-04$ \\
\hline 0.18 & $1.000 \mathrm{E}+00$ & $9.868 \mathrm{E}-01$ & $8.842 E-01$ & 1.973E-04 & 8.143E-01 & 1.973E-07 & 8.143E-01 & $1.700 \mathrm{E}-04$ \\
\hline 0.2 & $1.000 \mathrm{E}+00$ & $9.853 \mathrm{E}-01$ & $8.728 \mathrm{E}-01$ & $1.970 \mathrm{E}-04$ & 7.968E-01 & 1.970E-07 & 7.968E-01 & $1.663 \mathrm{E}-04$ \\
\hline 0.3 & $1.000 \mathrm{E}+00$ & $9.781 \mathrm{E}-01$ & 8.197E-01 & $1.956 \mathrm{E}-04$ & 7.169E-01 & $1.956 \mathrm{E}-07$ & 7.169E-01 & 1.492E-04 \\
\hline 0.4 & $1.000 \mathrm{E}+00$ & 9.710E-01 & 7.720E-01 & $1.942 \mathrm{E}-04$ & $6.482 \mathrm{E}-01$ & $1.942 \mathrm{E}-07$ & $6.482 \mathrm{E}-01$ & 1.344E-04 \\
\hline 0.5 & $1.000 \mathrm{E}+00$ & $9.641 \mathrm{E}-01$ & 7.291E-01 & $1.928 \mathrm{E}-04$ & $5.885 \mathrm{E}-01$ & $1.928 \mathrm{E}-07$ & $5.885 \mathrm{E}-01$ & 1.217E-04 \\
\hline 0.6 & $1.000 \mathrm{E}+00$ & $9.572 \mathrm{E}-01$ & $6.903 \mathrm{E}-01$ & $1.914 \mathrm{E}-04$ & 5.365E-01 & $1.914 \mathrm{E}-07$ & $5.365 \mathrm{E}-01$ & $1.106 \mathrm{E}-04$ \\
\hline 0.7 & $1.000 \mathrm{E}+00$ & $9.504 \mathrm{E}-01$ & $6.550 \mathrm{E}-01$ & $1.900 \mathrm{E}-04$ & 4.908E-01 & $1.900 \mathrm{E}-07$ & 4.908E-01 & 1.009E-04 \\
\hline 0.8 & $1.000 \mathrm{E}+00$ & $9.437 \mathrm{E}-01$ & $6.227 \mathrm{E}-01$ & $1.887 \mathrm{E}-04$ & 4.505E-01 & $1.887 \mathrm{E}-07$ & 4.505E-01 & $9.229 \mathrm{E}-05$ \\
\hline 0.9 & $1.000 \mathrm{E}+00$ & $9.371 \mathrm{E}-01$ & 5.931E-01 & $1.873 \mathrm{E}-04$ & 4.147E-01 & $1.873 \mathrm{E}-07$ & 4.147E-01 & $8.471 \mathrm{E}-05$ \\
\hline 1 & $1.000 \mathrm{E}+00$ & $9.306 \mathrm{E}-01$ & $5.659 \mathrm{E}-01$ & $1.860 \mathrm{E}-04$ & 3.829E-01 & $1.860 \mathrm{E}-07$ & 3.829E-01 & 7.797E-05 \\
\hline 1.1 & $1.000 \mathrm{E}+00$ & $9.242 \mathrm{E}-01$ & $5.408 \mathrm{E}-01$ & $1.847 \mathrm{E}-04$ & 3.545E-01 & 1.847E-07 & $3.545 \mathrm{E}-01$ & 7.196E-05 \\
\hline 1.2 & $1.000 \mathrm{E}+00$ & $9.179 E-01$ & $5.176 \mathrm{E}-01$ & $1.834 \mathrm{E}-04$ & 3.289E-01 & $1.834 \mathrm{E}-07$ & $3.289 \mathrm{E}-01$ & $6.658 \mathrm{E}-05$ \\
\hline 1.3 & $1.000 \mathrm{E}+00$ & $9.117 \mathrm{E}-01$ & 4.961E-01 & $1.822 \mathrm{E}-04$ & 3.059E-01 & $1.822 \mathrm{E}-07$ & 3.059E-01 & $6.174 \mathrm{E}-05$ \\
\hline 1.4 & $1.000 \mathrm{E}+00$ & $9.055 \mathrm{E}-01$ & 4.760E-01 & $1.809 \mathrm{E}-04$ & $2.852 \mathrm{E}-01$ & $1.809 \mathrm{E}-07$ & $2.852 \mathrm{E}-01$ & $5.738 \mathrm{E}-05$ \\
\hline 1.5 & $1.000 \mathrm{E}+00$ & 8.994E-01 & $4.574 \mathrm{E}-01$ & 1.797E-04 & 2.664E-01 & 1.797E-07 & 2.664E-01 & $5.343 E-05$ \\
\hline 1.6 & $1.000 \mathrm{E}+00$ & 8.934E-01 & 4.399E-01 & $1.785 \mathrm{E}-04$ & 2.492E-01 & $1.785 \mathrm{E}-07$ & 2.492E-01 & 4.985E-05 \\
\hline 1.7 & $1.000 \mathrm{E}+00$ & $8.875 E-01$ & 4.235E-01 & $1.773 \mathrm{E}-04$ & 2.337E-01 & 1.773E-07 & 2.337E-01 & 4.659E-05 \\
\hline 1.8 & $1.000 E+00$ & 8.817E-01 & $4.082 \mathrm{E}-01$ & $1.761 \mathrm{E}-04$ & 2.194E-01 & $1.761 \mathrm{E}-07$ & 2.194E-01 & 4.362E-05 \\
\hline 1.9 & $1.000 \mathrm{E}+00$ & 8.759E-01 & $3.938 \mathrm{E}-01$ & $1.750 \mathrm{E}-04$ & $2.064 \mathrm{E}-01$ & $1.750 \mathrm{E}-07$ & $2.064 \mathrm{E}-01$ & 4.091E-05 \\
\hline 2 & $1.000 E+00$ & 8.703E-01 & $3.802 \mathrm{E}-01$ & $1.738 \mathrm{E}-04$ & $1.944 \mathrm{E}-01$ & $1.738 \mathrm{E}-07$ & $1.944 \mathrm{E}-01$ & $3.842 \mathrm{E}-05$ \\
\hline 2.1 & $1.000 \mathrm{E}+00$ & 8.647E-01 & $3.674 \mathrm{E}-01$ & $1.727 \mathrm{E}-04$ & $1.834 \mathrm{E}-01$ & 1.727E-07 & $1.834 \mathrm{E}-01$ & $3.613 \mathrm{E}-05$ \\
\hline 2.2 & $1.000 \mathrm{E}+00$ & 8.591E-01 & $3.553 \mathrm{E}-01$ & $1.716 \mathrm{E}-04$ & $1.732 \mathrm{E}-01$ & $1.716 \mathrm{E}-07$ & $1.732 \mathrm{E}-01$ & $3.403 E-05$ \\
\hline 2.3 & $1.000 \mathrm{E}+00$ & 8.537E-01 & $3.439 \mathrm{E}-01$ & $1.705 \mathrm{E}-04$ & $1.638 \mathrm{E}-01$ & $1.705 \mathrm{E}-07$ & $1.638 \mathrm{E}-01$ & $3.209 E-05$ \\
\hline 2.4 & $1.000 \mathrm{E}+00$ & 8.483E-01 & 3.331E-01 & $1.694 \mathrm{E}-04$ & 1.551E-01 & $1.694 \mathrm{E}-07$ & $1.551 \mathrm{E}-01$ & 3.030E-05 \\
\hline 2.5 & $1.000 \mathrm{E}+00$ & 8.429E-01 & $3.228 \mathrm{E}-01$ & $1.683 \mathrm{E}-04$ & $1.471 \mathrm{E}-01$ & $1.683 \mathrm{E}-07$ & $1.471 \mathrm{E}-01$ & $2.865 \mathrm{E}-05$ \\
\hline 2.6 & $1.000 E+00$ & 8.377E-01 & $3.131 \mathrm{E}-01$ & $1.673 \mathrm{E}-04$ & $1.396 \mathrm{E}-01$ & $1.673 \mathrm{E}-07$ & $1.396 \mathrm{E}-01$ & 2.711E-05 \\
\hline 2.7 & $1.000 E+00$ & 8.325E-01 & $3.038 \mathrm{E}-01$ & $1.662 \mathrm{E}-04$ & $1.326 \mathrm{E}-01$ & $1.662 \mathrm{E}-07$ & $1.326 \mathrm{E}-01$ & 2.569E-05 \\
\hline 2.8 & $1.000 \mathrm{E}+00$ & 8.273E-01 & $2.950 \mathrm{E}-01$ & $1.652 \mathrm{E}-04$ & $1.262 \mathrm{E}-01$ & $1.652 \mathrm{E}-07$ & $1.262 \mathrm{E}-01$ & $2.436 \mathrm{E}-05$ \\
\hline 2.9 & $1.000 \mathrm{E}+00$ & $8.223 E-01$ & $2.866 \mathrm{E}-01$ & $1.642 \mathrm{E}-04$ & $1.201 \mathrm{E}-01$ & $1.642 \mathrm{E}-07$ & $1.201 \mathrm{E}-01$ & 2.313E-05 \\
\hline 3 & $1.000 \mathrm{E}+00$ & 8.172E-01 & $2.786 \mathrm{E}-01$ & $1.632 \mathrm{E}-04$ & $1.145 \mathrm{E}-01$ & $1.631 \mathrm{E}-07$ & $1.145 \mathrm{E}-01$ & 2.198E-05 \\
\hline 3.1 & $1.000 \mathrm{E}+00$ & 8.123E-01 & $2.710 \mathrm{E}-01$ & $1.622 \mathrm{E}-04$ & $1.092 \mathrm{E}-01$ & $1.622 \mathrm{E}-07$ & $1.092 \mathrm{E}-01$ & 2.090E-05 \\
\hline 3.2 & $1.000 \mathrm{E}+00$ & 8.074E-01 & $2.637 \mathrm{E}-01$ & $1.612 \mathrm{E}-04$ & $1.042 \mathrm{E}-01$ & $1.612 \mathrm{E}-07$ & $1.042 \mathrm{E}-01$ & $1.990 \mathrm{E}-05$ \\
\hline 3.3 & $1.000 \mathrm{E}+00$ & $8.026 \mathrm{E}-01$ & $2.567 \mathrm{E}-01$ & $1.602 \mathrm{E}-04$ & $9.960 \mathrm{E}-02$ & $1.602 \mathrm{E}-07$ & $9.960 \mathrm{E}-02$ & $1.896 \mathrm{E}-05$ \\
\hline 3.4 & $1.000 \mathrm{E}+00$ & $7.978 \mathrm{E}-01$ & $2.500 \mathrm{E}-01$ & $1.592 \mathrm{E}-04$ & $9.524 \mathrm{E}-02$ & $1.592 \mathrm{E}-07$ & $9.524 \mathrm{E}-02$ & $1.808 \mathrm{E}-05$ \\
\hline 3.5 & $1.000 \mathrm{E}+00$ & 7.931E-01 & $2.436 \mathrm{E}-01$ & $1.583 \mathrm{E}-04$ & $9.114 \mathrm{E}-02$ & $1.583 \mathrm{E}-07$ & $9.114 \mathrm{E}-02$ & $1.725 \mathrm{E}-05$ \\
\hline 3.6 & $1.000 \mathrm{E}+00$ & 7.884E-01 & $2.375 \mathrm{E}-01$ & $1.573 \mathrm{E}-04$ & 8.729E-02 & $1.573 \mathrm{E}-07$ & 8.729E-02 & $1.648 \mathrm{E}-05$ \\
\hline
\end{tabular}




\begin{tabular}{|c|c|c|c|c|c|c|c|c|}
\hline & & & & Activity Equi & orium Factor & & & \\
\hline $\begin{array}{l}A C H^{-1} \\
\left(h^{-1}\right)\end{array}$ & Rn-222 & Po-218 & $\mathrm{Pb}-214$ & At-218 & Bi-214 & Rn-218 & Po-214 & Tl-210 \\
\hline 3.7 & $1.000 \mathrm{E}+00$ & $7.838 \mathrm{E}-01$ & $2.316 \mathrm{E}-01$ & $1.564 \mathrm{E}-04$ & $8.366 \mathrm{E}-02$ & $1.564 \mathrm{E}-07$ & $8.366 \mathrm{E}-02$ & $1.575 \mathrm{E}-05$ \\
\hline 3.8 & $1.000 \mathrm{E}+00$ & 7.793E-01 & $2.260 \mathrm{E}-01$ & $1.555 \mathrm{E}-04$ & 8.023E-02 & $1.555 \mathrm{E}-07$ & $8.023 \mathrm{E}-02$ & $1.506 \mathrm{E}-05$ \\
\hline 3.9 & $1.000 \mathrm{E}+00$ & $7.748 \mathrm{E}-01$ & $2.205 \mathrm{E}-01$ & $1.546 \mathrm{E}-04$ & 7.700E-02 & 1.546E-07 & 7.700E-02 & $1.441 \mathrm{E}-05$ \\
\hline 4 & $1.000 \mathrm{E}+00$ & 7.703E-01 & $2.153 \mathrm{E}-01$ & $1.537 \mathrm{E}-04$ & 7.394E-02 & $1.537 \mathrm{E}-07$ & 7.394E-02 & $1.380 \mathrm{E}-05$ \\
\hline 5 & $1.000 \mathrm{E}+00$ & $7.285 \mathrm{E}-01$ & $1.725 \mathrm{E}-01$ & $1.453 \mathrm{E}-04$ & 5.090E-02 & $1.453 \mathrm{E}-07$ & $5.090 \mathrm{E}-02$ & $9.245 \mathrm{E}-06$ \\
\hline 6 & $1.000 \mathrm{E}+00$ & $6.910 \mathrm{E}-01$ & $1.420 \mathrm{E}-01$ & 1.377E-04 & 3.672E-02 & 1.377E-07 & 3.672E-02 & $6.493 \mathrm{E}-06$ \\
\hline 7 & $1.000 \mathrm{E}+00$ & $6.571 \mathrm{E}-01$ & 1.192E-01 & $1.309 \mathrm{E}-04$ & $2.745 \mathrm{E}-02$ & $1.309 \mathrm{E}-07$ & $2.745 \mathrm{E}-02$ & 4.729E-06 \\
\hline 8 & $1.000 E+00$ & $6.264 \mathrm{E}-01$ & $1.018 \mathrm{E}-01$ & $1.247 \mathrm{E}-04$ & $2.111 \mathrm{E}-02$ & $1.247 \mathrm{E}-07$ & $2.111 \mathrm{E}-02$ & $3.546 \mathrm{E}-06$ \\
\hline 9 & $1.000 \mathrm{E}+00$ & $5.985 \mathrm{E}-01$ & $8.802 \mathrm{E}-02$ & $1.191 \mathrm{E}-04$ & $1.661 \mathrm{E}-02$ & $1.190 \mathrm{E}-07$ & $1.661 \mathrm{E}-02$ & $2.722 \mathrm{E}-06$ \\
\hline 10 & $1.000 \mathrm{E}+00$ & 5.729E-01 & 7.697E-02 & $1.139 \mathrm{E}-04$ & $1.332 \mathrm{E}-02$ & $1.139 \mathrm{E}-07$ & $1.332 \mathrm{E}-02$ & $2.132 \mathrm{E}-06$ \\
\hline 15 & $1.000 E+00$ & $4.721 \mathrm{E}-01$ & $4.426 \mathrm{E}-02$ & $9.358 \mathrm{E}-05$ & $5.424 \mathrm{E}-03$ & $9.356 \mathrm{E}-08$ & $5.424 \mathrm{E}-03$ & $7.755 E-07$ \\
\hline 20 & $1.000 E+00$ & 4.015E-01 & $2.891 \mathrm{E}-02$ & 7.934E-05 & $2.742 \mathrm{E}-03$ & 7.932E-08 & 2.743E-03 & $3.544 \mathrm{E}-07$ \\
\hline 25 & $1.000 \mathrm{E}+00$ & $3.492 \mathrm{E}-01$ & $2.041 \mathrm{E}-02$ & $6.881 \mathrm{E}-05$ & $1.580 \mathrm{E}-03$ & $6.879 \mathrm{E}-08$ & $1.580 \mathrm{E}-03$ & $1.862 \mathrm{E}-07$ \\
\hline 30 & $1.000 E+00$ & $3.090 \mathrm{E}-01$ & $1.520 \mathrm{E}-02$ & $6.071 \mathrm{E}-05$ & $9.937 \mathrm{E}-04$ & $6.068 \mathrm{E}-08$ & $9.938 \mathrm{E}-04$ & $1.077 \mathrm{E}-07$ \\
\hline 35 & $1.000 \mathrm{E}+00$ & $2.771 \mathrm{E}-01$ & $1.176 \mathrm{E}-02$ & $5.428 \mathrm{E}-05$ & $6.659 \mathrm{E}-04$ & $5.425 \mathrm{E}-08$ & $6.660 \mathrm{E}-04$ & $6.678 \mathrm{E}-08$ \\
\hline 40 & $1.000 E+00$ & $2.512 \mathrm{E}-01$ & $9.380 \mathrm{E}-03$ & 4.905E-05 & 4.682E-04 & 4.902E-08 & $4.682 \mathrm{E}-04$ & 4.369E-08 \\
\hline 45 & $1.000 \mathrm{E}+00$ & 2.297E-01 & 7.656E-03 & 4.472E-05 & $3.418 \mathrm{E}-04$ & 4.469E-08 & $3.418 \mathrm{E}-04$ & $2.982 \mathrm{E}-08$ \\
\hline 50 & $1.000 E+00$ & $2.116 \mathrm{E}-01$ & $6.368 \mathrm{E}-03$ & 4.108E-05 & $2.571 \mathrm{E}-04$ & 4.105E-08 & $2.572 \mathrm{E}-04$ & 2.107E-08 \\
\hline 55 & $1.000 E+00$ & $1.961 \mathrm{E}-01$ & $5.381 \mathrm{E}-03$ & $3.796 \mathrm{E}-05$ & $1.984 \mathrm{E}-04$ & $3.793 \mathrm{E}-08$ & $1.984 \mathrm{E}-04$ & $1.532 \mathrm{E}-08$ \\
\hline 60 & $1.000 E+00$ & $1.827 \mathrm{E}-01$ & 4.607E-03 & $3.528 \mathrm{E}-05$ & $1.563 \mathrm{E}-04$ & $3.525 \mathrm{E}-08$ & $1.563 \mathrm{E}-04$ & $1.141 \mathrm{E}-08$ \\
\hline 65 & $1.000 E+00$ & $1.711 \mathrm{E}-01$ & $3.989 \mathrm{E}-03$ & $3.293 \mathrm{E}-05$ & $1.253 \mathrm{E}-04$ & $3.290 \mathrm{E}-08$ & $1.253 \mathrm{E}-04$ & 8.679E-09 \\
\hline 70 & $1.000 E+00$ & $1.608 \mathrm{E}-01$ & $3.488 \mathrm{E}-03$ & $3.087 \mathrm{E}-05$ & $1.020 \mathrm{E}-04$ & $3.084 \mathrm{E}-08$ & 1.020E-04 & $6.720 \mathrm{E}-09$ \\
\hline 75 & $1.000 E+00$ & $1.517 \mathrm{E}-01$ & $3.076 \mathrm{E}-03$ & $2.904 \mathrm{E}-05$ & $8.417 \mathrm{E}-05$ & $2.901 \mathrm{E}-08$ & $8.420 \mathrm{E}-05$ & $5.285 \mathrm{E}-09$ \\
\hline 80 & $1.000 \mathrm{E}+00$ & $1.436 \mathrm{E}-01$ & $2.733 \mathrm{E}-03$ & $2.740 \mathrm{E}-05$ & 7.027E-05 & $2.737 \mathrm{E}-08$ & 7.030E-05 & 4.215E-09 \\
\hline 85 & $1.000 E+00$ & $1.363 \mathrm{E}-01$ & $2.444 \mathrm{E}-03$ & $2.594 \mathrm{E}-05$ & 5.927E-05 & $2.591 \mathrm{E}-08$ & $5.930 \mathrm{E}-05$ & $3.404 \mathrm{E}-09$ \\
\hline 90 & $1.000 E+00$ & $1.297 \mathrm{E}-01$ & 2.199E-03 & $2.461 \mathrm{E}-05$ & $5.046 \mathrm{E}-05$ & $2.458 \mathrm{E}-08$ & $5.048 \mathrm{E}-05$ & 2.779E-09 \\
\hline 95 & $1.000 \mathrm{E}+00$ & $1.237 \mathrm{E}-01$ & $1.989 \mathrm{E}-03$ & $2.341 \mathrm{E}-05$ & 4.331E-05 & $2.338 \mathrm{E}-08$ & 4.334E-05 & 2.291E-09 \\
\hline 100 & $1.000 \mathrm{E}+00$ & $1.183 \mathrm{E}-01$ & $1.808 \mathrm{E}-03$ & $2.232 \mathrm{E}-05$ & $3.746 \mathrm{E}-05$ & $2.228 \mathrm{E}-08$ & $3.748 \mathrm{E}-05$ & 1.907E-09 \\
\hline 200 & $1.000 \mathrm{E}+00$ & $6.286 \mathrm{E}-02$ & 4.840E-04 & $1.122 \mathrm{E}-05$ & 5.121E-06 & 1.119E-08 & $5.132 \mathrm{E}-06$ & $1.483 \mathrm{E}-10$ \\
\hline 300 & $1.000 E+00$ & 4.280E-02 & 2.203E-04 & 7.253E-06 & 1.574E-06 & 7.223E-09 & $1.581 \mathrm{E}-06$ & $3.185 \mathrm{E}-11$ \\
\hline 400 & $1.000 \mathrm{E}+00$ & $3.245 \mathrm{E}-02$ & $1.254 \mathrm{E}-04$ & $5.232 \mathrm{E}-06$ & $6.790 \mathrm{E}-07$ & 5.203E-09 & $6.842 \mathrm{E}-07$ & $1.056 \mathrm{E}-11$ \\
\hline 500 & $1.000 E+00$ & $2.613 \mathrm{E}-02$ & $8.085 \mathrm{E}-05$ & $4.018 \mathrm{E}-06$ & $3.532 \mathrm{E}-07$ & $3.990 \mathrm{E}-09$ & $3.572 \mathrm{E}-07$ & $4.461 \mathrm{E}-12$ \\
\hline 600 & $1.000 \mathrm{E}+00$ & $2.187 \mathrm{E}-02$ & $5.642 \mathrm{E}-05$ & $3.215 \mathrm{E}-06$ & $2.070 \mathrm{E}-07$ & $3.188 \mathrm{E}-09$ & $2.102 \mathrm{E}-07$ & $2.200 \mathrm{E}-12$ \\
\hline 700 & $1.000 E+00$ & $1.880 \mathrm{E}-02$ & 4.160E-05 & $2.647 \mathrm{E}-06$ & $1.317 \mathrm{E}-07$ & $2.621 \mathrm{E}-09$ & $1.343 \mathrm{E}-07$ & 1.209E-12 \\
\hline 800 & $1.000 \mathrm{E}+00$ & $1.649 \mathrm{E}-02$ & 3.193E-05 & 2.227E-06 & $8.900 \mathrm{E}-08$ & 2.203E-09 & $9.119 \mathrm{E}-08$ & $7.186 \mathrm{E}-13$ \\
\hline 900 & $1.000 E+00$ & $1.469 \mathrm{E}-02$ & $2.528 \mathrm{E}-05$ & $1.906 \mathrm{E}-06$ & $6.298 \mathrm{E}-08$ & $1.882 \mathrm{E}-09$ & $6.486 \mathrm{E}-08$ & $4.540 \mathrm{E}-13$ \\
\hline
\end{tabular}

${ }^{a} 0.18$ is the residential default $\mathrm{ACH}$, and 0.6 is the commercial/industrial default $\mathrm{ACH}$. 


\section{APPENDIX B. INHALATION FRACTIONAL EQUILIBRIUM FACTORS BASED ON AIR EXCHANGE RATE}



Table B-1: Actinon Inhalation Fractional Equilibrium Factors Based on Air Changes Per Hour

\begin{tabular}{|c|c|c|c|c|c|c|c|}
\hline $\mathbf{A C H}^{\mathbf{a}}$ & Rn-219 & Po-215 & Pb-211 & Bi-211 & Tl-207 & Po-211 & FEQ \\
\hline 0 & $1.000 \mathrm{E}+00$ & $9.972 \mathrm{E}-01$ & $1.000 \mathrm{E}+00$ & $1.000 \mathrm{E}+00$ & $9.970 \mathrm{E}-01$ & $2.800 \mathrm{E}-03$ & $1.000 \mathrm{E}+00$ \\
\hline 0.1 & $1.000 \mathrm{E}+00$ & $9.968 \mathrm{E}-01$ & $9.201 \mathrm{E}-01$ & $9.154 \mathrm{E}-01$ & $9.023 \mathrm{E}-01$ & $2.500 \mathrm{E}-03$ & $9.153 \mathrm{E}-01$ \\
\hline 0.18 & $1.000 \mathrm{E}+00$ & $1.000 \mathrm{E}+00$ & $8.648 \mathrm{E}-01$ & $8.569 \mathrm{E}-01$ & 8.371E-01 & $2.400 \mathrm{E}-03$ & 8.569E-01 \\
\hline 0.2 & $1.000 \mathrm{E}+00$ & $9.964 \mathrm{E}-01$ & $8.521 \mathrm{E}-01$ & $8.434 \mathrm{E}-01$ & $8.220 \mathrm{E}-01$ & $2.300 \mathrm{E}-03$ & $8.433 \mathrm{E}-01$ \\
\hline 0.3 & $1.000 \mathrm{E}+00$ & $9.960 \mathrm{E}-01$ & 7.934E-01 & 7.813E-01 & 7.530E-01 & $2.200 \mathrm{E}-03$ & $7.813 \mathrm{E}-01$ \\
\hline 0.4 & $1.000 \mathrm{E}+00$ & $9.956 \mathrm{E}-01$ & 7.422E-01 & 7.273E-01 & $6.933 \mathrm{E}-01$ & $2.000 \mathrm{E}-03$ & 7.273E-01 \\
\hline 0.5 & $1.000 \mathrm{E}+00$ & $9.951 \mathrm{E}-01$ & $6.973 \mathrm{E}-01$ & $6.798 \mathrm{E}-01$ & $6.410 \mathrm{E}-01$ & $1.900 \mathrm{E}-03$ & 6.799E-01 \\
\hline 0.6 & $1.000 \mathrm{E}+00$ & $9.946 \mathrm{E}-01$ & $6.575 \mathrm{E}-01$ & $6.378 \mathrm{E}-01$ & $5.949 \mathrm{E}-01$ & $1.800 \mathrm{E}-03$ & $6.379 \mathrm{E}-01$ \\
\hline 0.7 & $1.000 \mathrm{E}+00$ & $9.904 \mathrm{E}-01$ & $6.220 \mathrm{E}-01$ & $6.004 \mathrm{E}-01$ & $5.541 \mathrm{E}-01$ & $1.700 \mathrm{E}-03$ & $6.004 \mathrm{E}-01$ \\
\hline 0.8 & $1.000 \mathrm{E}+00$ & 9.917E-01 & 5.901E-01 & $5.668 \mathrm{E}-01$ & $5.176 \mathrm{E}-01$ & $1.600 \mathrm{E}-03$ & $5.668 \mathrm{E}-01$ \\
\hline 0.9 & $1.000 \mathrm{E}+00$ & $9.929 \mathrm{E}-01$ & $5.613 \mathrm{E}-01$ & $5.365 \mathrm{E}-01$ & $4.848 \mathrm{E}-01$ & $1.500 \mathrm{E}-03$ & $5.366 \mathrm{E}-01$ \\
\hline 1 & $1.000 \mathrm{E}+00$ & $9.940 \mathrm{E}-01$ & $5.352 \mathrm{E}-01$ & $5.091 \mathrm{E}-01$ & $4.553 \mathrm{E}-01$ & $1.400 \mathrm{E}-03$ & 5.091E-01 \\
\hline 1.1 & $1.000 \mathrm{E}+00$ & $9.950 \mathrm{E}-01$ & $5.115 \mathrm{E}-01$ & $4.841 \mathrm{E}-01$ & $4.286 \mathrm{E}-01$ & $1.300 \mathrm{E}-03$ & $4.841 \mathrm{E}-01$ \\
\hline 1.2 & $1.000 \mathrm{E}+00$ & $9.959 E-01$ & 4.897E-01 & $4.612 \mathrm{E}-01$ & $4.042 \mathrm{E}-01$ & $1.300 \mathrm{E}-03$ & 4.613E-01 \\
\hline 1.3 & $1.000 \mathrm{E}+00$ & $9.967 \mathrm{E}-01$ & 4.697E-01 & 4.403E-01 & $3.820 \mathrm{E}-01$ & $1.200 \mathrm{E}-03$ & 4.404E-01 \\
\hline 1.4 & $1.000 E+00$ & $9.974 \mathrm{E}-01$ & 4.513E-01 & 4.210E-01 & 3.617E-01 & $1.200 \mathrm{E}-03$ & 4.211E-01 \\
\hline 1.5 & $1.000 \mathrm{E}+00$ & $9.981 \mathrm{E}-01$ & 4.343E-01 & $4.032 \mathrm{E}-01$ & $3.430 \mathrm{E}-01$ & $1.100 \mathrm{E}-03$ & $4.033 \mathrm{E}-01$ \\
\hline 1.6 & $1.000 \mathrm{E}+00$ & $9.987 \mathrm{E}-01$ & 4.185E-01 & $3.867 \mathrm{E}-01$ & $3.257 \mathrm{E}-01$ & $1.100 \mathrm{E}-03$ & $3.868 \mathrm{E}-01$ \\
\hline 1.7 & $1.000 \mathrm{E}+00$ & $9.992 \mathrm{E}-01$ & $4.038 \mathrm{E}-01$ & $3.714 \mathrm{E}-01$ & $3.098 \mathrm{E}-01$ & $1.000 \mathrm{E}-03$ & $3.714 \mathrm{E}-01$ \\
\hline 1.8 & $1.000 \mathrm{E}+00$ & 9.997E-01 & $3.902 \mathrm{E}-01$ & $3.571 \mathrm{E}-01$ & $2.951 \mathrm{E}-01$ & $1.000 \mathrm{E}-03$ & $3.572 \mathrm{E}-01$ \\
\hline 1.9 & $1.000 \mathrm{E}+00$ & $1.000 E+00$ & 3.774E-01 & 3.438E-01 & $2.814 \mathrm{E}-01$ & $9.000 \mathrm{E}-04$ & 3.437E-01 \\
\hline 2 & $1.000 \mathrm{E}+00$ & $1.000 \mathrm{E}+00$ & $3.654 \mathrm{E}-01$ & 3.313E-01 & 2.687E-01 & $9.000 \mathrm{E}-04$ & 3.314E-01 \\
\hline 2.1 & $1.000 \mathrm{E}+00$ & $1.000 E+00$ & $3.541 \mathrm{E}-01$ & 3.196E-01 & $2.568 \mathrm{E}-01$ & $9.000 \mathrm{E}-04$ & 3.197E-01 \\
\hline 2.2 & $1.000 \mathrm{E}+00$ & $1.000 \mathrm{E}+00$ & $3.436 \mathrm{E}-01$ & $3.086 \mathrm{E}-01$ & $2.457 \mathrm{E}-01$ & $9.000 \mathrm{E}-04$ & $3.087 \mathrm{E}-01$ \\
\hline 2.3 & $1.000 \mathrm{E}+00$ & $1.000 \mathrm{E}+00$ & $3.336 \mathrm{E}-01$ & 2.983E-01 & $2.353 \mathrm{E}-01$ & $8.000 \mathrm{E}-04$ & $2.984 \mathrm{E}-01$ \\
\hline 2.4 & $1.000 \mathrm{E}+00$ & $1.000 \mathrm{E}+00$ & $3.242 \mathrm{E}-01$ & 2.886E-01 & $2.256 \mathrm{E}-01$ & $8.000 \mathrm{E}-04$ & $2.887 \mathrm{E}-01$ \\
\hline 2.5 & $1.000 \mathrm{E}+00$ & $1.000 \mathrm{E}+00$ & $3.153 \mathrm{E}-01$ & $2.794 \mathrm{E}-01$ & $2.165 \mathrm{E}-01$ & $8.000 \mathrm{E}-04$ & $2.795 \mathrm{E}-01$ \\
\hline 2.6 & $1.000 \mathrm{E}+00$ & $1.000 E+00$ & $3.069 \mathrm{E}-01$ & 2.707E-01 & 2.079E-01 & 7.000E-04 & 2.707E-01 \\
\hline 2.7 & $1.000 \mathrm{E}+00$ & $1.000 \mathrm{E}+00$ & $2.990 \mathrm{E}-01$ & 2.625E-01 & $1.998 \mathrm{E}-01$ & 7.000E-04 & $2.626 \mathrm{E}-01$ \\
\hline 2.8 & $1.000 \mathrm{E}+00$ & $1.000 \mathrm{E}+00$ & $2.914 \mathrm{E}-01$ & $2.547 \mathrm{E}-01$ & $1.922 \mathrm{E}-01$ & 7.000E-04 & $2.548 \mathrm{E}-01$ \\
\hline 2.9 & $1.000 E+00$ & $1.000 E+00$ & $2.842 \mathrm{E}-01$ & $2.473 \mathrm{E}-01$ & $1.850 \mathrm{E}-01$ & 7.000E-04 & $2.474 \mathrm{E}-01$ \\
\hline 3 & $1.000 \mathrm{E}+00$ & $1.000 \mathrm{E}+00$ & $2.773 \mathrm{E}-01$ & $2.403 \mathrm{E}-01$ & $1.782 \mathrm{E}-01$ & $7.000 \mathrm{E}-04$ & $2.404 \mathrm{E}-01$ \\
\hline 3.1 & $1.000 \mathrm{E}+00$ & $1.000 E+00$ & $2.708 \mathrm{E}-01$ & $2.336 \mathrm{E}-01$ & $1.718 \mathrm{E}-01$ & $6.000 \mathrm{E}-04$ & $2.336 \mathrm{E}-01$ \\
\hline 3.2 & $1.000 \mathrm{E}+00$ & $1.000 \mathrm{E}+00$ & $2.646 \mathrm{E}-01$ & $2.272 \mathrm{E}-01$ & $1.657 \mathrm{E}-01$ & $6.000 \mathrm{E}-04$ & $2.273 \mathrm{E}-01$ \\
\hline 3.3 & $1.000 \mathrm{E}+00$ & $1.000 \mathrm{E}+00$ & $2.586 \mathrm{E}-01$ & $2.211 \mathrm{E}-01$ & $1.599 \mathrm{E}-01$ & $6.000 \mathrm{E}-04$ & $2.212 \mathrm{E}-01$ \\
\hline 3.4 & $1.000 E+00$ & $1.000 E+00$ & $2.530 \mathrm{E}-01$ & $2.153 \mathrm{E}-01$ & $1.544 \mathrm{E}-01$ & $6.000 \mathrm{E}-04$ & $2.154 \mathrm{E}-01$ \\
\hline 3.5 & $1.000 \mathrm{E}+00$ & $1.000 E+00$ & $2.475 \mathrm{E}-01$ & 2.097E-01 & $1.492 \mathrm{E}-01$ & $6.000 \mathrm{E}-04$ & $2.098 \mathrm{E}-01$ \\
\hline 3.6 & $1.000 \mathrm{E}+00$ & $1.000 \mathrm{E}+00$ & $2.423 \mathrm{E}-01$ & $2.044 \mathrm{E}-01$ & $1.443 \mathrm{E}-01$ & $6.000 \mathrm{E}-04$ & $2.045 \mathrm{E}-01$ \\
\hline 3.7 & $1.000 \mathrm{E}+00$ & $1.000 \mathrm{E}+00$ & $2.373 \mathrm{E}-01$ & $1.994 \mathrm{E}-01$ & 1.395E-01 & $5.000 \mathrm{E}-04$ & $1.995 \mathrm{E}-01$ \\
\hline
\end{tabular}




\begin{tabular}{|c|c|c|c|c|c|c|c|}
\hline $\mathbf{A C H}^{\mathbf{a}}$ & Rn-219 & Po-215 & $\mathrm{Pb}-211$ & Bi-211 & Tl-207 & Po-211 & FEQ \\
\hline 3.8 & $1.000 E+00$ & $1.000 E+00$ & $2.325 \mathrm{E}-01$ & $1.945 \mathrm{E}-01$ & 1.350E-01 & $5.000 \mathrm{E}-04$ & 1.945E-01 \\
\hline 3.9 & $1.000 E+00$ & $1.000 \mathrm{E}+00$ & 2.279E-01 & $1.898 \mathrm{E}-01$ & $1.308 \mathrm{E}-01$ & $5.000 \mathrm{E}-04$ & 1.899E-01 \\
\hline 4 & $1.000 \mathrm{E}+00$ & $1.000 \mathrm{E}+00$ & $2.235 \mathrm{E}-01$ & $1.853 \mathrm{E}-01$ & 1.267E-01 & $5.000 \mathrm{E}-04$ & $1.854 \mathrm{E}-01$ \\
\hline 5 & $1.000 \mathrm{E}+00$ & 9.967E-01 & $1.871 \mathrm{E}-01$ & $1.488 \mathrm{E}-01$ & $9.430 \mathrm{E}-02$ & $4.000 \mathrm{E}-04$ & $1.489 \mathrm{E}-01$ \\
\hline 6 & $1.000 \mathrm{E}+00$ & $9.928 \mathrm{E}-01$ & 1.609E-01 & $1.230 \mathrm{E}-01$ & 7.260E-02 & $3.000 \mathrm{E}-04$ & $1.230 \mathrm{E}-01$ \\
\hline 7 & $1.000 \mathrm{E}+00$ & 9.997E-01 & $1.412 \mathrm{E}-01$ & $1.038 \mathrm{E}-01$ & $5.740 \mathrm{E}-02$ & $3.000 \mathrm{E}-04$ & $1.039 \mathrm{E}-01$ \\
\hline 8 & $1.000 \mathrm{E}+00$ & $1.000 \mathrm{E}+00$ & $1.257 \mathrm{E}-01$ & $8.910 \mathrm{E}-02$ & $4.630 \mathrm{E}-02$ & 2.000E-04 & 8.914E-02 \\
\hline 9 & $1.000 E+00$ & $1.000 E+00$ & $1.133 \mathrm{E}-01$ & 7.750E-02 & $3.800 \mathrm{E}-02$ & $2.000 \mathrm{E}-04$ & 7.758E-02 \\
\hline 10 & $1.000 \mathrm{E}+00$ & $9.961 \mathrm{E}-01$ & $1.032 \mathrm{E}-01$ & $6.810 \mathrm{E}-02$ & $3.160 \mathrm{E}-02$ & 2.000E-04 & $6.821 \mathrm{E}-02$ \\
\hline 15 & $1.000 \mathrm{E}+00$ & $9.954 \mathrm{E}-01$ & 7.120E-02 & 4.020E-02 & $1.470 \mathrm{E}-02$ & $1.000 \mathrm{E}-04$ & 4.029E-02 \\
\hline 20 & $1.000 \mathrm{E}+00$ & 9.947E-01 & $5.430 \mathrm{E}-02$ & $2.680 \mathrm{E}-02$ & $8.100 \mathrm{E}-03$ & $1.000 \mathrm{E}-04$ & $2.683 \mathrm{E}-02$ \\
\hline 25 & $1.000 E+00$ & 9.939E-01 & 4.390E-02 & $1.920 \mathrm{E}-02$ & 4.900E-03 & 1.000E-04 & $1.935 \mathrm{E}-02$ \\
\hline 30 & $1.000 E+00$ & $9.932 \mathrm{E}-01$ & $3.680 \mathrm{E}-02$ & $1.450 \mathrm{E}-02$ & $3.200 \mathrm{E}-03$ & $0.000 E+00$ & $1.445 \mathrm{E}-02$ \\
\hline 35 & $1.000 E+00$ & $9.923 \mathrm{E}-01$ & $3.170 \mathrm{E}-02$ & $1.130 \mathrm{E}-02$ & 2.300E-03 & $0.000 E+00$ & $1.136 \mathrm{E}-02$ \\
\hline 40 & $1.000 E+00$ & $9.915 \mathrm{E}-01$ & $2.780 \mathrm{E}-02$ & $9.100 \mathrm{E}-03$ & 1.600E-03 & $0.000 E+00$ & 9.171E-03 \\
\hline 45 & $1.000 \mathrm{E}+00$ & $9.906 \mathrm{E}-01$ & $2.480 \mathrm{E}-02$ & 7.500E-03 & 1.200E-03 & $0.000 E+00$ & $7.476 \mathrm{E}-03$ \\
\hline 50 & $1.000 \mathrm{E}+00$ & $9.897 \mathrm{E}-01$ & $2.240 \mathrm{E}-02$ & $6.300 \mathrm{E}-03$ & $9.000 \mathrm{E}-04$ & $0.000 E+00$ & $6.280 \mathrm{E}-03$ \\
\hline 55 & $1.000 \mathrm{E}+00$ & $9.888 \mathrm{E}-01$ & 2.040E-02 & $5.300 \mathrm{E}-03$ & 7.000E-04 & $0.000 E+00$ & 5.383E-03 \\
\hline 60 & $1.000 E+00$ & $9.878 \mathrm{E}-01$ & $1.870 \mathrm{E}-02$ & 4.600E-03 & $6.000 \mathrm{E}-04$ & $0.000 E+00$ & 4.585E-03 \\
\hline 65 & $1.000 E+00$ & $9.868 \mathrm{E}-01$ & $1.730 \mathrm{E}-02$ & 4.000E-03 & $5.000 \mathrm{E}-04$ & $0.000 E+00$ & 3.987E-03 \\
\hline 70 & $1.000 \mathrm{E}+00$ & $9.891 \mathrm{E}-01$ & $1.600 \mathrm{E}-02$ & $3.500 \mathrm{E}-03$ & 4.000E-04 & $0.000 E+00$ & $3.489 \mathrm{E}-03$ \\
\hline 75 & $1.000 \mathrm{E}+00$ & $9.882 \mathrm{E}-01$ & $1.500 \mathrm{E}-02$ & 3.100E-03 & $3.000 \mathrm{E}-04$ & $0.000 E+00$ & $3.090 \mathrm{E}-03$ \\
\hline 80 & $1.000 E+00$ & $9.873 \mathrm{E}-01$ & $1.400 \mathrm{E}-02$ & 2.700E-03 & $3.000 \mathrm{E}-04$ & $0.000 E+00$ & 2.791E-03 \\
\hline 85 & $1.000 E+00$ & $9.864 \mathrm{E}-01$ & 1.320E-02 & $2.500 \mathrm{E}-03$ & $2.000 \mathrm{E}-04$ & $0.000 E+00$ & $2.492 \mathrm{E}-03$ \\
\hline 90 & $1.000 \mathrm{E}+00$ & $9.854 \mathrm{E}-01$ & $1.250 \mathrm{E}-02$ & $2.200 \mathrm{E}-03$ & $2.000 \mathrm{E}-04$ & $0.000 E+00$ & 2.193E-03 \\
\hline 95 & $1.000 \mathrm{E}+00$ & $9.844 \mathrm{E}-01$ & $1.180 \mathrm{E}-02$ & 2.000E-03 & 2.000E-04 & $0.000 E+00$ & $1.994 \mathrm{E}-03$ \\
\hline 100 & $1.000 E+00$ & $9.833 \mathrm{E}-01$ & 1.120E-02 & $1.800 \mathrm{E}-03$ & $1.000 \mathrm{E}-04$ & $0.000 E+00$ & $1.894 \mathrm{E}-03$ \\
\hline 200 & $1.000 \mathrm{E}+00$ & $9.730 \mathrm{E}-01$ & $5.600 \mathrm{E}-03$ & $5.000 \mathrm{E}-04$ & $0.000 E+00$ & $0.000 E+00$ & 4.984E-04 \\
\hline 300 & $1.000 \mathrm{E}+00$ & $9.629 \mathrm{E}-01$ & $3.700 \mathrm{E}-03$ & $2.000 \mathrm{E}-04$ & $0.000 E+00$ & $0.000 E+00$ & $1.994 \mathrm{E}-04$ \\
\hline 400 & $1.000 E+00$ & $9.464 \mathrm{E}-01$ & 2.700E-03 & $1.000 \mathrm{E}-04$ & $0.000 E+00$ & $0.000 E+00$ & $9.968 \mathrm{E}-05$ \\
\hline 500 & $1.000 E+00$ & $9.277 \mathrm{E}-01$ & 2.100E-03 & $1.000 \mathrm{E}-04$ & $0.000 E+00$ & $0.000 E+00$ & $9.968 \mathrm{E}-05$ \\
\hline 600 & $1.000 E+00$ & $9.224 \mathrm{E}-01$ & $1.800 \mathrm{E}-03$ & $1.000 \mathrm{E}-04$ & $0.000 E+00$ & $0.000 E+00$ & $9.968 \mathrm{E}-05$ \\
\hline 700 & $1.000 \mathrm{E}+00$ & $9.078 \mathrm{E}-01$ & $1.500 \mathrm{E}-03$ & $0.000 E+00$ & $0.000 E+00$ & $0.000 E+00$ & $0.000 E+00$ \\
\hline 800 & $1.000 \mathrm{E}+00$ & $8.951 \mathrm{E}-01$ & $1.300 \mathrm{E}-03$ & $0.000 E+00$ & $0.000 E+00$ & $0.000 E+00$ & $0.000 E+00$ \\
\hline 900 & $1.000 E+00$ & 8.831E-01 & 1.100E-03 & $0.000 E+00$ & $0.000 E+00$ & $0.000 E+00$ & $0.000 E+00$ \\
\hline
\end{tabular}

${ }^{\mathrm{a}} 0.18$ is the residential default $\mathrm{ACH}$, and 0.6 is the commercial/industrial default $\mathrm{ACH}$. 
Table B-2: Thoron Inhalation Fractional Equilibrium Factors Based on Air Changes Per Hour

\begin{tabular}{|c|c|c|c|c|c|c|c|}
\hline $\mathbf{A C H}^{\mathrm{a}}$ & Rn-220 & Po-216 & Pb-212 & Bi-212 & Po-212 & Tl-208 & FEQ \\
\hline 0 & $1.000 E+00$ & $1.000 E+00$ & $1.000 E+00$ & $1.000 E+00$ & $6.410 \mathrm{E}-01$ & 3.590E-01 & $1.000 E+00$ \\
\hline 0.1 & $1.000 E+00$ & $1.000 E+00$ & $3.945 \mathrm{E}-01$ & $3.443 \mathrm{E}-01$ & 2.207E-01 & 1.227E-01 & $3.443 E-01$ \\
\hline 0.18 & $1.000 \mathrm{E}+00$ & $1.000 E+00$ & 2.657E-01 & 2.106E-01 & $1.350 \mathrm{E}-01$ & 7.460E-02 & 2.106E-01 \\
\hline 0.2 & $1.000 E+00$ & $1.000 E+00$ & $2.457 \mathrm{E}-01$ & $1.903 \mathrm{E}-01$ & $1.220 \mathrm{E}-01$ & $6.730 \mathrm{E}-02$ & $1.903 \mathrm{E}-01$ \\
\hline 0.3 & $1.000 \mathrm{E}+00$ & $1.000 \mathrm{E}+00$ & $1.784 \mathrm{E}-01$ & $1.242 \mathrm{E}-01$ & 7.960E-02 & $4.360 \mathrm{E}-02$ & $1.242 \mathrm{E}-01$ \\
\hline 0.4 & $1.000 E+00$ & $1.000 \mathrm{E}+00$ & $1.401 \mathrm{E}-01$ & $8.850 \mathrm{E}-02$ & $5.670 \mathrm{E}-02$ & $3.090 \mathrm{E}-02$ & 8.847E-02 \\
\hline 0.5 & $1.000 E+00$ & $1.000 E+00$ & 1.153E-01 & $6.670 \mathrm{E}-02$ & $4.280 \mathrm{E}-02$ & $2.310 \mathrm{E}-02$ & $6.675 \mathrm{E}-02$ \\
\hline 0.6 & $1.000 E+00$ & $1.000 E+00$ & $9.790 \mathrm{E}-02$ & $5.230 \mathrm{E}-02$ & 3.350E-02 & $1.800 \mathrm{E}-02$ & 5.227E-02 \\
\hline 0.7 & $1.000 \mathrm{E}+00$ & $1.000 E+00$ & $8.510 \mathrm{E}-02$ & 4.220E-02 & 2.700E-02 & $1.440 \mathrm{E}-02$ & $4.214 \mathrm{E}-02$ \\
\hline 0.8 & $1.000 E+00$ & $1.000 E+00$ & 7.530E-02 & $3.480 \mathrm{E}-02$ & $2.230 \mathrm{E}-02$ & $1.180 \mathrm{E}-02$ & $3.479 E-02$ \\
\hline 0.9 & $1.000 E+00$ & 9.999E-01 & $6.750 \mathrm{E}-02$ & 2.920E-02 & $1.870 \mathrm{E}-02$ & $9.800 \mathrm{E}-03$ & $2.918 \mathrm{E}-02$ \\
\hline 1 & $1.000 E+00$ & 9.999E-01 & $6.120 \mathrm{E}-02$ & $2.490 \mathrm{E}-02$ & $1.600 \mathrm{E}-02$ & 8.300E-03 & 2.494E-02 \\
\hline 1.1 & $1.000 E+00$ & 9.999E-01 & $5.590 \mathrm{E}-02$ & $2.150 \mathrm{E}-02$ & $1.380 \mathrm{E}-02$ & 7.100E-03 & $2.152 \mathrm{E}-02$ \\
\hline 1.2 & $1.000 \mathrm{E}+00$ & 9.999E-01 & $5.150 \mathrm{E}-02$ & $1.870 \mathrm{E}-02$ & $1.200 \mathrm{E}-02$ & $6.200 \mathrm{E}-03$ & $1.871 \mathrm{E}-02$ \\
\hline 1.3 & $1.000 E+00$ & $9.999 \mathrm{E}-01$ & 4.770E-02 & $1.650 \mathrm{E}-02$ & $1.060 \mathrm{E}-02$ & $5.400 \mathrm{E}-03$ & $1.653 \mathrm{E}-02$ \\
\hline 1.4 & $1.000 E+00$ & 9.999E-01 & 4.450E-02 & $1.460 \mathrm{E}-02$ & $9.400 \mathrm{E}-03$ & 4.800E-03 & $1.465 \mathrm{E}-02$ \\
\hline 1.5 & $1.000 E+00$ & 9.999E-01 & 4.160E-02 & 1.310E-02 & 8.400E-03 & 4.200E-03 & $1.310 \mathrm{E}-02$ \\
\hline 1.6 & $1.000 E+00$ & 9.999E-01 & 3.910E-02 & $1.170 \mathrm{E}-02$ & 7.500E-03 & 3.800E-03 & 1.170E-02 \\
\hline 1.7 & $1.000 E+00$ & 9.999E-01 & $3.690 \mathrm{E}-02$ & $1.060 \mathrm{E}-02$ & $6.800 \mathrm{E}-03$ & $3.400 \mathrm{E}-03$ & $1.061 \mathrm{E}-02$ \\
\hline 1.8 & $1.000 E+00$ & 9.999E-01 & $3.490 \mathrm{E}-02$ & $9.600 \mathrm{E}-03$ & $6.200 \mathrm{E}-03$ & 3.100E-03 & $9.652 \mathrm{E}-03$ \\
\hline 1.9 & $1.000 E+00$ & $9.999 \mathrm{E}-01$ & $3.310 \mathrm{E}-02$ & $8.800 \mathrm{E}-03$ & $5.600 \mathrm{E}-03$ & $2.800 \mathrm{E}-03$ & 8.754E-03 \\
\hline 2 & $1.000 E+00$ & $9.999 E-01$ & $3.150 \mathrm{E}-02$ & $8.100 \mathrm{E}-03$ & $5.200 \mathrm{E}-03$ & $2.500 \mathrm{E}-03$ & 8.109E-03 \\
\hline 2.1 & $1.000 E+00$ & $9.999 \mathrm{E}-01$ & 3.010E-02 & 7.400E-03 & 4.800E-03 & $2.300 \mathrm{E}-03$ & 7.464E-03 \\
\hline 2.2 & $1.000 E+00$ & $9.999 \mathrm{E}-01$ & $2.880 \mathrm{E}-02$ & $6.800 \mathrm{E}-03$ & 4.400E-03 & $2.100 \mathrm{E}-03$ & $6.846 \mathrm{E}-03$ \\
\hline 2.3 & $1.000 E+00$ & 9.999E-01 & $2.750 \mathrm{E}-02$ & $6.300 \mathrm{E}-03$ & 4.100E-03 & 1.900E-03 & $6.369 \mathrm{E}-03$ \\
\hline 2.4 & $1.000 E+00$ & $9.999 \mathrm{E}-01$ & $2.640 \mathrm{E}-02$ & $5.900 \mathrm{E}-03$ & $3.800 \mathrm{E}-03$ & $1.800 \mathrm{E}-03$ & 5.920E-03 \\
\hline 2.5 & $1.000 \mathrm{E}+00$ & $9.999 \mathrm{E}-01$ & $2.540 \mathrm{E}-02$ & 5.500E-03 & $3.500 \mathrm{E}-03$ & 1.700E-03 & $5.471 \mathrm{E}-03$ \\
\hline 2.6 & $1.000 E+00$ & $9.998 \mathrm{E}-01$ & $2.440 \mathrm{E}-02$ & $5.100 \mathrm{E}-03$ & 3.300E-03 & $1.500 \mathrm{E}-03$ & $5.135 \mathrm{E}-03$ \\
\hline 2.7 & $1.000 E+00$ & $9.998 \mathrm{E}-01$ & $2.360 \mathrm{E}-02$ & 4.800E-03 & 3.100E-03 & 1.400E-03 & 4.826E-03 \\
\hline 2.8 & $1.000 E+00$ & $9.998 \mathrm{E}-01$ & $2.270 \mathrm{E}-02$ & 4.500E-03 & $2.900 \mathrm{E}-03$ & 1.300E-03 & 4.517E-03 \\
\hline 2.9 & $1.000 E+00$ & $9.998 \mathrm{E}-01$ & $2.200 \mathrm{E}-02$ & 4.200E-03 & $2.700 \mathrm{E}-03$ & 1.200E-03 & 4.209E-03 \\
\hline 3 & $1.000 E+00$ & $9.998 \mathrm{E}-01$ & $2.120 \mathrm{E}-02$ & 4.000E-03 & $2.500 \mathrm{E}-03$ & 1.200E-03 & $3.928 \mathrm{E}-03$ \\
\hline 3.1 & $1.000 E+00$ & $9.998 \mathrm{E}-01$ & $2.060 \mathrm{E}-02$ & 3.700E-03 & $2.400 \mathrm{E}-03$ & 1.100E-03 & $3.732 \mathrm{E}-03$ \\
\hline 3.2 & $1.000 E+00$ & $9.998 \mathrm{E}-01$ & 1.990E-02 & 3.500E-03 & $2.300 \mathrm{E}-03$ & 1.000E-03 & $3.564 \mathrm{E}-03$ \\
\hline 3.3 & $1.000 E+00$ & $9.998 \mathrm{E}-01$ & $1.940 \mathrm{E}-02$ & $3.300 \mathrm{E}-03$ & $2.100 \mathrm{E}-03$ & 1.000E-03 & $3.283 \mathrm{E}-03$ \\
\hline 3.4 & $1.000 E+00$ & $9.998 \mathrm{E}-01$ & $1.880 \mathrm{E}-02$ & $3.200 \mathrm{E}-03$ & $2.000 \mathrm{E}-03$ & $9.000 \mathrm{E}-04$ & $3.142 \mathrm{E}-03$ \\
\hline 3.5 & $1.000 E+00$ & $9.998 \mathrm{E}-01$ & $1.830 \mathrm{E}-02$ & $3.000 \mathrm{E}-03$ & $1.900 \mathrm{E}-03$ & $9.000 \mathrm{E}-04$ & $2.974 \mathrm{E}-03$ \\
\hline 3.6 & $1.000 E+00$ & $9.998 \mathrm{E}-01$ & $1.780 \mathrm{E}-02$ & $2.800 \mathrm{E}-03$ & $1.800 \mathrm{E}-03$ & $8.000 \mathrm{E}-04$ & $2.806 \mathrm{E}-03$ \\
\hline 3.7 & $1.000 E+00$ & $9.998 \mathrm{E}-01$ & $1.730 \mathrm{E}-02$ & 2.700E-03 & $1.700 \mathrm{E}-03$ & 8.000E-04 & $2.665 \mathrm{E}-03$ \\
\hline
\end{tabular}




\begin{tabular}{|c|c|c|c|c|c|c|c|}
\hline $\mathbf{A C H}^{\mathbf{a}}$ & Rn-220 & Po-216 & $\mathrm{Pb}-212$ & Bi-212 & Po-212 & Tl-208 & FEQ \\
\hline 3.8 & $1.000 E+00$ & $9.998 \mathrm{E}-01$ & 1.690E-02 & 2.600E-03 & 1.700E-03 & 7.000E-04 & $2.638 \mathrm{E}-03$ \\
\hline 3.9 & $1.000 E+00$ & $9.998 \mathrm{E}-01$ & $1.640 \mathrm{E}-02$ & $2.500 \mathrm{E}-03$ & 1.600E-03 & 7.000E-04 & 2.497E-03 \\
\hline 4 & $1.000 E+00$ & $9.998 \mathrm{E}-01$ & $1.600 \mathrm{E}-02$ & $2.300 \mathrm{E}-03$ & $1.500 \mathrm{E}-03$ & 7.000E-04 & 2.329E-03 \\
\hline 5 & $1.000 E+00$ & 9.997E-01 & 1.290E-02 & 1.600E-03 & 1.000E-03 & 4.000E-04 & $1.571 \mathrm{E}-03$ \\
\hline 6 & $1.000 E+00$ & 9.997E-01 & 1.070E-02 & 1.100E-03 & 7.000E-04 & 3.000E-04 & 1.094E-03 \\
\hline 7 & $1.000 \mathrm{E}+00$ & $9.996 \mathrm{E}-01$ & $9.200 \mathrm{E}-03$ & 8.000E-04 & $5.000 \mathrm{E}-04$ & $2.000 \mathrm{E}-04$ & 7.856E-04 \\
\hline 8 & $1.000 \mathrm{E}+00$ & $9.995 \mathrm{E}-01$ & $8.100 \mathrm{E}-03$ & $6.000 \mathrm{E}-04$ & 4.000E-04 & $1.000 \mathrm{E}-04$ & $6.173 E-04$ \\
\hline 9 & $1.000 E+00$ & $9.995 \mathrm{E}-01$ & 7.200E-03 & $5.000 \mathrm{E}-04$ & $3.000 \mathrm{E}-04$ & 1.000E-04 & 4.769E-04 \\
\hline 10 & $1.000 \mathrm{E}+00$ & 9.994E-01 & $6.500 \mathrm{E}-03$ & 4.000E-04 & $3.000 \mathrm{E}-04$ & 1.000E-04 & 4.491E-04 \\
\hline 15 & $1.000 \mathrm{E}+00$ & 9.991E-01 & 4.300E-03 & $2.000 \mathrm{E}-04$ & $1.000 \mathrm{E}-04$ & $0.000 E+00$ & $1.683 \mathrm{E}-04$ \\
\hline 20 & $1.000 \mathrm{E}+00$ & $9.988 \mathrm{E}-01$ & $3.200 \mathrm{E}-03$ & $1.000 \mathrm{E}-04$ & 1.000E-04 & $0.000 E+00$ & 1.404E-04 \\
\hline 25 & $1.000 E+00$ & $9.985 \mathrm{E}-01$ & 2.600E-03 & 1.000E-04 & $0.000 E+00$ & $0.000 E+00$ & 2.786E-05 \\
\hline 30 & $1.000 \mathrm{E}+00$ & $9.983 \mathrm{E}-01$ & $2.200 \mathrm{E}-03$ & $0.000 E+00$ & $0.000 E+00$ & $0.000 E+00$ & $0.000 E+00$ \\
\hline 35 & $1.000 \mathrm{E}+00$ & $9.980 \mathrm{E}-01$ & 1.900E-03 & $0.000 E+00$ & $0.000 E+00$ & $0.000 E+00$ & $0.000 E+00$ \\
\hline 40 & $1.000 \mathrm{E}+00$ & 9.977E-01 & 1.600E-03 & $0.000 E+00$ & $0.000 E+00$ & $0.000 E+00$ & $0.000 E+00$ \\
\hline 45 & $1.000 E+00$ & $9.974 \mathrm{E}-01$ & 1.400E-03 & $0.000 E+00$ & $0.000 E+00$ & $0.000 E+00$ & $0.000 E+00$ \\
\hline 50 & $1.000 E+00$ & 9.971E-01 & 1.300E-03 & $0.000 E+00$ & $0.000 E+00$ & $0.000 E+00$ & $0.000 E+00$ \\
\hline 55 & $1.000 E+00$ & $9.968 \mathrm{E}-01$ & 1.200E-03 & $0.000 E+00$ & $0.000 E+00$ & $0.000 E+00$ & $0.000 E+00$ \\
\hline 60 & $1.000 E+00$ & $9.965 \mathrm{E}-01$ & 1.100E-03 & $0.000 E+00$ & $0.000 E+00$ & $0.000 E+00$ & $0.000 E+00$ \\
\hline 65 & $1.000 \mathrm{E}+00$ & $9.962 \mathrm{E}-01$ & $1.000 \mathrm{E}-03$ & $0.000 E+00$ & $0.000 E+00$ & $0.000 E+00$ & $0.000 E+00$ \\
\hline 70 & $1.000 \mathrm{E}+00$ & 9.959E-01 & $9.000 \mathrm{E}-04$ & $0.000 E+00$ & $0.000 E+00$ & $0.000 E+00$ & $0.000 E+00$ \\
\hline 75 & $1.000 E+00$ & $9.957 \mathrm{E}-01$ & $9.000 \mathrm{E}-04$ & $0.000 E+00$ & $0.000 E+00$ & $0.000 E+00$ & $0.000 E+00$ \\
\hline 80 & $1.000 E+00$ & $9.954 \mathrm{E}-01$ & 8.000E-04 & $0.000 E+00$ & $0.000 E+00$ & $0.000 E+00$ & $0.000 E+00$ \\
\hline 85 & $1.000 E+00$ & 9.951E-01 & $8.000 \mathrm{E}-04$ & $0.000 E+00$ & $0.000 E+00$ & $0.000 E+00$ & $0.000 E+00$ \\
\hline 90 & $1.000 \mathrm{E}+00$ & $9.948 \mathrm{E}-01$ & 7.000E-04 & $0.000 E+00$ & $0.000 E+00$ & $0.000 E+00$ & $0.000 E+00$ \\
\hline 95 & $1.000 E+00$ & $9.945 \mathrm{E}-01$ & 7.000E-04 & $0.000 E+00$ & $0.000 E+00$ & $0.000 E+00$ & $0.000 E+00$ \\
\hline 100 & $1.000 E+00$ & $9.942 \mathrm{E}-01$ & $6.000 \mathrm{E}-04$ & $0.000 E+00$ & $0.000 E+00$ & $0.000 E+00$ & $0.000 E+00$ \\
\hline 200 & $1.000 \mathrm{E}+00$ & $9.885 \mathrm{E}-01$ & 3.000E-04 & $0.000 E+00$ & $0.000 E+00$ & $0.000 E+00$ & $0.000 E+00$ \\
\hline 300 & $1.000 E+00$ & $9.829 \mathrm{E}-01$ & $2.000 \mathrm{E}-04$ & $0.000 E+00$ & $0.000 E+00$ & $0.000 E+00$ & $0.000 E+00$ \\
\hline 400 & $1.000 E+00$ & $9.773 \mathrm{E}-01$ & $2.000 \mathrm{E}-04$ & $0.000 E+00$ & $0.000 E+00$ & $0.000 E+00$ & $0.000 \mathrm{E}+00$ \\
\hline 500 & $1.000 E+00$ & $9.718 \mathrm{E}-01$ & $1.000 \mathrm{E}-04$ & $0.000 E+00$ & $0.000 E+00$ & $0.000 E+00$ & $0.000 \mathrm{E}+00$ \\
\hline 600 & $1.000 E+00$ & $9.663 \mathrm{E}-01$ & $1.000 \mathrm{E}-04$ & $0.000 E+00$ & $0.000 E+00$ & $0.000 E+00$ & $0.000 E+00$ \\
\hline 700 & $1.000 E+00$ & $9.609 \mathrm{E}-01$ & $1.000 \mathrm{E}-04$ & $0.000 \mathrm{E}+00$ & $0.000 \mathrm{E}+00$ & $0.000 E+00$ & $0.000 \mathrm{E}+00$ \\
\hline 800 & $1.000 \mathrm{E}+00$ & $9.556 \mathrm{E}-01$ & $1.000 \mathrm{E}-04$ & $0.000 E+00$ & $0.000 E+00$ & $0.000 E+00$ & $0.000 E+00$ \\
\hline 900 & $1.000 E+00$ & $9.503 E-01$ & $1.000 \mathrm{E}-04$ & $0.000 E+00$ & $0.000 E+00$ & $0.000 E+00$ & $0.000 E+00$ \\
\hline
\end{tabular}

a 0.18 is the residential default $\mathrm{ACH}$, and 0.6 is the commercial/industrial default $\mathrm{ACH}$. 
Table B-3: Radon Inhalation Fractional Equilibrium Factors Based on Air Changes Per Hour

\begin{tabular}{|c|c|c|c|c|c|c|c|c|c|}
\hline $\mathbf{A C H}^{\mathbf{a}}$ & Rn-222 & Po-218 & $\mathrm{Pb}-214$ & At-218 & Bi-214 & Rn-218 & Po-214 & Tl-210 & FEQ \\
\hline 0 & $1.000 \mathrm{E}+00$ & $1.000 \mathrm{E}+00$ & $1.000 \mathrm{E}+00$ & $2.000 \mathrm{E}-04$ & $1.000 \mathrm{E}+00$ & 2.000E-07 & $1.000 \mathrm{E}+00$ & $2.100 \mathrm{E}-04$ & $1.000 \mathrm{E}+00$ \\
\hline 0.1 & $1.000 E+00$ & $9.926 \mathrm{E}-01$ & $9.325 \mathrm{E}-01$ & $1.985 \mathrm{E}-04$ & $8.901 \mathrm{E}-01$ & $1.985 \mathrm{E}-07$ & $8.901 \mathrm{E}-01$ & $1.863 \mathrm{E}-04$ & $9.350 \mathrm{E}-01$ \\
\hline 0.18 & $1.000 \mathrm{E}+00$ & $9.868 \mathrm{E}-01$ & $8.842 \mathrm{E}-01$ & $1.973 \mathrm{E}-04$ & 8.143E-01 & $1.973 \mathrm{E}-07$ & 8.143E-01 & 1.700E-04 & 8.899E-01 \\
\hline 0.2 & $1.000 \mathrm{E}+00$ & $9.853 \mathrm{E}-01$ & $8.728 \mathrm{E}-01$ & $1.970 \mathrm{E}-04$ & 7.968E-01 & $1.970 \mathrm{E}-07$ & 7.968E-01 & $1.663 \mathrm{E}-04$ & 8.794E-01 \\
\hline 0.3 & $1.000 E+00$ & $9.781 \mathrm{E}-01$ & 8.197E-01 & $1.956 \mathrm{E}-04$ & 7.169E-01 & $1.956 \mathrm{E}-07$ & 7.169E-01 & $1.492 \mathrm{E}-04$ & $8.314 \mathrm{E}-01$ \\
\hline 0.4 & $1.000 \mathrm{E}+00$ & $9.710 \mathrm{E}-01$ & $7.720 \mathrm{E}-01$ & $1.942 \mathrm{E}-04$ & $6.482 \mathrm{E}-01$ & $1.942 \mathrm{E}-07$ & $6.482 \mathrm{E}-01$ & $1.344 \mathrm{E}-04$ & 7.897E-01 \\
\hline 0.5 & $1.000 \mathrm{E}+00$ & $9.641 \mathrm{E}-01$ & $7.291 \mathrm{E}-01$ & $1.928 \mathrm{E}-04$ & $5.885 \mathrm{E}-01$ & $1.928 \mathrm{E}-07$ & $5.885 \mathrm{E}-01$ & $1.217 \mathrm{E}-04$ & 7.532E-01 \\
\hline 0.6 & $1.000 \mathrm{E}+00$ & $9.572 \mathrm{E}-01$ & $6.903 \mathrm{E}-01$ & $1.914 \mathrm{E}-04$ & $5.365 \mathrm{E}-01$ & $1.914 \mathrm{E}-07$ & $5.365 \mathrm{E}-01$ & 1.106E-04 & 7.209E-01 \\
\hline 0.7 & $1.000 E+00$ & $9.504 \mathrm{E}-01$ & $6.550 \mathrm{E}-01$ & $1.900 \mathrm{E}-04$ & $4.908 \mathrm{E}-01$ & $1.900 \mathrm{E}-07$ & $4.908 \mathrm{E}-01$ & 1.009E-04 & $6.923 \mathrm{E}-01$ \\
\hline 0.8 & $1.000 \mathrm{E}+00$ & $9.437 \mathrm{E}-01$ & $6.227 \mathrm{E}-01$ & $1.887 \mathrm{E}-04$ & $4.505 \mathrm{E}-01$ & $1.887 \mathrm{E}-07$ & 4.505E-01 & $9.229 E-05$ & $6.667 \mathrm{E}-01$ \\
\hline 0.9 & $1.000 \mathrm{E}+00$ & $9.371 \mathrm{E}-01$ & 5.931E-01 & $1.873 \mathrm{E}-04$ & 4.147E-01 & $1.873 \mathrm{E}-07$ & 4.147E-01 & $8.471 \mathrm{E}-05$ & $6.437 \mathrm{E}-01$ \\
\hline 1 & $1.000 E+00$ & $9.306 \mathrm{E}-01$ & $5.659 \mathrm{E}-01$ & $1.860 \mathrm{E}-04$ & $3.829 E-01$ & $1.860 \mathrm{E}-07$ & 3.829E-01 & 7.797E-05 & $6.230 \mathrm{E}-01$ \\
\hline 1.1 & $1.000 \mathrm{E}+00$ & $9.242 \mathrm{E}-01$ & $5.408 \mathrm{E}-01$ & $1.847 \mathrm{E}-04$ & $3.545 \mathrm{E}-01$ & $1.847 \mathrm{E}-07$ & $3.545 \mathrm{E}-01$ & 7.196E-05 & $6.042 \mathrm{E}-01$ \\
\hline 1.2 & $1.000 E+00$ & $9.179 E-01$ & $5.176 \mathrm{E}-01$ & $1.834 \mathrm{E}-04$ & $3.289 \mathrm{E}-01$ & $1.834 \mathrm{E}-07$ & $3.289 \mathrm{E}-01$ & $6.658 \mathrm{E}-05$ & $5.871 \mathrm{E}-01$ \\
\hline 1.3 & $1.000 E+00$ & 9.117E-01 & $4.961 \mathrm{E}-01$ & $1.822 \mathrm{E}-04$ & $3.059 \mathrm{E}-01$ & $1.822 \mathrm{E}-07$ & 3.059E-01 & $6.174 \mathrm{E}-05$ & $5.715 \mathrm{E}-01$ \\
\hline 1.4 & $1.000 E+00$ & $9.055 \mathrm{E}-01$ & $4.760 \mathrm{E}-01$ & $1.809 \mathrm{E}-04$ & $2.852 \mathrm{E}-01$ & $1.809 \mathrm{E}-07$ & $2.852 \mathrm{E}-01$ & $5.738 \mathrm{E}-05$ & $5.571 \mathrm{E}-01$ \\
\hline 1.5 & $1.000 \mathrm{E}+00$ & 8.994E-01 & $4.574 \mathrm{E}-01$ & $1.797 \mathrm{E}-04$ & $2.664 \mathrm{E}-01$ & 1.797E-07 & $2.664 \mathrm{E}-01$ & $5.343 \mathrm{E}-05$ & $5.439 \mathrm{E}-01$ \\
\hline 1.6 & $1.000 \mathrm{E}+00$ & 8.934E-01 & 4.399E-01 & $1.785 \mathrm{E}-04$ & 2.492E-01 & $1.785 \mathrm{E}-07$ & $2.492 \mathrm{E}-01$ & 4.985E-05 & 5.316E-01 \\
\hline 1.7 & $1.000 \mathrm{E}+00$ & $8.875 \mathrm{E}-01$ & 4.235E-01 & $1.773 \mathrm{E}-04$ & $2.337 \mathrm{E}-01$ & $1.773 \mathrm{E}-07$ & 2.337E-01 & $4.659 \mathrm{E}-05$ & $5.203 E-01$ \\
\hline 1.8 & $1.000 \mathrm{E}+00$ & $8.817 \mathrm{E}-01$ & $4.082 \mathrm{E}-01$ & $1.761 \mathrm{E}-04$ & 2.194E-01 & $1.761 \mathrm{E}-07$ & $2.194 \mathrm{E}-01$ & $4.362 \mathrm{E}-05$ & 5.097E-01 \\
\hline 1.9 & $1.000 \mathrm{E}+00$ & $8.759 E-01$ & $3.938 \mathrm{E}-01$ & $1.750 \mathrm{E}-04$ & $2.064 \mathrm{E}-01$ & $1.750 \mathrm{E}-07$ & $2.064 \mathrm{E}-01$ & 4.091E-05 & 4.999E-01 \\
\hline 2 & $1.000 \mathrm{E}+00$ & 8.703E-01 & $3.802 \mathrm{E}-01$ & $1.738 \mathrm{E}-04$ & $1.944 \mathrm{E}-01$ & $1.738 \mathrm{E}-07$ & $1.944 \mathrm{E}-01$ & $3.842 \mathrm{E}-05$ & 4.907E-01 \\
\hline 2.1 & $1.000 \mathrm{E}+00$ & 8.647E-01 & $3.674 \mathrm{E}-01$ & $1.727 \mathrm{E}-04$ & $1.834 \mathrm{E}-01$ & $1.727 \mathrm{E}-07$ & $1.834 \mathrm{E}-01$ & $3.613 \mathrm{E}-05$ & $4.820 \mathrm{E}-01$ \\
\hline 2.2 & $1.000 E+00$ & $8.591 \mathrm{E}-01$ & $3.553 \mathrm{E}-01$ & $1.716 \mathrm{E}-04$ & $1.732 \mathrm{E}-01$ & $1.716 \mathrm{E}-07$ & $1.732 \mathrm{E}-01$ & $3.403 \mathrm{E}-05$ & 4.739E-01 \\
\hline 2.3 & $1.000 \mathrm{E}+00$ & $8.537 \mathrm{E}-01$ & $3.439 \mathrm{E}-01$ & 1.705E-04 & $1.638 \mathrm{E}-01$ & $1.705 \mathrm{E}-07$ & $1.638 \mathrm{E}-01$ & $3.209 E-05$ & 4.662E-01 \\
\hline 2.4 & $1.000 \mathrm{E}+00$ & $8.483 \mathrm{E}-01$ & 3.331E-01 & $1.694 \mathrm{E}-04$ & $1.551 \mathrm{E}-01$ & $1.694 \mathrm{E}-07$ & $1.551 \mathrm{E}-01$ & $3.030 \mathrm{E}-05$ & $4.590 \mathrm{E}-01$ \\
\hline 2.5 & $1.000 \mathrm{E}+00$ & $8.429 \mathrm{E}-01$ & $3.228 \mathrm{E}-01$ & 1.683E-04 & $1.471 \mathrm{E}-01$ & $1.683 \mathrm{E}-07$ & $1.471 \mathrm{E}-01$ & $2.865 \mathrm{E}-05$ & $4.521 \mathrm{E}-01$ \\
\hline 2.6 & $1.000 \mathrm{E}+00$ & 8.377E-01 & $3.131 \mathrm{E}-01$ & 1.673E-04 & 1.396E-01 & 1.673E-07 & 1.396E-01 & $2.711 \mathrm{E}-05$ & 4.456E-01 \\
\hline 2.7 & $1.000 \mathrm{E}+00$ & $8.325 \mathrm{E}-01$ & $3.038 \mathrm{E}-01$ & $1.662 \mathrm{E}-04$ & $1.326 \mathrm{E}-01$ & $1.662 \mathrm{E}-07$ & $1.326 \mathrm{E}-01$ & $2.569 \mathrm{E}-05$ & 4.394E-01 \\
\hline 2.8 & $1.000 E+00$ & $8.273 \mathrm{E}-01$ & $2.950 \mathrm{E}-01$ & $1.652 \mathrm{E}-04$ & $1.262 \mathrm{E}-01$ & $1.652 \mathrm{E}-07$ & $1.262 \mathrm{E}-01$ & 2.436E-05 & 4.335E-01 \\
\hline 2.9 & $1.000 \mathrm{E}+00$ & $8.223 \mathrm{E}-01$ & $2.866 \mathrm{E}-01$ & $1.642 \mathrm{E}-04$ & $1.201 \mathrm{E}-01$ & $1.642 \mathrm{E}-07$ & $1.201 \mathrm{E}-01$ & $2.313 \mathrm{E}-05$ & 4.279E-01 \\
\hline 3 & $1.000 \mathrm{E}+00$ & $8.172 \mathrm{E}-01$ & $2.786 \mathrm{E}-01$ & $1.632 \mathrm{E}-04$ & $1.145 \mathrm{E}-01$ & $1.631 \mathrm{E}-07$ & $1.145 \mathrm{E}-01$ & $2.198 \mathrm{E}-05$ & 4.225E-01 \\
\hline 3.1 & $1.000 \mathrm{E}+00$ & $8.123 \mathrm{E}-01$ & $2.710 \mathrm{E}-01$ & $1.622 \mathrm{E}-04$ & $1.092 \mathrm{E}-01$ & $1.622 \mathrm{E}-07$ & $1.092 \mathrm{E}-01$ & 2.090E-05 & 4.174E-01 \\
\hline 3.2 & $1.000 E+00$ & $8.074 \mathrm{E}-01$ & 2.637E-01 & $1.612 \mathrm{E}-04$ & $1.042 \mathrm{E}-01$ & $1.612 \mathrm{E}-07$ & $1.042 \mathrm{E}-01$ & $1.990 \mathrm{E}-05$ & 4.125E-01 \\
\hline 3.3 & $1.000 E+00$ & $8.026 \mathrm{E}-01$ & 2.567E-01 & $1.602 \mathrm{E}-04$ & $9.960 \mathrm{E}-02$ & $1.602 \mathrm{E}-07$ & $9.960 \mathrm{E}-02$ & 1.896E-05 & 4.078E-01 \\
\hline 3.4 & $1.000 E+00$ & 7.978E-01 & $2.500 \mathrm{E}-01$ & $1.592 \mathrm{E}-04$ & $9.524 \mathrm{E}-02$ & $1.592 \mathrm{E}-07$ & $9.524 \mathrm{E}-02$ & $1.808 \mathrm{E}-05$ & 4.032E-01 \\
\hline 3.5 & $1.000 \mathrm{E}+00$ & 7.931E-01 & $2.436 \mathrm{E}-01$ & $1.583 \mathrm{E}-04$ & $9.114 \mathrm{E}-02$ & $1.583 \mathrm{E}-07$ & $9.114 \mathrm{E}-02$ & $1.725 \mathrm{E}-05$ & $3.988 \mathrm{E}-01$ \\
\hline 3.6 & $1.000 \mathrm{E}+00$ & $7.884 \mathrm{E}-01$ & $2.375 \mathrm{E}-01$ & $1.573 \mathrm{E}-04$ & $8.729 \mathrm{E}-02$ & $1.573 \mathrm{E}-07$ & $8.729 E-02$ & $1.648 \mathrm{E}-05$ & $3.946 \mathrm{E}-01$ \\
\hline 3.7 & $1.000 \mathrm{E}+00$ & $7.838 \mathrm{E}-01$ & $2.316 \mathrm{E}-01$ & $1.564 \mathrm{E}-04$ & $8.366 \mathrm{E}-02$ & $1.564 \mathrm{E}-07$ & $8.366 \mathrm{E}-02$ & $1.575 \mathrm{E}-05$ & $3.906 \mathrm{E}-01$ \\
\hline
\end{tabular}




\begin{tabular}{|c|c|c|c|c|c|c|c|c|c|}
\hline $\mathbf{A C H}^{\mathrm{a}}$ & Rn-222 & Po-218 & Pb-214 & At-218 & Bi-214 & Rn-218 & Po-214 & Tl-210 & FEQ \\
\hline 3.8 & $1.000 \mathrm{E}+00$ & 7.793E-01 & $2.260 \mathrm{E}-01$ & $1.555 \mathrm{E}-04$ & $8.023 \mathrm{E}-02$ & $1.555 \mathrm{E}-07$ & $8.023 E-02$ & $1.506 \mathrm{E}-05$ & 3.867E-01 \\
\hline 3.9 & $1.000 \mathrm{E}+00$ & $7.748 \mathrm{E}-01$ & $2.205 \mathrm{E}-01$ & 1.546E-04 & 7.700E-02 & $1.546 \mathrm{E}-07$ & $7.700 \mathrm{E}-02$ & $1.441 \mathrm{E}-05$ & $3.829 \mathrm{E}-01$ \\
\hline 4 & $1.000 E+00$ & 7.703E-01 & $2.153 \mathrm{E}-01$ & $1.537 \mathrm{E}-04$ & 7.394E-02 & 1.537E-07 & 7.394E-02 & $1.380 \mathrm{E}-05$ & 3.792E-01 \\
\hline 5 & $1.000 \mathrm{E}+00$ & $7.285 \mathrm{E}-01$ & $1.725 \mathrm{E}-01$ & $1.453 \mathrm{E}-04$ & $5.090 \mathrm{E}-02$ & $1.453 \mathrm{E}-07$ & $5.090 \mathrm{E}-02$ & $9.245 \mathrm{E}-06$ & $3.479 \mathrm{E}-01$ \\
\hline 6 & $1.000 E+00$ & $6.910 \mathrm{E}-01$ & $1.420 \mathrm{E}-01$ & $1.377 \mathrm{E}-04$ & $3.672 \mathrm{E}-02$ & 1.377E-07 & $3.672 \mathrm{E}-02$ & $6.493 \mathrm{E}-06$ & $3.235 \mathrm{E}-01$ \\
\hline 7 & $1.000 \mathrm{E}+00$ & $6.571 \mathrm{E}-01$ & $1.192 \mathrm{E}-01$ & $1.309 \mathrm{E}-04$ & $2.745 \mathrm{E}-02$ & 1.309E-07 & $2.745 \mathrm{E}-02$ & 4.729E-06 & $3.035 E-01$ \\
\hline 8 & $1.000 \mathrm{E}+00$ & $6.264 \mathrm{E}-01$ & $1.018 \mathrm{E}-01$ & $1.247 \mathrm{E}-04$ & $2.111 \mathrm{E}-02$ & 1.247E-07 & $2.111 \mathrm{E}-02$ & $3.546 \mathrm{E}-06$ & $2.865 \mathrm{E}-01$ \\
\hline 9 & $1.000 \mathrm{E}+00$ & $5.985 \mathrm{E}-01$ & $8.802 \mathrm{E}-02$ & $1.191 \mathrm{E}-04$ & $1.661 \mathrm{E}-02$ & 1.190E-07 & $1.661 \mathrm{E}-02$ & $2.722 \mathrm{E}-06$ & 2.717E-01 \\
\hline 10 & $1.000 \mathrm{E}+00$ & $5.729 \mathrm{E}-01$ & 7.697E-02 & $1.139 \mathrm{E}-04$ & $1.332 \mathrm{E}-02$ & $1.139 \mathrm{E}-07$ & $1.332 \mathrm{E}-02$ & $2.132 \mathrm{E}-06$ & $2.586 \mathrm{E}-01$ \\
\hline 15 & $1.000 E+00$ & $4.721 \mathrm{E}-01$ & $4.426 \mathrm{E}-02$ & $9.358 \mathrm{E}-05$ & $5.424 \mathrm{E}-03$ & $9.356 \mathrm{E}-08$ & $5.424 \mathrm{E}-03$ & 7.755E-07 & $2.100 \mathrm{E}-01$ \\
\hline 20 & $1.000 E+00$ & 4.015E-01 & 2.891E-02 & 7.934E-05 & $2.742 \mathrm{E}-03$ & 7.932E-08 & $2.743 \mathrm{E}-03$ & $3.544 \mathrm{E}-07$ & $1.775 \mathrm{E}-01$ \\
\hline 25 & $1.000 \mathrm{E}+00$ & $3.492 \mathrm{E}-01$ & $2.041 \mathrm{E}-02$ & $6.881 \mathrm{E}-05$ & $1.580 \mathrm{E}-03$ & $6.879 \mathrm{E}-08$ & $1.580 \mathrm{E}-03$ & $1.862 \mathrm{E}-07$ & $1.540 \mathrm{E}-01$ \\
\hline 30 & $1.000 \mathrm{E}+00$ & $3.090 \mathrm{E}-01$ & $1.520 \mathrm{E}-02$ & $6.071 \mathrm{E}-05$ & $9.937 \mathrm{E}-04$ & $6.068 \mathrm{E}-08$ & $9.938 \mathrm{E}-04$ & $1.077 \mathrm{E}-07$ & $1.360 \mathrm{E}-01$ \\
\hline 35 & $1.000 E+00$ & $2.771 \mathrm{E}-01$ & $1.176 \mathrm{E}-02$ & $5.428 \mathrm{E}-05$ & $6.659 \mathrm{E}-04$ & $5.425 \mathrm{E}-08$ & $6.660 \mathrm{E}-04$ & $6.678 \mathrm{E}-08$ & $1.218 \mathrm{E}-01$ \\
\hline 40 & $1.000 E+00$ & $2.512 \mathrm{E}-01$ & $9.380 \mathrm{E}-03$ & 4.905E-05 & $4.682 \mathrm{E}-04$ & $4.902 \mathrm{E}-08$ & $4.682 \mathrm{E}-04$ & 4.369E-08 & $1.104 \mathrm{E}-01$ \\
\hline 45 & $1.000 \mathrm{E}+00$ & 2.297E-01 & 7.656E-03 & 4.472E-05 & $3.418 \mathrm{E}-04$ & 4.469E-08 & $3.418 \mathrm{E}-04$ & $2.982 \mathrm{E}-08$ & $1.009 \mathrm{E}-01$ \\
\hline 50 & $1.000 \mathrm{E}+00$ & $2.116 \mathrm{E}-01$ & $6.368 \mathrm{E}-03$ & 4.108E-05 & $2.571 \mathrm{E}-04$ & 4.105E-08 & $2.572 \mathrm{E}-04$ & $2.107 \mathrm{E}-08$ & $9.288 \mathrm{E}-02$ \\
\hline 55 & $1.000 \mathrm{E}+00$ & $1.961 \mathrm{E}-01$ & $5.381 \mathrm{E}-03$ & 3.796E-05 & $1.984 \mathrm{E}-04$ & 3.793E-08 & $1.984 \mathrm{E}-04$ & $1.532 \mathrm{E}-08$ & 8.607E-02 \\
\hline 60 & $1.000 \mathrm{E}+00$ & $1.827 \mathrm{E}-01$ & 4.607E-03 & $3.528 \mathrm{E}-05$ & $1.563 \mathrm{E}-04$ & $3.525 \mathrm{E}-08$ & $1.563 \mathrm{E}-04$ & $1.141 \mathrm{E}-08$ & 8.019E-02 \\
\hline 65 & $1.000 \mathrm{E}+00$ & $1.711 \mathrm{E}-01$ & $3.989 \mathrm{E}-03$ & $3.293 \mathrm{E}-05$ & $1.253 \mathrm{E}-04$ & $3.290 \mathrm{E}-08$ & $1.253 \mathrm{E}-04$ & 8.679E-09 & 7.507E-02 \\
\hline 70 & $1.000 \mathrm{E}+00$ & $1.608 \mathrm{E}-01$ & $3.488 \mathrm{E}-03$ & $3.087 \mathrm{E}-05$ & $1.020 \mathrm{E}-04$ & $3.084 \mathrm{E}-08$ & $1.020 \mathrm{E}-04$ & $6.720 \mathrm{E}-09$ & $7.056 \mathrm{E}-02$ \\
\hline 75 & $1.000 \mathrm{E}+00$ & $1.517 \mathrm{E}-01$ & $3.076 \mathrm{E}-03$ & 2.904E-05 & 8.417E-05 & $2.901 \mathrm{E}-08$ & $8.420 \mathrm{E}-05$ & $5.285 \mathrm{E}-09$ & $6.656 \mathrm{E}-02$ \\
\hline 80 & $1.000 \mathrm{E}+00$ & $1.436 \mathrm{E}-01$ & $2.733 \mathrm{E}-03$ & 2.740E-05 & 7.027E-05 & 2.737E-08 & $7.030 \mathrm{E}-05$ & 4.215E-09 & $6.299 \mathrm{E}-02$ \\
\hline 85 & $1.000 \mathrm{E}+00$ & $1.363 \mathrm{E}-01$ & $2.444 \mathrm{E}-03$ & $2.594 \mathrm{E}-05$ & 5.927E-05 & $2.591 \mathrm{E}-08$ & $5.930 \mathrm{E}-05$ & $3.404 \mathrm{E}-09$ & $5.979 \mathrm{E}-02$ \\
\hline 90 & $1.000 E+00$ & $1.297 \mathrm{E}-01$ & 2.199E-03 & $2.461 \mathrm{E}-05$ & 5.046E-05 & $2.458 \mathrm{E}-08$ & $5.048 \mathrm{E}-05$ & 2.779E-09 & $5.689 \mathrm{E}-02$ \\
\hline 95 & $1.000 \mathrm{E}+00$ & $1.237 \mathrm{E}-01$ & $1.989 \mathrm{E}-03$ & $2.341 \mathrm{E}-05$ & 4.331E-05 & $2.338 \mathrm{E}-08$ & $4.334 \mathrm{E}-05$ & $2.291 \mathrm{E}-09$ & 5.427E-02 \\
\hline 100 & $1.000 \mathrm{E}+00$ & $1.183 \mathrm{E}-01$ & $1.808 \mathrm{E}-03$ & $2.232 \mathrm{E}-05$ & 3.746E-05 & $2.228 \mathrm{E}-08$ & $3.748 \mathrm{E}-05$ & 1.907E-09 & 5.187E-02 \\
\hline 200 & $1.000 \mathrm{E}+00$ & $6.286 \mathrm{E}-02$ & $4.840 \mathrm{E}-04$ & $1.122 \mathrm{E}-05$ & $5.121 \mathrm{E}-06$ & 1.119E-08 & $5.132 \mathrm{E}-06$ & $1.483 \mathrm{E}-10$ & $2.756 \mathrm{E}-02$ \\
\hline 300 & $1.000 E+00$ & 4.280E-02 & $2.203 E-04$ & 7.253E-06 & $1.574 \mathrm{E}-06$ & 7.223E-09 & $1.581 \mathrm{E}-06$ & $3.185 \mathrm{E}-11$ & $1.876 \mathrm{E}-02$ \\
\hline 400 & $1.000 \mathrm{E}+00$ & $3.245 \mathrm{E}-02$ & $1.254 \mathrm{E}-04$ & $5.232 \mathrm{E}-06$ & $6.790 \mathrm{E}-07$ & 5.203E-09 & $6.842 \mathrm{E}-07$ & $1.056 \mathrm{E}-11$ & $1.423 \mathrm{E}-02$ \\
\hline 500 & $1.000 \mathrm{E}+00$ & $2.613 \mathrm{E}-02$ & $8.085 \mathrm{E}-05$ & $4.018 \mathrm{E}-06$ & $3.532 \mathrm{E}-07$ & 3.990E-09 & $3.572 \mathrm{E}-07$ & $4.461 \mathrm{E}-12$ & $1.145 \mathrm{E}-02$ \\
\hline 600 & $1.000 E+00$ & $2.187 \mathrm{E}-02$ & $5.642 \mathrm{E}-05$ & $3.215 \mathrm{E}-06$ & $2.070 \mathrm{E}-07$ & $3.188 \mathrm{E}-09$ & $2.102 \mathrm{E}-07$ & $2.200 \mathrm{E}-12$ & $9.587 \mathrm{E}-03$ \\
\hline 700 & $1.000 \mathrm{E}+00$ & $1.880 \mathrm{E}-02$ & 4.160E-05 & $2.647 \mathrm{E}-06$ & 1.317E-07 & $2.621 \mathrm{E}-09$ & $1.343 \mathrm{E}-07$ & $1.209 \mathrm{E}-12$ & 8.243E-03 \\
\hline 800 & $1.000 \mathrm{E}+00$ & 1.649E-02 & 3.193E-05 & $2.227 \mathrm{E}-06$ & $8.900 \mathrm{E}-08$ & 2.203E-09 & $9.119 \mathrm{E}-08$ & $7.186 \mathrm{E}-13$ & $7.229 \mathrm{E}-03$ \\
\hline 900 & $1.000 \mathrm{E}+00$ & $1.469 \mathrm{E}-02$ & $2.528 \mathrm{E}-05$ & 1.906E-06 & $6.298 \mathrm{E}-08$ & $1.882 \mathrm{E}-09$ & $6.486 \mathrm{E}-08$ & $4.540 \mathrm{E}-13$ & $6.438 \mathrm{E}-03$ \\
\hline
\end{tabular}

${ }^{\mathrm{a}} 0.18$ is the residential default $\mathrm{ACH}$, and 0.6 is the commercial/industrial default $\mathrm{ACH}$. 
APPENDIX C. ACTINON, THORON, AND RADON DECAY SERIES DATA 

Table C-1: Actinon Decay Series

\begin{tabular}{|c|c|c|c|c|c|c|c|}
\hline Nuclide & Half-life & Mode & Mass & $\begin{array}{c}\text { Branching } \\
\text { Fraction }\end{array}$ & Daughter & $\begin{array}{l}\text { Branching } \\
\text { Fraction }\end{array}$ & Daughter \\
\hline Rn-219 & $3.96 \mathrm{~s}$ & A & 219.00948 & $1.00 \mathrm{E}+00$ & Po-215 & & \\
\hline Po-215 & $1.781 \mathrm{E}-3 \mathrm{~s}$ & $A$ & 214.999419 & $1.00 E+00$ & $\mathrm{~Pb}-211$ & & \\
\hline $\mathrm{Pb}-211$ & $36.1 \mathrm{~m}$ & B- & 210.988736 & $1.00 \mathrm{E}+00$ & $\mathrm{Bi}-211$ & & \\
\hline Bi-211 & $2.14 \mathrm{~m}$ & $B-, A$ & 210.987269 & 9.97E-01 & TI-207 & $2.76 \mathrm{E}-03$ & Po-211 \\
\hline TI-207 & $4.77 \mathrm{~m}$ & B- & 206.977419 & $1.00 \mathrm{E}+00$ & $\begin{array}{c}\text { Stable Pb- } \\
207\end{array}$ & & \\
\hline Po-211 & $0.516 \mathrm{~s}$ & $A$ & 210.986653 & $1.00 \mathrm{E}+00$ & $\begin{array}{c}\text { Stable Pb- } \\
207\end{array}$ & & \\
\hline
\end{tabular}

Table C-2: Actinon Alpha Energies Released Per Decay

\begin{tabular}{|c|c|c|c|c|c|}
\hline \multicolumn{7}{|c|}{ Alpha Energy (MeV) } \\
\hline Rn-219 & Po-215 & Pb-211 & Bi-211 & Tl-207 & Po-211 \\
\hline 6.8801 & 7.5261 & 0 & 6.6756 & 0 & 7.586 \\
\hline
\end{tabular}

Table C-3: Thoron Decay Series

\begin{tabular}{|c|c|c|c|c|c|c|c|}
\hline Nuclide & Half-life & Mode & Mass & $\begin{array}{c}\text { Branching } \\
\text { Fraction }\end{array}$ & Daughter & $\begin{array}{c}\text { Branching } \\
\text { Fraction }\end{array}$ & Daughter \\
\hline $\mathrm{Rn}-220$ & $55.6 \mathrm{~s}$ & $A$ & 220.011393 & $1.00 \mathrm{E}+00$ & Po-216 & & \\
\hline Po-216 & $0.145 \mathrm{~s}$ & $A$ & 216.001915 & $1.00 \mathrm{E}+00$ & $\mathrm{~Pb}-212$ & & \\
\hline $\mathrm{Pb}-212$ & $10.64 \mathrm{~h}$ & B- & 211.991897 & $1.00 \mathrm{E}+00$ & $\mathrm{Bi}-212$ & & \\
\hline Bi-212 & $60.55 \mathrm{~m}$ & $B-, A$ & 211.991285 & $6.41 \mathrm{E}-01$ & Po-212 & 3.59E-01 & TI-208 \\
\hline Po-212 & $2.99 \mathrm{E}-7 \mathrm{~s}$ & $A$ & 211.988867 & $1.00 \mathrm{E}+00$ & $\begin{array}{c}\text { Stable Pb- } \\
208\end{array}$ & & \\
\hline TI-208 & $3.053 \mathrm{~m}$ & B- & 207.982018 & $1.00 \mathrm{E}+00$ & $\begin{array}{c}\text { Stable Pb- } \\
208\end{array}$ & & \\
\hline
\end{tabular}

Table C-4: Thoron Alpha Energies Released Per Decay

\begin{tabular}{|l|l|l|l|l|l|}
\hline \multicolumn{7}{|c|}{ Alpha Energy (MeV) } \\
\hline Rn-220 & Po-216 & Pb-212 & Bi-212 & Po-212 & Tl-208 \\
\hline 6.404 & 6.9063 & 0 & 2.2163 & 8.9541 & 0 \\
\hline
\end{tabular}


Table C-5: Radon Decay Series

\begin{tabular}{|c|c|c|c|c|c|c|c|}
\hline Nuclide & Half-life & Mode & Mass & $\begin{array}{l}\text { Branching } \\
\text { Fraction }\end{array}$ & Daughter & $\begin{array}{l}\text { Branching } \\
\text { Fraction }\end{array}$ & Daughter \\
\hline Rn-222 & $3.8235 d$ & $\mathrm{~A}$ & 222.017577 & $1.00 \mathrm{E}+00$ & Po-218 & & \\
\hline Po-218 & $3.10 \mathrm{~m}$ & $B-, A$ & 218.008973 & $1.00 \mathrm{E}+00$ & $\mathrm{~Pb}-214$ & $2.00 \mathrm{E}-04$ & At-218 \\
\hline $\mathrm{Pb}-214$ & $26.8 \mathrm{~m}$ & B- & 213.999805 & $1.00 \mathrm{E}+00$ & $\mathrm{Bi}-214$ & & \\
\hline At-218 & $1.5 \mathrm{~s}$ & A, B- & 218.008694 & 9.99E-01 & $\mathrm{Bi}-214$ & $1.00 \mathrm{E}-03$ & $\mathrm{Rn}-218$ \\
\hline $\mathrm{Bi}-214$ & $19.9 \mathrm{~m}$ & $B-, A$ & 213.998711 & $1.00 \mathrm{E}+00$ & Po-214 & $2.10 \mathrm{E}-04$ & $\mathrm{Tl}-210$ \\
\hline Rn-218 & $3.5 \mathrm{E}-2 \mathrm{~s}$ & $A$ & 218.005601 & $1.00 \mathrm{E}+00$ & Po-214 & & \\
\hline Po-214 & $1.643 \mathrm{E}-4 \mathrm{~s}$ & A & 213.995201 & $1.00 \mathrm{E}+00$ & $\mathrm{~Pb}-210$ & & \\
\hline TI-210 & $1.30 \mathrm{~m}$ & B- & 209.990073 & $1.00 \mathrm{E}+00$ & $\mathrm{~Pb}-210$ & & \\
\hline $\mathrm{Pb}-210$ & $22.20 \mathrm{y}$ & $B-, A$ & 209.984188 & $1.00 \mathrm{E}+00$ & $\mathrm{Bi}-210$ & $1.90 \mathrm{E}-08$ & $\mathrm{Hg}-206$ \\
\hline $\mathrm{Bi}-210$ & $5.013 d$ & $B-, A$ & 209.98412 & $1.00 \mathrm{E}+00$ & Po-210 & $1.32 \mathrm{E}-06$ & Tl-206 \\
\hline $\mathrm{Hg}-206$ & $8.15 \mathrm{~m}$ & B- & 205.977514 & $1.00 \mathrm{E}+00$ & TI-206 & & \\
\hline Po-210 & $138.376 d$ & A & 209.982873 & $1.00 \mathrm{E}+00$ & $\begin{array}{c}\text { Stable Pb- } \\
206 \\
\end{array}$ & & \\
\hline TI-206 & $4.200 \mathrm{~m}$ & B- & 205.97611 & $1.00 \mathrm{E}+00$ & $\begin{array}{c}\text { Stable Pb- } \\
206 \\
\end{array}$ & & \\
\hline
\end{tabular}

Table C-6: Radon Alpha Energies Released Per Decay

\begin{tabular}{|c|c|c|c|c|c|c|c|c|c|c|c|c|}
\hline \multicolumn{13}{|c|}{ Alpha Energy (MeV) } \\
\hline $\begin{array}{l}\text { Rn- } \\
222\end{array}$ & $\begin{array}{l}\text { Po- } \\
218\end{array}$ & $\begin{array}{l}\mathrm{Pb}- \\
214\end{array}$ & At-218 & $\mathrm{Bi}-214$ & $\begin{array}{l}\text { Rn- } \\
218\end{array}$ & $\begin{array}{l}\text { Po- } \\
214\end{array}$ & TI-210 & $\begin{array}{l}\mathrm{Pb}- \\
210^{*}\end{array}$ & $\begin{array}{l}\mathrm{Bi}- \\
210 *\end{array}$ & $\begin{array}{l}\mathrm{Hg}- \\
206^{*}\end{array}$ & $\begin{array}{l}\text { Po- } \\
210 *\end{array}$ & $\begin{array}{l}\text { TI- } \\
\text { 206* }\end{array}$ \\
\hline 5.5898 & 6.1134 & 0 & 6.8041 & 0.0011 & 7.2618 & 7.8333 & 0 & 0 & 0 & 0 & 5.4074 & 0 \\
\hline
\end{tabular}

*These energies were not used in the $\mathrm{F}_{\mathrm{eq}}$ calculations due to $\mathrm{Pb}-210$ long half-life. 
APPENDIX D. SUPPORTING MATLAB CODE 



\section{Actinon Activity Equilibrium Factor Code}

$\%$ Actinon AEQ calculator

for AirRemovalRate $=\left[\begin{array}{llllllllllll}0 & 0.1 & 0.18 & 0.2 & 0.3 & 0.4 & 0.5 & 0.6 & 0.7 & 0.8 & 0.9 & 1\end{array}\right.$

$\begin{array}{lllllllllllllllll}1.1 & 1.2 & 1.3 & 1.4 & 1.5 & 1.6 & 1.7 & 1.8 & 1.9 & 2 & 2.1 & 2.2 & 2.3 & 2.4 & 2.5 & 2.6 & 2.7\end{array}$

$\begin{array}{lllllllllllllllllll}2.8 & 2.9 & 3 & 3.1 & 3.2 & 3.3 & 3.4 & 3.5 & 3.6 & 3.7 & 3.8 & 3.9 & 4 & 5 & 6 & 7 & 8 & 9 & 10\end{array}$

$\begin{array}{llllllllllllllllllll}15 & 20 & 25 & 30 & 35 & 40 & 45 & 50 & 55 & 60 & 65 & 70 & 75 & 80 & 85 & 90 & 95 & 100 & 200 & 300\end{array}$

$\left.\begin{array}{llllll}400 & 500 & 600 & 700 & 800 & 900\end{array}\right]$

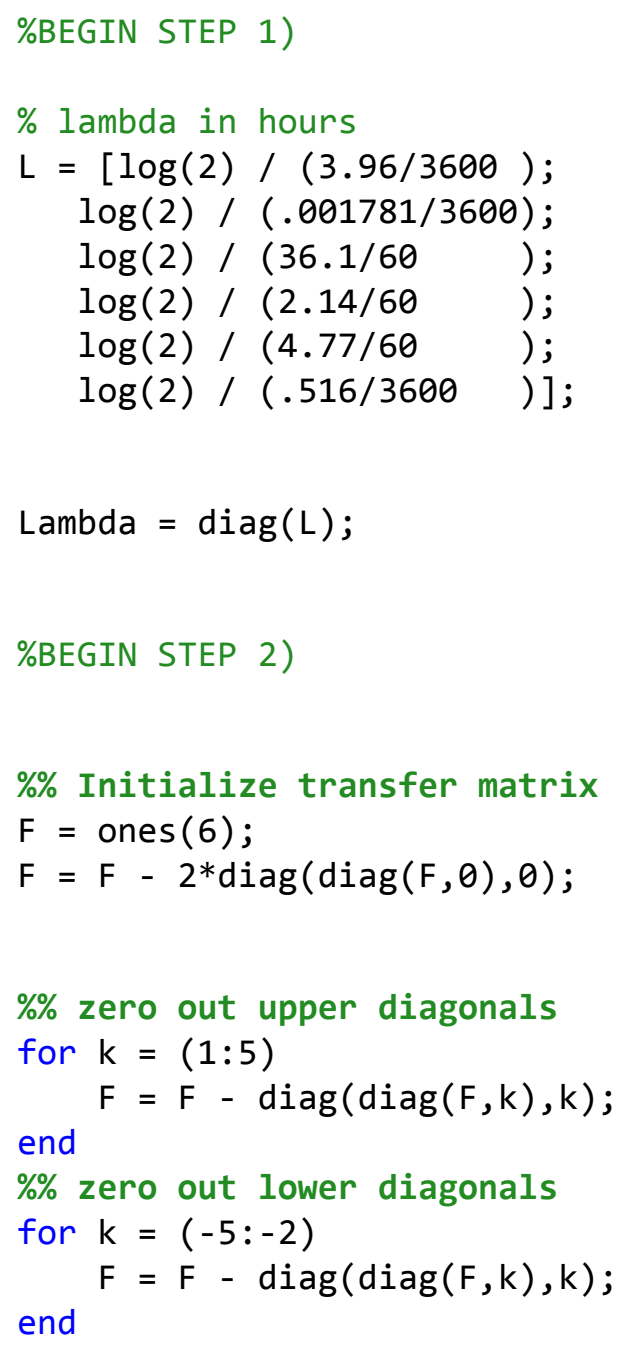




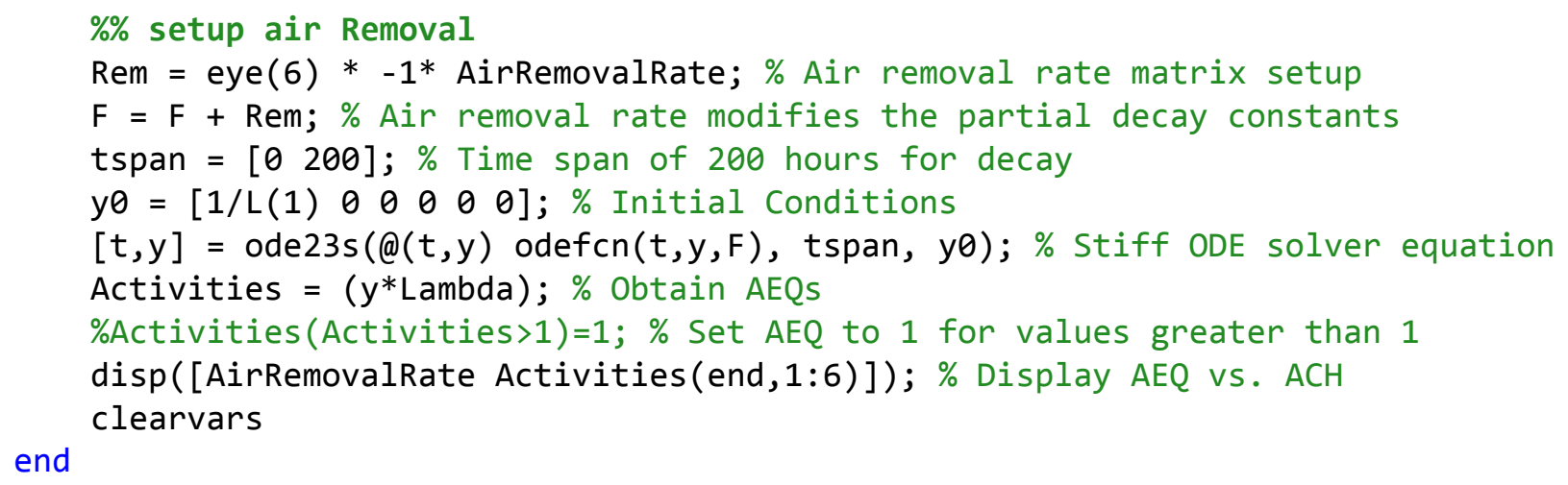




\section{Thoron Activity Equilibrium Factor Code}

$\%$ Thoron AEQ calculator

for AirRemovalRate $=\left[\begin{array}{llllllllllll}0 & 0.1 & 0.18 & 0.2 & 0.3 & 0.4 & 0.5 & 0.6 & 0.7 & 0.8 & 0.9 & 1\end{array}\right.$

$\begin{array}{lllllllllllllllll}1.1 & 1.2 & 1.3 & 1.4 & 1.5 & 1.6 & 1.7 & 1.8 & 1.9 & 2 & 2.1 & 2.2 & 2.3 & 2.4 & 2.5 & 2.6 & 2.7\end{array}$

$\begin{array}{lllllllllllllllllll}2.8 & 2.9 & 3 & 3.1 & 3.2 & 3.3 & 3.4 & 3.5 & 3.6 & 3.7 & 3.8 & 3.9 & 4 & 5 & 6 & 7 & 8 & 9 & 10\end{array}$

$\begin{array}{llllllllllllllllllll}15 & 20 & 25 & 30 & 35 & 40 & 45 & 50 & 55 & 60 & 65 & 70 & 75 & 80 & 85 & 90 & 95 & 100 & 200 & 300\end{array}$

$\left.\begin{array}{llllll}400 & 500 & 600 & 700 & 800 & 900\end{array}\right]$

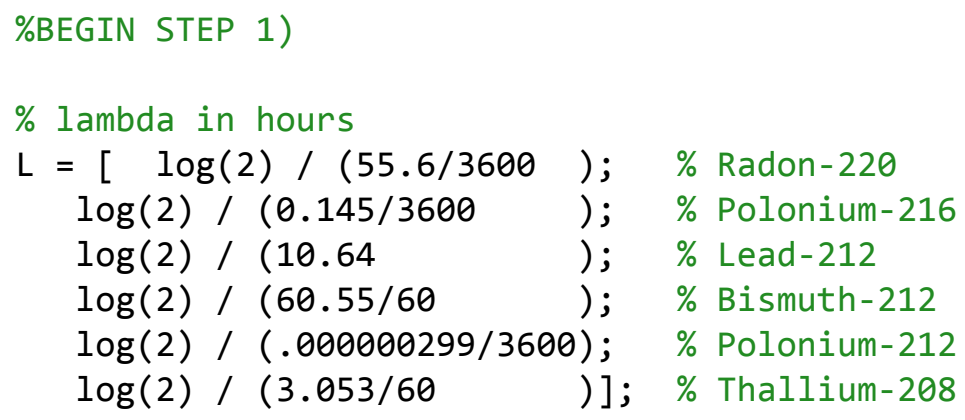




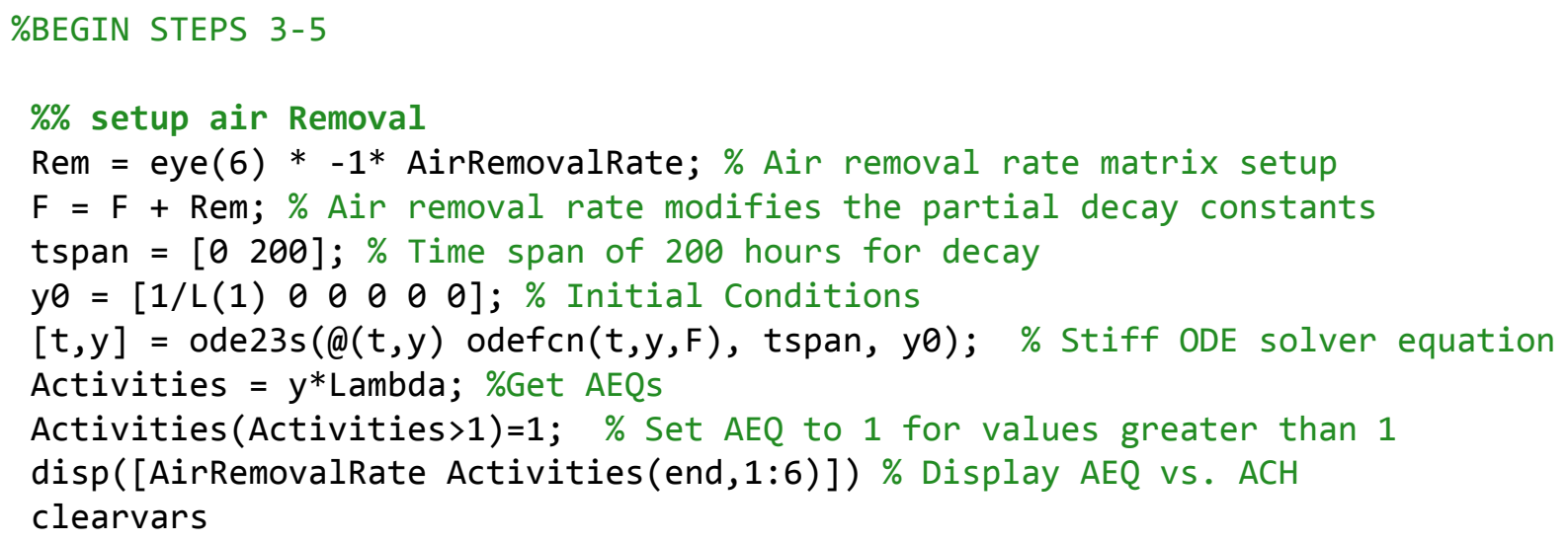

end 
$\underline{\text { Radon Activity Equilibrium Factor Code: }}$

$\%$ Radon AEQ calculator

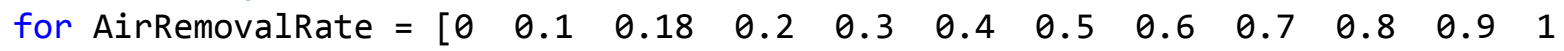

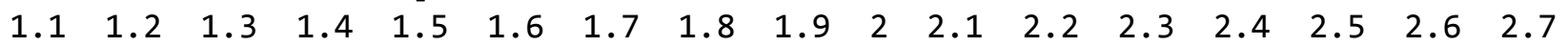

$\begin{array}{lllllllllllllllllll}2.8 & 2.9 & 3 & 3.1 & 3.2 & 3.3 & 3.4 & 3.5 & 3.6 & 3.7 & 3.8 & 3.9 & 4 & 5 & 6 & 7 & 8 & 9 & 10\end{array}$

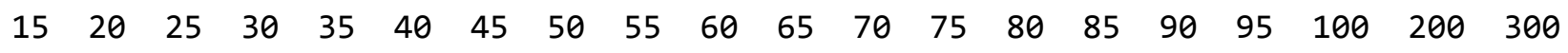

$\left.\begin{array}{llllll}400 & 500 & 600 & 700 & 800 & 900\end{array}\right]$

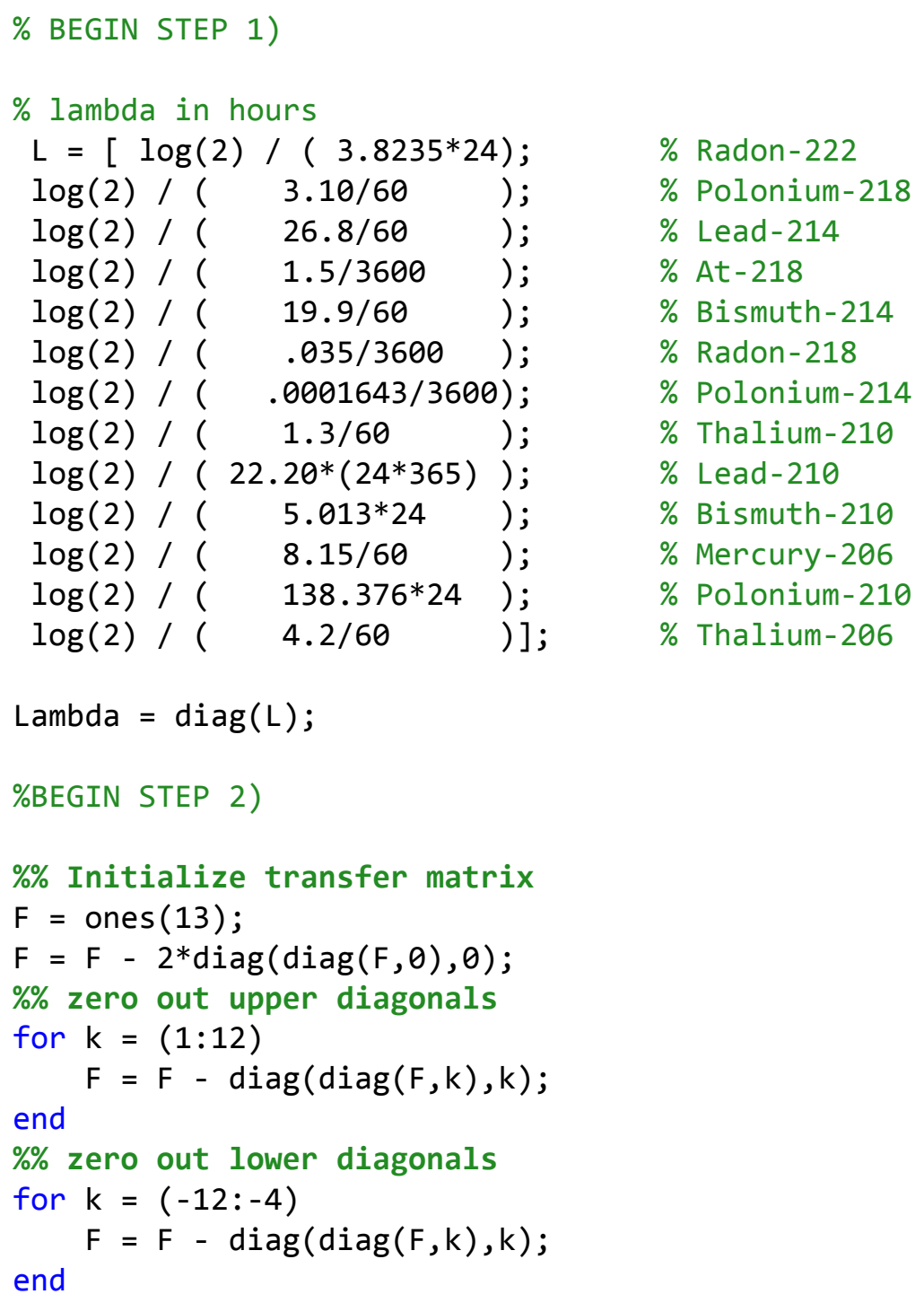




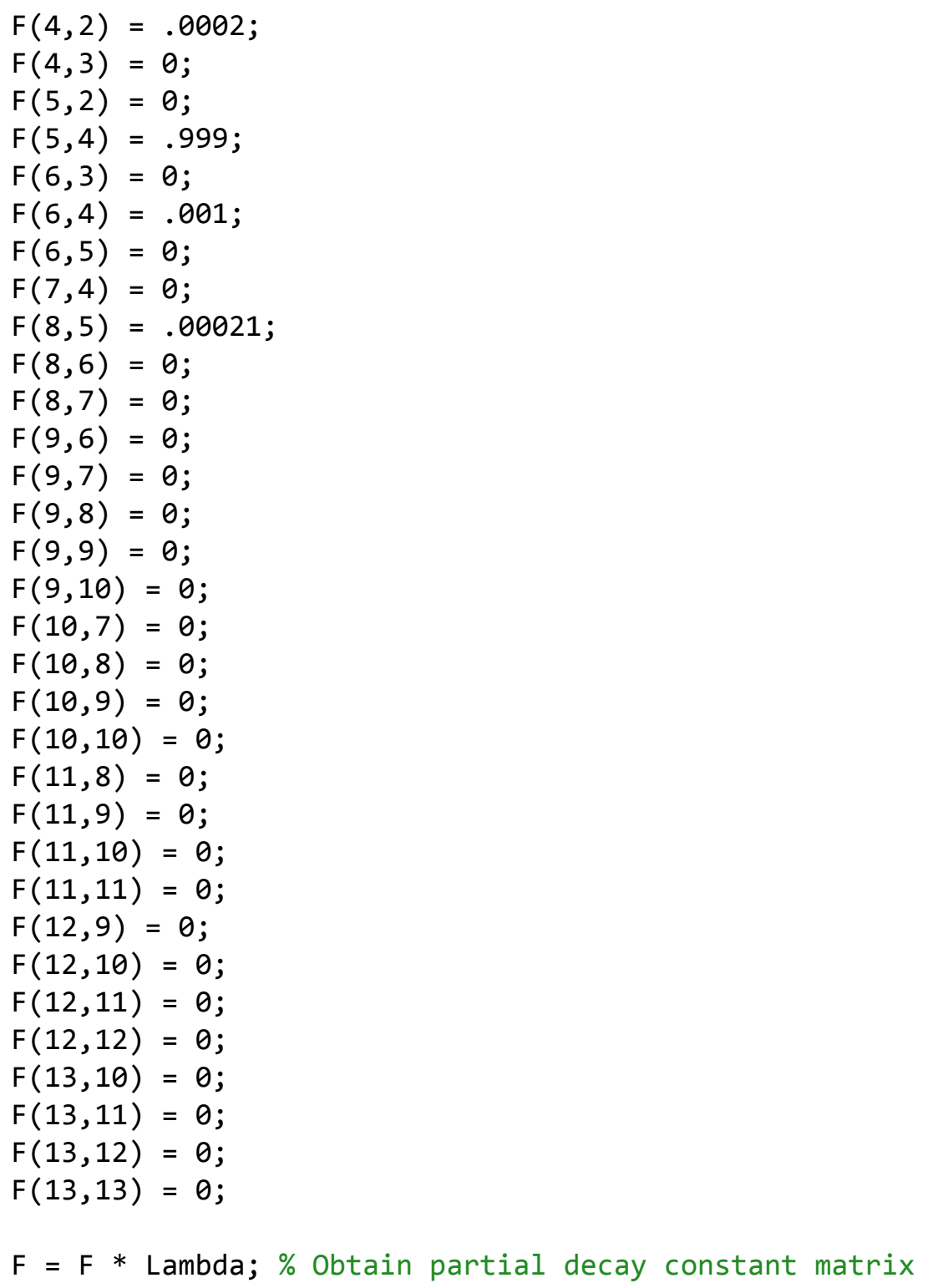

\%BEGIN STEPS 3-5

$\%$ setup air Removal

Rem $=$ eye $(13) *-1 *$ AirRemovalRate; \% Air removal rate matrix setup $\mathrm{F}=\mathrm{F}+\mathrm{Rem} ; \%$ Air removal rate modifies the partial decay constants tspan $=\left[\begin{array}{ll}\theta & 200\end{array}\right]$; $\%$ Time span of 200 hours for decay y $0=[1 / L(1) \quad 0 \quad 0 \quad 0 \quad 00000000000] ; \%$ Initial Conditions

$[t, y]=\operatorname{ode23s}(@(t, y) \operatorname{odefcn}(t, y, F)$, tspan, y0); \% Stiff ODE solver equation Activities $=\mathrm{y} *$ Lambda; $\%$ Obtain AEQs

Activities(Activities $>1$ ) $=1$; \% Set $A E Q$ to 1 for values greater than 1 disp([AirRemovalRate Activities(end,1:13)]) \% Display AEQ vs. ACH clearvars

end 
\%\% First-order ODE function to solve for number of atoms undergoing alpha/beta decay

function dydt $=\operatorname{odefcn}(\sim, y, F)$

$\mathrm{dydt}=\mathrm{F}^{*} \mathrm{y}$;

$\operatorname{dydt}(1)=0$;

end 

APPENDIX E. ACTIVITY EQUILIBRIUM FACTOR CALCULATION SUPPLEMENTAL GUIDE 

Variables from MATLAB are referenced in quotations below. Each step of the $\mathrm{A}_{\mathrm{eq}}$ calculation method is referenced for radon, thoron, and actinon by comments in the supporting MATLAB files in Appendix D. This step-by-step methodology is identical for radon, thoron, and actinon $\mathrm{A}_{\mathrm{eq}}$ calculations. An additional derivation that shows the relationship between air exchange rate and $\mathrm{A}_{\mathrm{eq}}$ is provided at the end of this appendix.

1) The "Lambda" diagonal matrix " $L$ " is set up. This matrix is comprised of the total decay constants for each radionuclide of the decay chain. The total decay constant is the probability that a radionuclide will undergo any type of decay, whether it be alpha decay, beta decay, etc.

2) The "F" matrix is set up. This matrix is comprised of the partial decay constants for each radionuclide of the decay chain. Consider the following equation:

$$
B F(\text { branching fraction })=\frac{\lambda_{i}}{\lambda}
$$

The branching fraction is a quantity that tells how often a radionuclide will undergo a specific decay mode. The partial decay constant, $\lambda_{i}$, represents the probability that a radionuclide will undergo a specific decay mode. The total decay constant, $\lambda$, is shown in the denominator. Once the " $F$ " matrix is initialized with corresponding branching fractions for each radionuclide, it is then multiplied by the "Lambda" matrix to obtain the new partial decay constant matrix " $\mathrm{F}$ ".

3) The air removal rate is denoted by "Rem". "Rem" is comprised of identity matrices for each of the air removal rates. Each of the air removal matrices are added to the "F" matrices. By adding the "F" matrices to the "Rem" matrices, the probabilities of undergoing a certain decay mode (partial decay constants) are modified to account for the air removal rates as shown in step 4.

4) A differential equation is set up to calculate the number of atoms undergoing alpha/beta decay for each radionuclide of the decay chain. This ODE (ordinary differential equation) is "stiff", since the step sizes taken by the solver are very small due to the half-lives of short-lived isotopes such as Po-212 in the thoron decay series. The very small step sizes make the "ode23" numerical solver in MATLAB unstable. Thus, "ode23s" is used to solve the stiff ODE. The equation is set up as follows:

$$
\left(\frac{d y}{d t}\right)=F * y
$$

$\mathrm{dy} / \mathrm{dt}=$ rate of change of number of atoms undergoing alpha/beta decay.

$\mathrm{F}\left(\mathrm{hr}^{-1}\right)=$ (matrices comprised of partial decay constants) + (air removal rate matrices).

$\mathrm{y} \quad=$ number of atoms undergoing alpha/beta decay.

This differential equation is separable, and when integrated, yields the following equation:

$$
y=e^{(F * t+C)}
$$


$\mathrm{Y}=$ number of atoms undergoing alpha/beta decay.

$\mathrm{F}\left(\mathrm{hr}^{-1}\right)=$ (partial decay constant matrices) + (air removal rate matrices).

$\mathrm{t}(\mathrm{hr}) \quad=$ amount of time at which decay occurs.

C = constant of integration.

Substituting the new " $F$ " $(F=F+r e m)$ into equation [11], yields:

$$
y=e^{((F+r e m) t+C)}
$$

The following example is used to prove the inverse relationship between the air removal rate and number of atoms, assuming a constant of integration equal to zero. Suppose "F" contains a partial decay constant of $(1 \text { hour })^{-1}$. Substituting $t=1$ hour at an air removal rate of $(2 \text { hour })^{-1}$ into equation [12] yields:

$$
y=e^{(-1-2) *(1)}=0.0498 \text { atoms }
$$

Note that the partial decay constant and air removal rate is negative. The partial decay constant is negative, since atoms decay at a negative exponential rate. The air removal rate is negative, since air is being subtracted from the total volume of air. Now, by increasing the air removal rate to $(5 \text { hours })^{-1}$ at $\mathrm{t}=1$ hour at $\mathrm{F}=(1 \text { hour })^{-1}$ :

$$
y=e^{(-1-5) * 1}=0.00248 \text { atoms }
$$

This proves that as the air exchange rate increases, the number of atoms undergoing beta/alpha decay decreases.

5) Once the number of atoms undergoing beta/alpha decay are found using the ODE solver over a period of two hundred hours at each air exchange rate, the values are multiplied by the lambda matrix as shown below from MATLAB to obtain the activities:

$$
\text { Activities }=y * \text { Lambda. }
$$

These activities are in units of concentration and are assumed to be in one liter of air. The parent radon radionuclides are always at a value of one picocurie per liter of air. Thus, the remaining progeny activities in the output from MATLAB are inherently in units of the $\mathrm{A}_{\text {eq }}$ (dimensionless), since they are all divided by a unit parent concentration of one picocurie per liter of air. Since the number of atoms decrease as the air removal rate increases, the $\mathrm{A}_{\mathrm{eq}}$ does as well. 